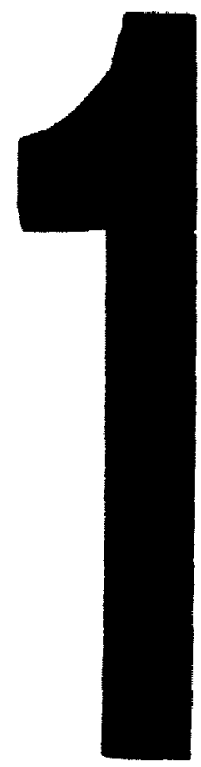

PM-1 31, "x 4 " PHOTOGRAPHIC MICROCOPY TARGET NBS 1010a ANSI/ISO $\# 2$ EQUIVALENT

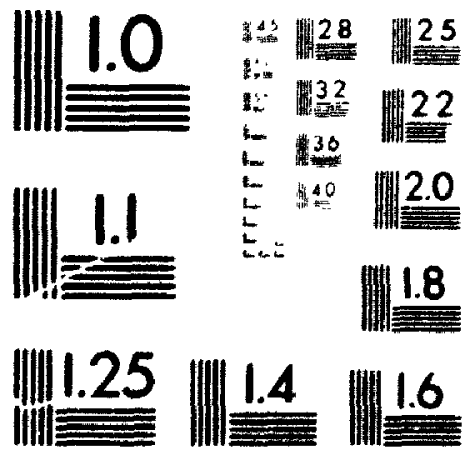


Acquisitions and

Biblographic Services Branch

395 Wellington Street

Ottawa. Ontario

KIA ON4
The quality of this .nicroform is heavily dependent upon the quality of the original thesis submitted for microfilming. Every effort has been made to ensure the highest quality of reproduction possible.

If pages are missing, contact the university which granted the degree.

Some pages may have indistinct print especially if the original pages were typed with a poor typewriter ribbon or if the university sent us an inferior photocopy.

Reproduction in full or in part of this microform is governed by the Canadian Copyright Act, R.S.C. 1970, c. C-30, and subsequent amendments.
La qualité de cette microforme dépend grandement de la qualité de la thèse soumise au microfilmage. Nous avons tout fait pour assurer une qualité supérieure de reproduction.

S'il manque des pages, veuillez communiquer avec l'université qui a conféré le grade.

La qualité d'impression de certaines pages peut laisser à désirer, surtout si les pages originales ont été dactylographiées à l'aide d'un ruban usé ou si l'université nous a fait parvenir une photocopie de qualité inférieure.

La reproduction, même partielle, de cette microforme est soumise à la Loi canadienne sur le droit d'auteur, SRC 1970, c. C-30, et ses amendements subséquents. 


\title{
Speculative Computation
}

\author{
for
}

\section{User Interfaces}

\author{
b y \\ David A. Scott
}

\begin{abstract}
A thesis submitted to the Faculty of Graduate Studies and Research

in partial fulfillment of the requirements for the degree of Master of Computer Science
\end{abstract}

School of Computer Science

Carleton University, Ottawa, Ontario

December 31, 1992

(C) Copyright 1992, David A. Scott 
National Library

of Canada

Acquisitions and

Bibliographic Services Branch

395 Wellington Street

Ottawa. Ontario

K1A ONA
Bibliotheque nationale

du Canada

Direction des acquisitions et

des services bibliographiques

395. ne Wellington

Ottawa (Ontario)

KIA ONA
The author has granted an irrevocable non-exclusive licence allowing the National Library of Canada to reproduce, loan, distribute or sell copies of his/her thesis by any means and in any form or format, making this thesis available to interested pirsons.
L'auteur a accordé une licence irrévocable et non exclusive permettant à la Bibliothèque nationale du Canada de reproduire, prêter, distribuer ou vendie des copies de sa thèse de quelque manière et sous quelque forme que ce soit pour mettre des exemplaires de cette thèse à la disposition des personnes intéressées.

L'auteur conserve la propriété du droit d'auteur qui protège sa thèse. Ni la thèse ni des extraits substantiels de celle-ci ne doivent être imprimés ou autrement reproduits sans son autorisation.

ISBN $\quad 0-315-84091-9$ 
The undersigned recommend to the Faculty of Graduate Studies and Research acceptance of the thesis

\section{Speculative Computation}

For

\section{User Interfaces}

submitted by David A. Scott

in partial fulfillment of the requirements for the degree of Master of Computer Science
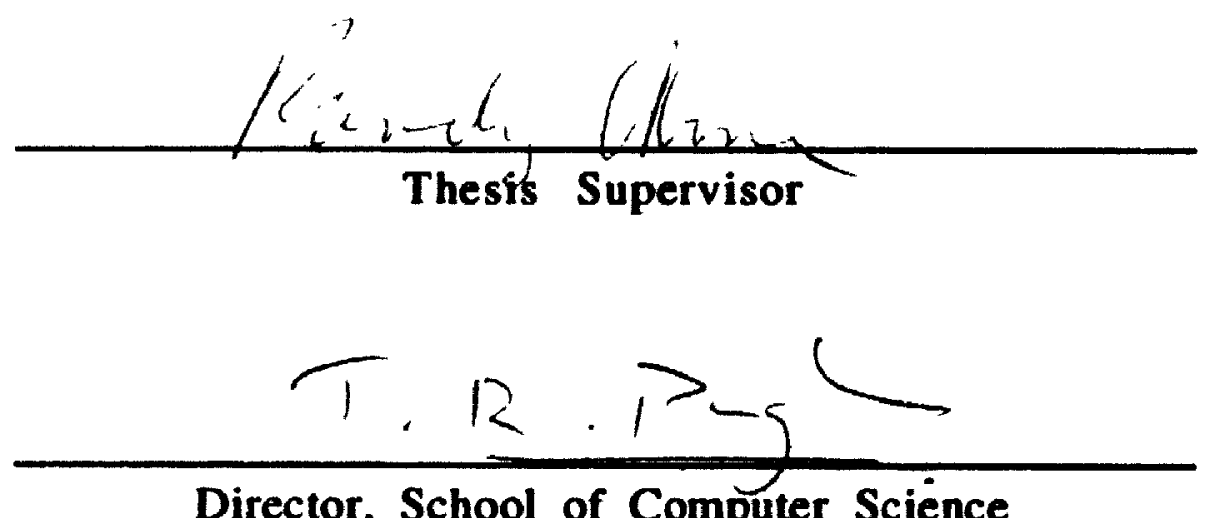

Director, School of Computer Science

Carleton University 


\begin{abstract}
In this thesis we describe the use of Osborne's model of speculative computation [Osborne] to improve the response time of an interactive application to a user's requests. We present a technique for modeling the dialog between the user and the application to determine what portions of the dialog can best benefit from speculative compulation. We present techniques for analyzing the model of a dialog to determint how to allocate processing resources so that the expected overall response time is minimized. In order to improve the effectiveness of using speculative computation, we propose an extension to Osborne's sponsor model for speculative computation to include a duration attribute. We determine the effectiveness of speculative computation at reducing response time by performing experiments to compare the success of speculative computation versus sequential and conventional mandatory parallelism approaches.
\end{abstract}




\section{ACKNOWLEDGMENTS}

1 would like to thank the following people for their input and support:

Randy Osborne, my thesis supervisor, whose patience and support have been essential to the completion of thesis, and for reading more drafts of this thesis than any person should be forced to.

Sheila A. Krawchuk. my better half, for proving just how much better she really is.

Mike Keith, for being there when I needed someone to talk to.

Everyone in main office for making my stay here an enjoyable and entertaining experience.

Suki, Skii, Storm and Sheba, for always hiding my pens when I needed them most. And Sunny for making me see the "light" at the end of the tunnel. 


\section{TABLE OF CONTENTS}

Chapter 1 - Introduction ............................................................. 1

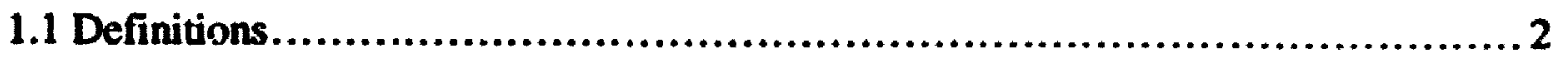

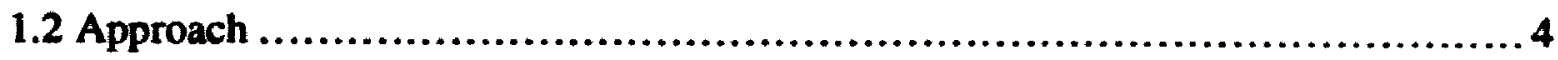

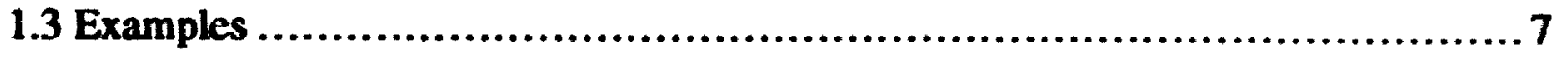

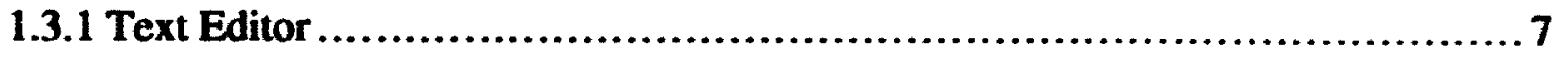

1.3.2 Dungeon Explorer Game.................................................9

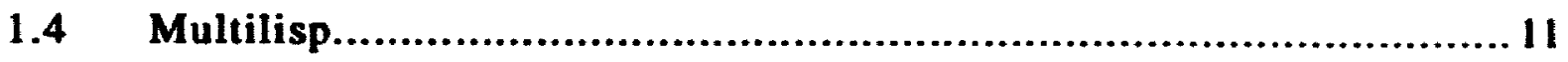

1.5 Speculative Computation............................................................ 11

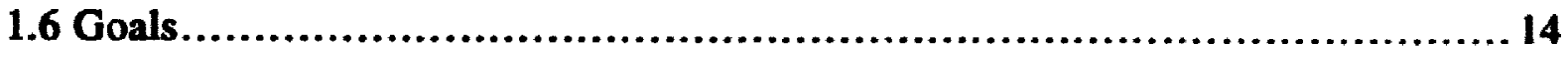

1.7 Preview ......................................................................... 14

Chapter 2 - Related Work ....................................................... 15

2.1 Applications ................................................................ 15

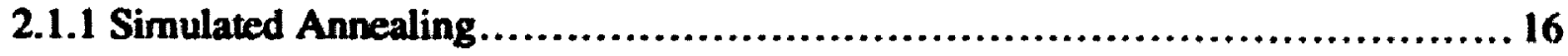

2.1.2 Optimistic Make ........................................................... 17

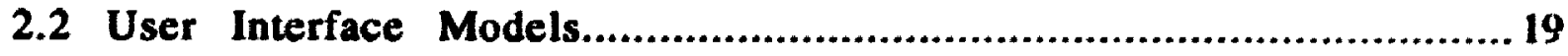

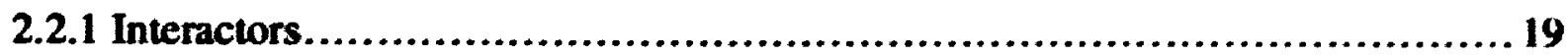

2.2.2 User Interface Modelling using State Machines .............................20

2.2.3 Properties of a Model for Parallel Computation .............................21

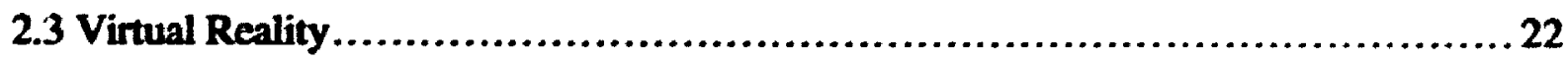

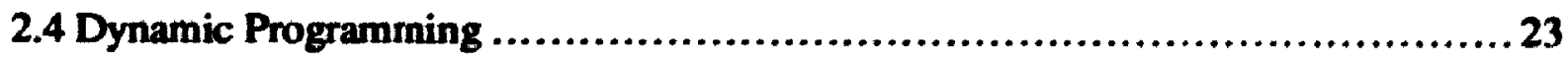

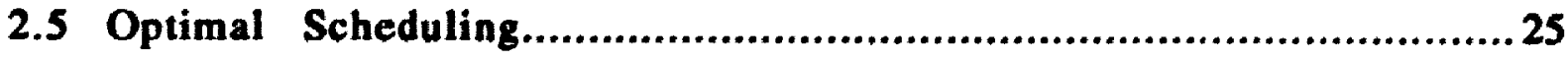

Chapter 3 - A Model For User Interface Analysis...............................27

3.1 Graph Model Of Dungeon Exploner Game ................................. 28 


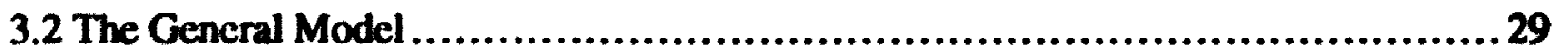

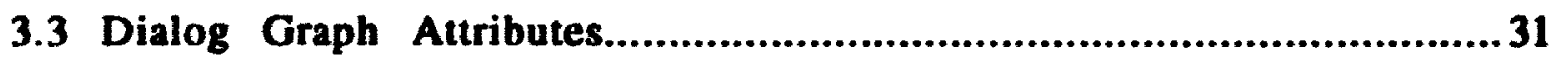

3.5 Dialog Graph Classifications................................................. 33

3.6 Dialog Graph Reductions............................................................ 37

3.6.1 Splitting Multiple Nodes ...................................................... 38

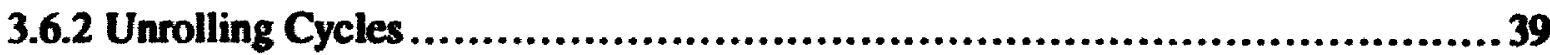

3.7 Constructing a Model .......................................................42

3.7.1 Basic Steps............................................................... 42

3.7.2 Examples of Model Construction.....................................................43

3.7.2.1 Model for the Simple Text Editor .............................................43

3.3.2.2 Model for the Dungeon Exploration Game ..................................46

3.8 Characteristics of Speculative Interfaces ........................................ 48

3.8.1 Characteristics ................................................................ 48

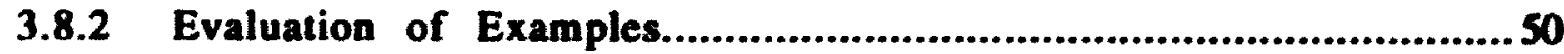

3.8.2.1 Evaluation of the Simple Text Editor .......................................5 50

3.8.2.2 Evaluation of the Dungeon Exploration Game ..............................51

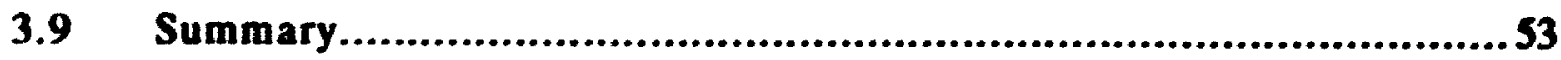

Chapter 4 - Calculating Response Time ............................................54

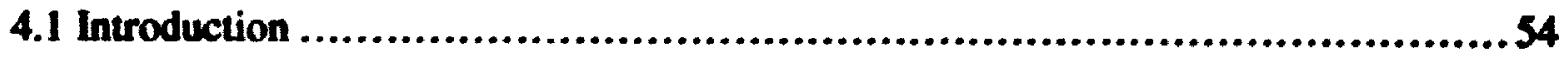

4.2 Request Groups...............................................................5 57

4.3 Expected Response Time ....................................................58

4.4 Calculating the Overall Expected Response Time.......................................59

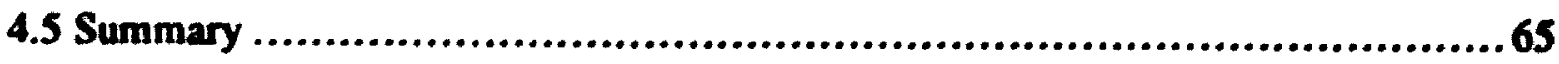

Chapter 5 - Constructing Request Groups ........................................66

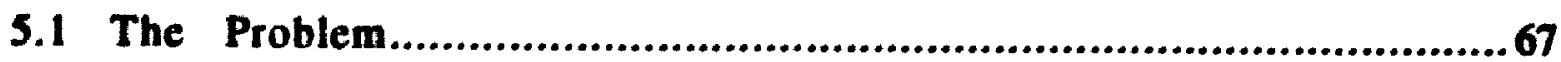




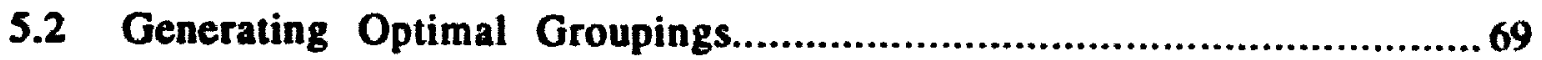

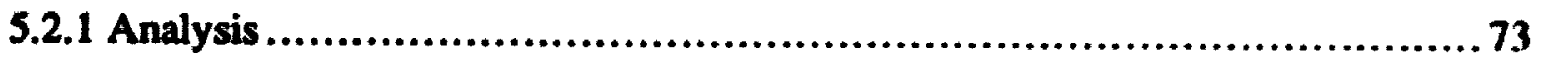

5.2.2 Deriving Heuristics .......................................................... 84

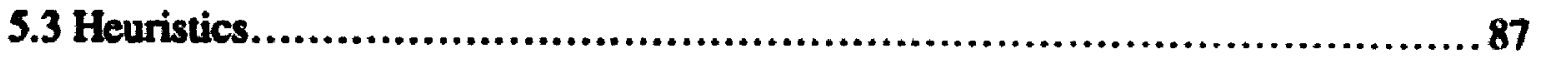

5.3.1 Heuristic 1 - Select Most Probable Nodes ......................................87

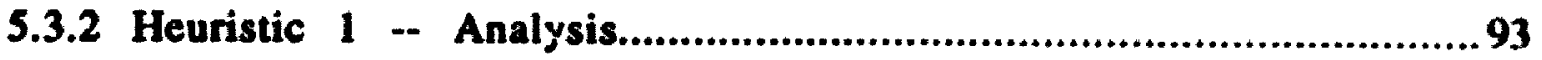

5.3.3 Heuristic 2 -- Most Probable Path........................................................97

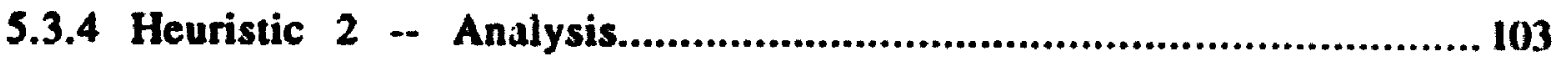

5.4 Non-Constant Compute and Think Times ....................................... 108

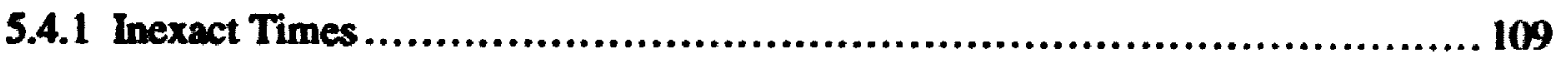

5.4.2 Unknown Times................................................................112

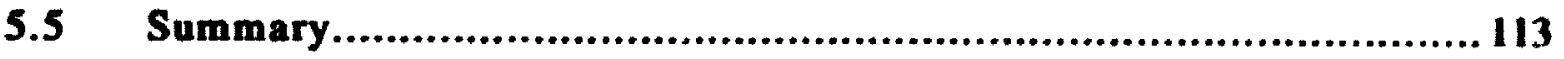

Chapter 6 - Group Scheduling................................................. 114

6.1 Introduction .................................................................... 114

6.2 Requirements of Group Scheduling............................................115

6.3 Current Support for Group Scheduling in Speculative Computation.................... 118

6.4 Implementing Group Scheduling ................................................ 119

6.5 Proposed Sponsor Model Extension for Known Times ......................... 122

6.5.1 Adding Duration Attribute................................................ 124

6.5.2 Group Scheduling Using Duration Attribute........................................ 129

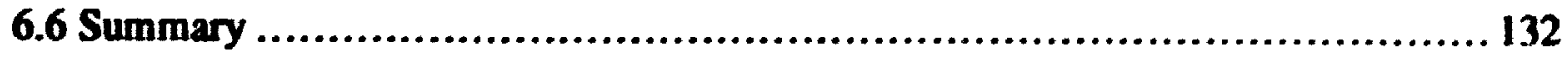

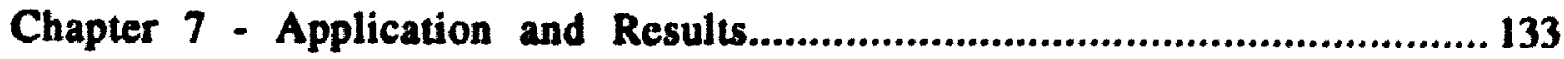

7.1 The Application ............................................................. 133

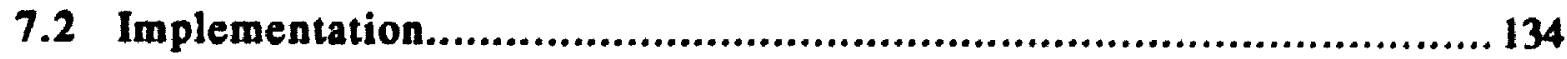




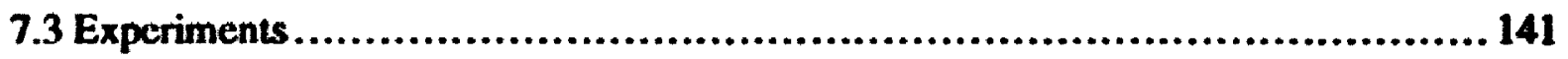

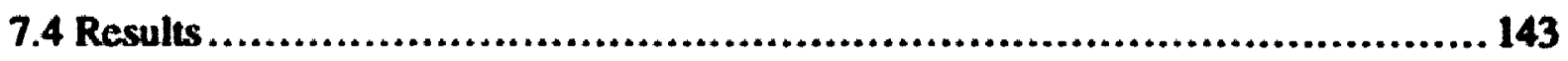

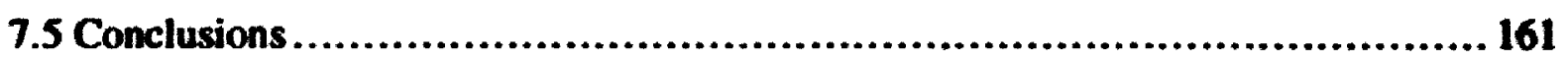

Chapter 8 - Conclusions and Future Work........................................... 163

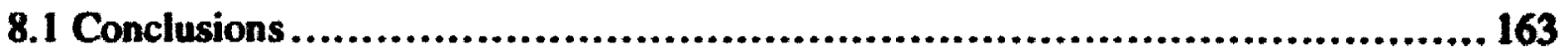

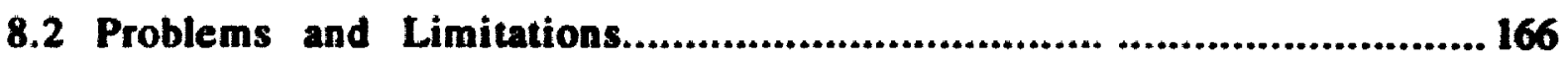

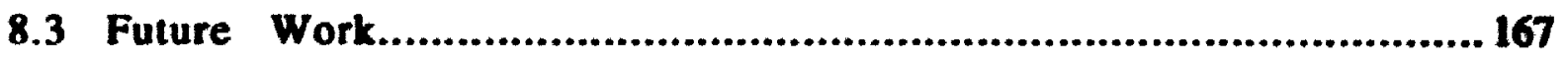

8.3.1 Theory of User/Interface Dialogs ................................................. 167

8.3.2 User Interface Applications ................................................ 168

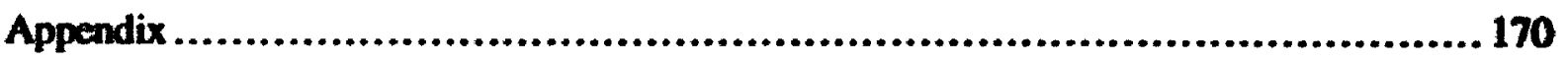

Algorithm Computing the E[ORT] of a Dialog Graph................................ 170

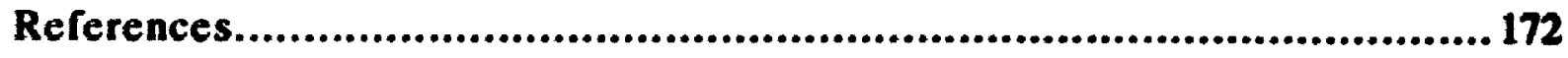




\section{LIST OF FIGURES}

Figure 1.0 - User/Interface Dialog ............................................... 3

Figure 1.1 - Think and Compute Time Relationships........................................5

Figure 1.2 - The Effect of "Eager" Computation on Response Time ..................8

Figure 1.3 - Simple Text Editor Menu Hieranchy .................................8

Figure 3.0 - Dungeon Explorer Game User/Interface Dialog Model.................... 29

Figure 3.1 - Symbols Use in Constructing a User/Interface Dialog Model .............. 30

Figure 3.2 - User-Application Dialog Loop.......................................31

Figure 3.3 - Movement of Focus With New Requests .............................. 31

Figure 3.4 - Dependant Versus Independant Paths..............................................35

Figure 3.5 - Dependance Versus Independance of Multiple Visits...................... 36

Figure 3.6 - Removing Multiple Paths Using Node Splitting................................. 38

Figure 3.7 Representing Internode Dependencies Introduced by Node Splitting........... 39

Figure 3.8 - Unrolling a Degenerative Cycle to Form an Acyclic Tree .................41

Figure 3.9 - User/Interface Dialog Model of the Simple Text Editor.........................46

Figure 4.0 - Graph of a Simple User/Interface Dialog.................................55

Figure 4.1 - Computation of Total Processing Time Available ........................56

Figure 4.2 - Graph of a Simple User/Interface Dialog..............................63

Figure 4.3 - Graph of a Simple User/Interface Dialog..............................64

Figure 5.0 - Simple User/Interface Dialog .......................................69

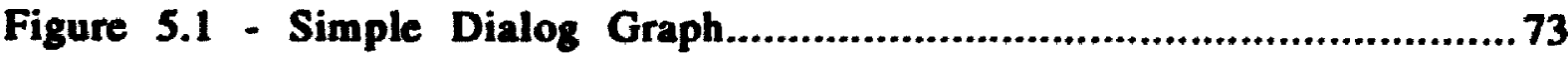

Figure 5.2 - Possible Request Groups For Dialog Graph...........................77

Figure 5.3 - Optimal Groupings for a Complete Depth 4 Binary Tree.................80

Figure 5.4 - Non-Optimal Partial Grouping for Depth 4 Complete Binary Tree............ 81

Figure 5.5 - Groups for a Depth 3 Complete Binary Tree..................................82 
Figure 5.6 - Possible Groupings for a Depth 3 Complete Binary Tree ..................83

Figure 5.7 - Partial Groupings ..................................................84

Figure 5.8 - Descrepncies Between RGC Algorithm and Select Most Probable ..........89

Figure 5.9 - Depth Sensitivity Example using 3-ary Troe ..................................95

Figure 5.10 - K-ary Tree with ith Arc Having Same Probability........................ 104

Figure 5.11 - Recurrence Relation for Select Most Probable Path Heuristic.............. 105

Figure 5.12 - Compute Times as Discrete Distributions................................. 109

Figure 5.13 - Expression of Variable Compute Times.. ....................................... 110

Figure 5.14 - Integration of Variable Compute Times ....................................111

Figure 6.0 - Node Operations During the Scheduling Process .......................... 117

Figure 6.1 - Implementation of Group Scheduling for Unknown Times.................... 121

Figure 6.2 - Task/Engine Components for Support of Duration Atribute ................ 128

Figure 6.3 - Implementation of Group Scheduling for Known Times....................... 131

Figure 7.0 - Multilisp+SC Code for Reading Maze Parameters ........................ 138

Figure 7.1 - Messages Accepttd by Room Objects .................................... 139

Figure 7.2 - Multilisp+SC Code for Dungeon Exploration Game............................ 141

Figure 7.3 - Dialog Graph Structure .............................................. 153 


\section{LIST OF TABLES}

Table 5.0 - Results of Select Most Probable Nodes Heuristic .........................90

Table 5.1 - Results of Select Most Probable Path Heuristic................................... 102

Table 7.1 - Constant Compute Times of 750 ..................................... 145

Table 7.2 - Random Compute Times Between 500 and 1000 ........................ 148

Table 7.3 - Random Compute Times Between 0 and 1500 .......................... 150

Table 7.4 - Random Compule Times Between 500 and 1000 and Random Arc ........ 152

Table 7.5 - Large Variation between Room Compute Times................................. 154

Table 7.6 - Compute Times of 750 and Think Times of 250 ..................... 156

Table 7.7 - Comparison of RGC Algorithm and Select Most Probable Nodes .......... 158

Table 7.8 - Comparison of RGC Algorithm and Select Most Probable Nodes .......... 160

Table 7.9 Summary of Results of Experiments............................................ 161 


\section{Chapter 1}

\section{Introduction}

When an interactive dialog exists between a user and a computer application, there are periods of time when the user pauses in his/her interaction with the application. These periods occur when the user is examining the result of some task that the application has performed, or when an external stimulus, for example a telephone, distracts the user. During these idle periods the application could be performing computations in an effort to anticipate some of the user's future requests. A reduction in the time required to respond to a user's request may be possible if the application is accurate in its predictions.

There are two main reasons why it can be difficult to accurately predict what requests a user may make of an application. First, users are human and a human does not always make rational decisions, nor do two humans think exactly alike. Secondly, the "user" of the system may be some other application whose requests might be unpredictable (perhaps a human user is making request of "that" system). 
Speculative computation [Osborne] is a type of computation by which the application program may guess which computations a user may requine next. With speculative computation, we perform "eager" evaluation of computations. This may result in faster execution (and possibly extraneous computation).

This thesis is an investigation into the use of speculative coisputation to exploit the idle periods in a user/interface dialog. Our goal in this thesis is to show that speculative computation techniques can be used to decrease user/interface response times. We do not attempt to find exact solutions to the problem of scheduling tasks so that the response times are minimized. Instead we concentrate on the development of efficient heuristics and how to integrate speculative computation into the activities performed within a user interface. We present results from experiments we conducted to measure the success of speculative computation at minimizing reponse time.

The remainder of this chapter is divided into sections that give a definition of user/interface dialog and other terms used in this thesis (Section 1.1), describe the approach taken towards exploiting the idle periods (Section 1.2), present some examples of user/interface dialog (Section 1.3), briefly discuss the Multilisp language (Section 1.4), discuss speculative computation (Section 1.5), describe our goals (Section 1.6), and present an overview of the remainder of this thesis (Section 1.7).

\subsection{Definitions}

Figure 1.0 illustrates the user/interface dialog at its most primitive level. The user communicates his/her needs to the application by making requests. In turn the application 
accepts the requests made by the user and performs the computations associated with the request, returning the result of the computation to the user.

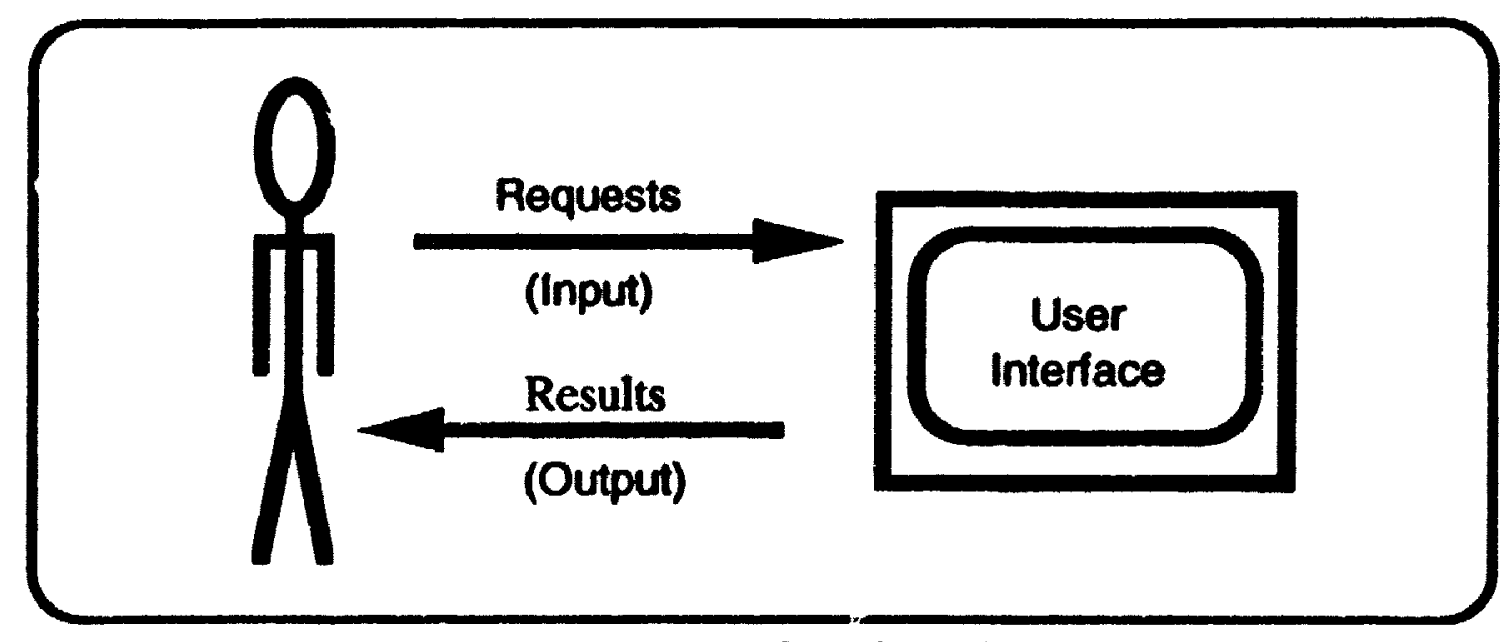

Figure 1.0 -. User/Interface Dialog

Our main concern is with the consumption of time, both wall-clock and processor, during the user/interface dialog. To this end, we define three categories of time:

\section{Compute Time}

Upon receipt of a request, this is the amount of time required by the application to compute the response on a single processor.

\section{Think Time}

The amount of time that elapses from the receipt of a response to the last request until the user makes the next request.

\section{Response Time}

The amount of time that the user has to wait for the application to return a result to his/her request, once the request has been made. Response time can be shorter than 
Compute Time if we can perform some of the computations associated with a request before the user issues the request. For example if a request takes 5 units of time to compute and 2 time units of computing are done before the user issues the request, the reponse time for the request is 3 time units.

\subsection{Approach}

There are three means of improving the response time for an application: reduce the compute time for the requests or overlap the computation of the various requests. The use of optimizing compilers, efficient coding practices, and the intelligent exploitation of parallelism can shorten the compute time for requests. We have chosen to pursue the investigation of overlapping the computation using speculative computation tochniques to achieve the overlap.

There are 2 possible scenarios for overlapping think and compute times for requests which are made in sequence, as illustrated in Figure 1.1. The main difference between scenarios $A$ and $B$ is that we start think time " $\mathrm{i}$ " early, speculating on the result of request " $\mathrm{i}-1$ ". With scenario A, we have overlapped the think and compute times of different requests. With this scenario we are allowing the user to think about what requests he/she would like to make next, even before he/she knows the result of the current request. For example, the user may have just requested to have an image displayed on the screen. As we are displaying the image on the screen, we tell the user what operation he/she can perform on the image once it has been completely displayed (e.g., enlarge, rotate). This gives the user the opportunity to consider what his/her next request will be before the current request has been completely processed. 


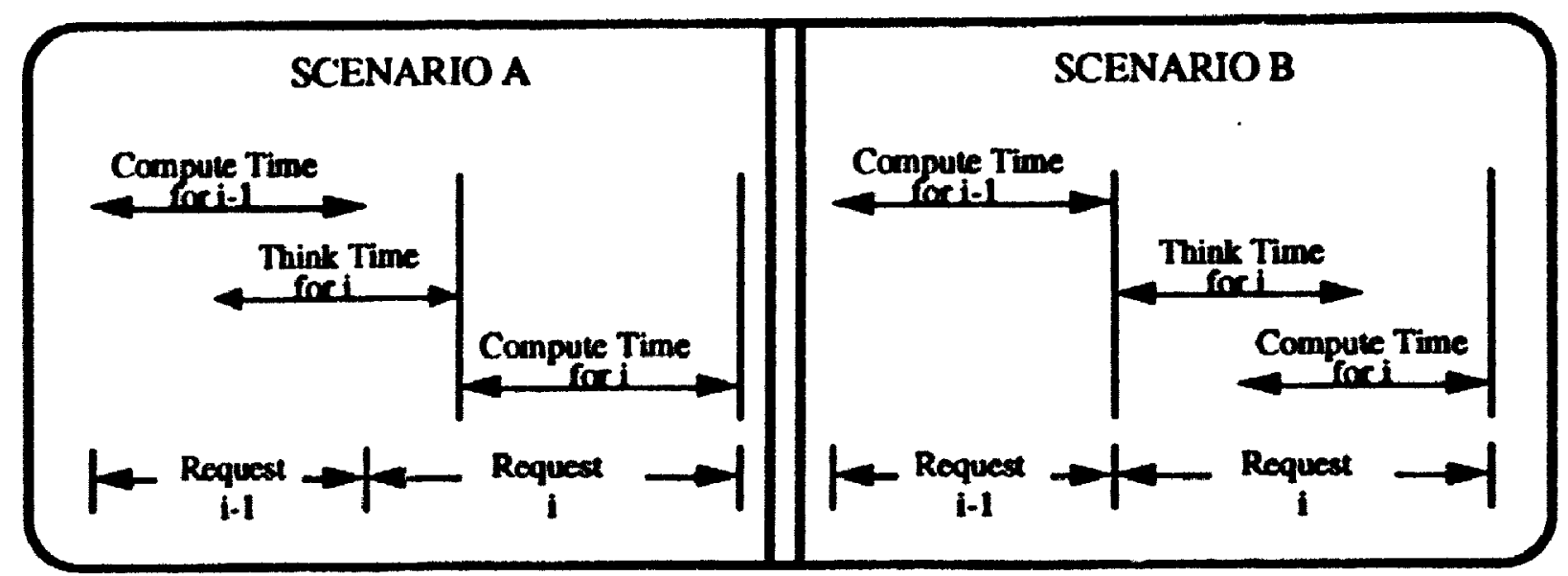

Figure 1.1 - Think and Compute Time Relationships

With scenario B, the user is not given the opportunity to think about what his/her request will be until the results of his/her current request have been computed. With this scenario, we are only using speculation to achieve response time improvements. In this thesis, we will only be considering scenario B. However, scenario A still has good potential for improving response time, especially when the computation associated with a request requires a long time to produce a complete result. If the user can consider decisions for future requests based on the parial results of the current request, then the response time can be decreased by allowing the user to do so. We can model scenario $A$ using scenario $B$ by redefining the think time as time from result of request "i-1". 


\section{DIAGRAM A - NO SPECULATION OF REQUESTS}

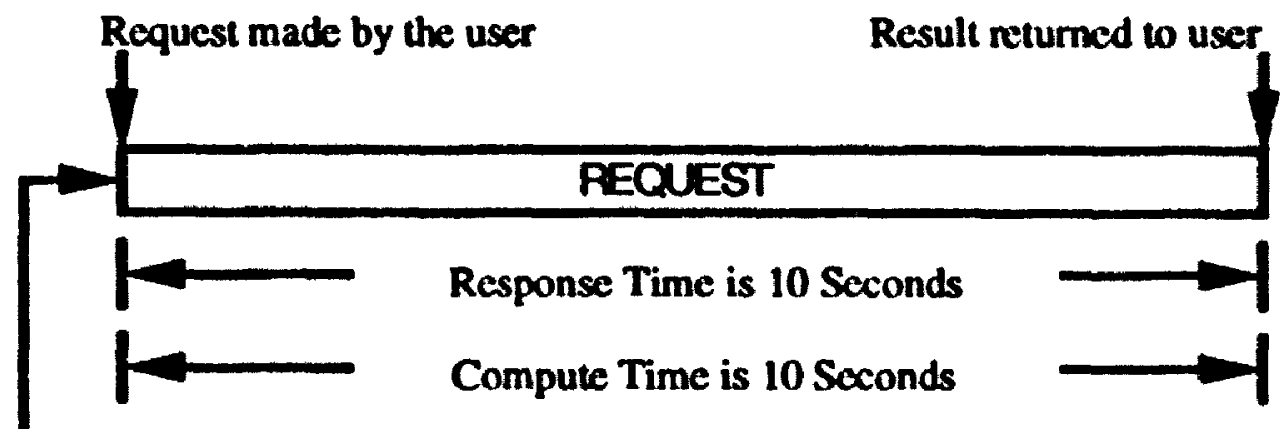

User Request that takes 10 seconds to process

\section{DIAGRAM B - SPECULATION OF REQUESTS}

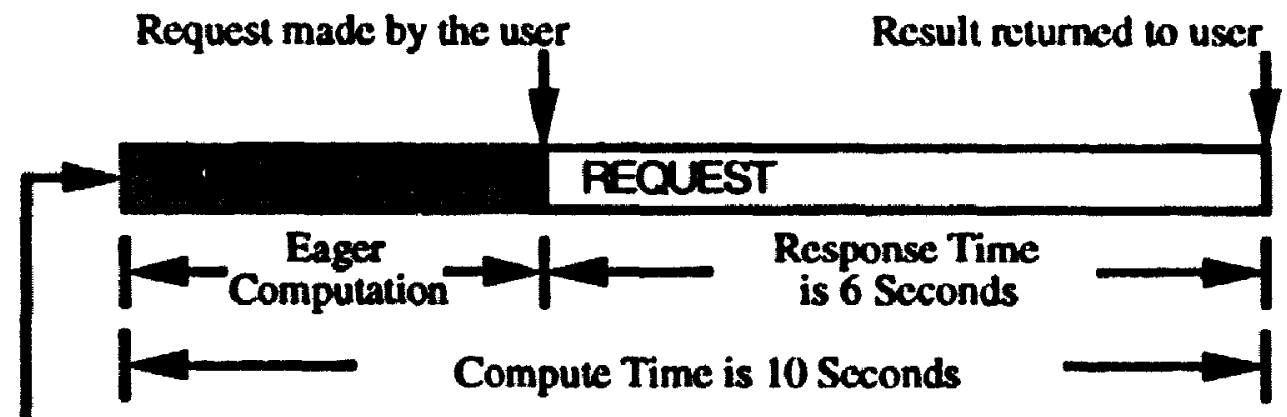

User Request that takes 10 seconds to process

Figure 1.2 - Effect of "Eager" Computation on Response Time

If we employ no form of speculative computation, the response time for a user request is the same as the compute time for the request. However, by using speculative computing, if we correctly guess the user's next request we will realize a reduction in the response time. The reduction in time will be equal to the amount of the computation performed before the receipt of the request. If we guess wrong and fail to perform any "eager" computation on

\footnotetext{
-We define "eager" computation as the computation that is done before we actually know that we will require the result of the computaion.
} 
the user's request, the application has only consumed some excess resources that would have boen idle and a marginal increase may occur in the response time for a user's request, due to the overhead of possibly halting the speculative computations undertaken during the idle period. Figure 1.2 illustrates the impact of speculative computation on response time.

To reduce the response time for an application by overlapping requests, we need some means to determine which requests should be overiapped. We propose a model for identifying requests that should be overlapped. We also propose a method to overlap the execution of these requests using speculative computation. We implement an example application which uses the proposed procedure. We compare the speedup results from this application to the results of a version of the application that uses no overlap and to another version that uses mandatory parallelism to implement the overlap of requests.

\subsection{Examples}

We present two quite different examples of user/interface dialogs. We use these examples to motivate discussion in the remainder of the thesis. These two dialogs represent contrasting types of user/interface dialogs. In Chapter 3, we introduce a model for the analysis of these dialogs to determine if it is worth while to program them to exploit the use of speculative computing.

\subsubsection{Text Editor}

Figure 1.3 depicts the functions that a simple text editor might present to a user. For instance, the user can enter text, format text already entered, and save or open files. This type of dialog is typical of many applications that currently exist (e.g., spreadsheets, word 
processors). The rigid definition of the functions available to the user limits the variety of user requests. In contrast, the exact detail of the input to these functions is not rigid. For instance, we can see from Figure 1.3, under the File heading, that the user may either save. open or quit editing a file. This implies that the user may not move or rename the file. However, the editor does not impose a naming convention for the file.

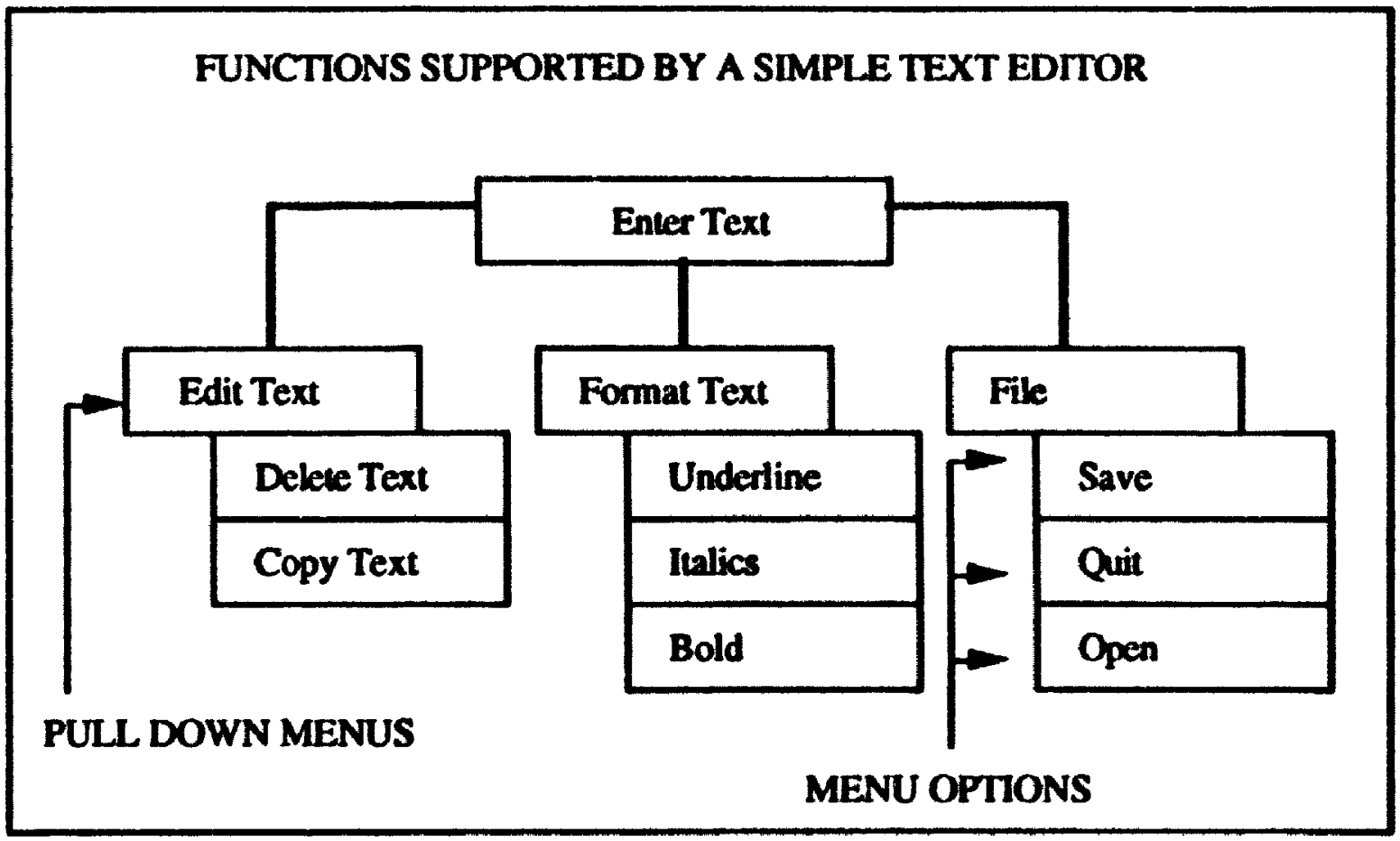

Figure 1.3 - Menu Hierarchy and Functions Supported by a Simple Text Editor

A typical dialog that the user might carry on with the application would start with the user opening a file using the Open option. Once the file is open, the user would enter text. perhaps underlining some key points by using the Underline option. Once the user feels that the modifications he/she has made to the file are correct, he/she saves the file using the Save option and then exits the dialog using the Quit option. 
In an effort to reduce response time, we could attempt to predict the various operations that the user would perform during the course of a dialog. For instance, when the user enters into a dialog with the application, we can be quite certain that the first request the user will make is to open a file. We could attempt to guess which file the user wculd request. Perhaps during the initialization of the application, several recently used files could be loaded into a cache. In Chapter 3, we will consider in more detail, what the possibilities are for improving the response time for applications by using speculative computation.

\subsubsection{Dungeon Explorer Game}

Figure 1.4 depicts the functions that a user may perform in a dungeon exploration game. In this game, the dialog between the user and the application is highly interactive: the user can move freely about the room, picking up objects and exploring. The user would use some advanced input device, a dataglove for example, 10 communicate his/her requests to the application. There are also very few constraints placed on what requests the user may make of the application. For instance, in the dungeon explorer game, the only constraints placed on the user are that he/she can not walk through a wall or see what is behind a door without opening it first

The freedom of movement allowed in the Dungeon Explorer game is indicative of a new style of user interface called "Virtual Reality". With a Virtual Reality application the user is, for the most pan, frec to tailor and explore his/her environment as he/she sees fit. We feel that the development of more applications of this style will be facilitated by the higher availability of parallel computers in the future and expect it to be one of the styles of user interface that can benefit from the use of speculative computation. 


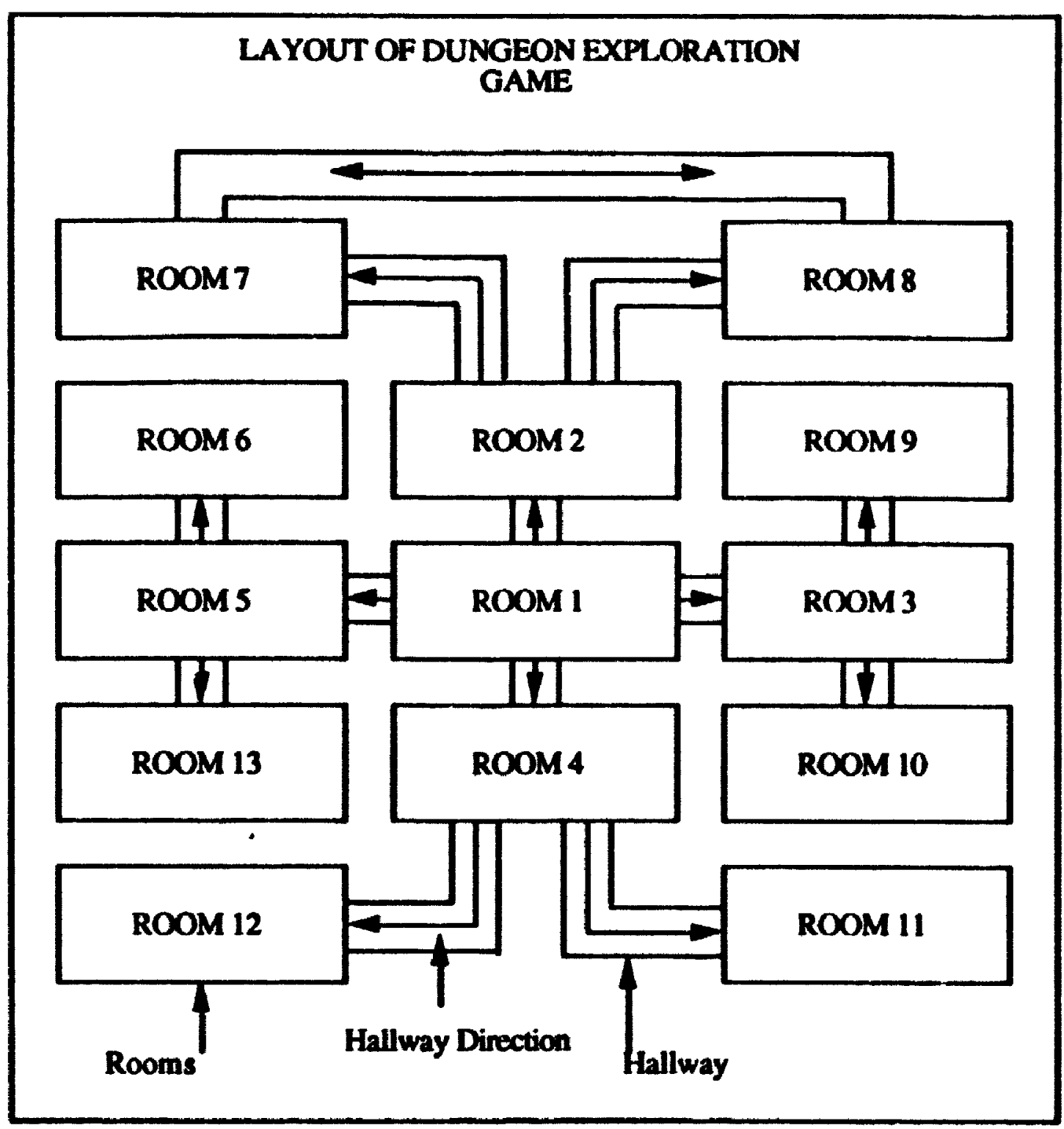

Figure 1.4 - Structure of a Dungeons \& Dragons Style Adventure Game

For the purposes of discussion, we will consider this example in a restricted format, where the user may only request to move from room to room. This will not compromise the integrity of the example since any transformations we apply to the restricted version are still applicable to the unrestricted version. 


\subsection{Multilisp}

We have chosen to use a model of speculative computation as defined in [Osborne]. The implementation of the model is an extension of Multilisp [Halstead]. Multilisp is a dialect of the Scheme [Dybvig] programming language with explicit parallelism constructs, includes side-effects and is based on a shared memory paradigm. The Multilisp language facilitates the fast prototyping of applications and provides solid support for Artificial Intelligence applications. Multilisp provides an explicit parallelism construct called future; (future $\exp d o c$ ) - creates a task to cvaluate the expression $\exp$ and immediately returns a placeholder for the result. The doc parameter is an optional argument for documentation purposes. To resolve the result of the placeholder returned by a future, the placeholder is accessed using the touch construct. The execution of a process performing a touch on the placeholder is blocked until the result of the placeholder is determined.

\subsection{Speculative Computation}

Numerous researchers have proposed various models for speculative computation (Burton [Burton], Miller [Miller], Osbome [Osborne]). We have chosen to use the model proposed by Osborne for the following reasons:

1. The model provides a high degree of expressiveness. It allows the association of priorities with speculative tasks and the grouping of associated speculative lasks logether.

2. The model provides mechanisms that allow the control of rescurces allocated to speculative computation. 
3. The model provides the ability to abort useless computations and the reclamation of resources is implicit.

4. Computations which have been aborted may be restarted if they are found to be required.

In Osbome's model, a task sponsors the activities of another task by expressing a desire for the results of the task. When a task is sponsored, it receives attributes from its sponsor. Attributes dictate how much memory a task may consume and how much processing power the task has at its disposal. The effective attributes of a task are determined by some function which combines the attributes supplied by cach of the task's sponsors. Any change in the effective attributes of a task may in turn lead to changes in the effective attributes of the tasks sponsored by that task.

The priority atribute of a task determines the order in which tasks are given access to resources. The effective priority of a task is determined by assigning it the highest priority attribute supplied by the sponsors of that task. The sponsorship of a task may change (and hence, its priority) due to changes in the attributes of existing sponsors, the addition of new sponsors, and the removal of existing sponsors. The propagation of priorities occurs implicitly in response to changes in sponsorship.

To declare a task irrelevant, we simply remove all of the sponsorship from the task. If a task is unsponsored, it will not run. A nice feature of removing the sponsorship from a task to mark it as irrelevant is that the reclamation is reversible. Should we find that a task that we had marked as irrelevant in actually relevant, then the task may be resumed by its sponsor(s). 
The use of priorities is very simple and they provide a flexible means of expressing the onderings of tasks. When a tast is created, it is assigned a priority at which to execute. As the task is sponsored by other tasks, the task's priority is dynamically adjusted by the system to that of the highest prioriy sponsor.

The base Multilisp language has been extended with a number of new primitives for the support of a speculative style of programming. The primitives may be combined by the programmer to construct more complex procedures, as they are required. For the remainder of this section, we introduce the primitives that have been added to Multilisp for the support of speculative computation and briefly describe their function. In this thesis we refer to the Multilisp language augmented with support for speculative computation as Multilisp+SC.

(spec-future exp pri) - creates a speculative task with priority pri to evaluate expression exp and immediately returns a placeholder for the result.

(make-group exp pri) - performs the same function as spec-future but it returns a group object. A group object consists of a group identifier which is used to reference the task and all of its descendent tasks, and a placeholder for the result of the computations.

(group-id grp) - retums the group identifier of the group object $g r p$.

(group-future grp) - returns the placeholder of the specified group object grp. 
(stay-group 8rp-id) - removes the source sponsorship of all the members of the specified group identifier grp-id.

\subsection{G. als}

This thesis has the following goals:

1. Investigate the potential for speculative computation in improving application response time.

2. Show new examples which illustrate the power of speculative computation.

3. Evaluate and extend the current model for speculative computation.

\subsection{Preview}

Chapter 2 summarizes related work. Chapter 3 describes the general user/interface dialog model used to evaluate the potential of an application for using speculative computing. Chapter 4 describes how different allocations of computing resources can be analyzed to determine their impact on minimizing response times. Chapter 5 introduces methods that can analyze how to best allocate computing resources to minimize the application response time to user requests. Chapter 6 describes the implementation and enforcement of resource allocations using speculative computing. Chapter 7 describes an implementation of the Dungeon Explorer application and presents extensive results to assess the impact of speculative computation on response time. Finally Chapter 8 presents our conclusions and our thuughts on future work. 


\section{Chapter 2}

\section{Related Work}

In this Chapter we dicuss the work of other researchers that is related to the work we have performed in this thesis. We discuss other applications that involve the use of speculative computation to reduce response time, other user interface models and Virtual Reality. Dynamic Programming and Optimal Scheduling as discussed as alternate means to solve the problems that we address in this thesis.

\subsection{Applications}

In this section, we discuss two very different applications involving speculative computation. While neither application is directly concerned with the use of speculative computation to reduce response time, they both demonstrate the potential of speculative computation for reducing both compute and response times. 


\subsubsection{Simulated Annealing}

Simulated annealing is a technique for obtaining approximate solutions to combinatorial optimization problems. The goal of using simulated annealing is to find a solution of minimum cost. The basic search involves three steps:

1. moving from the current solution to a new solution

2. evaluating the cost of the new solution and

3. deciding to accept or reject the new solution to neplace the current solution

Simulated annealing is used to solve problems such as VLSI placement and routing. Computation times on the order of hours or days are typical for these types of problem. The major difficulty in the parallelization of this algorithm is that the algorithm is inherently sequential. Parallel solutions are either very application specific or, by breaking the sequential property of the algorithm, introduce the potential for errors that could not occur in a sequential version. Witte, has successfully used speculative computation to mevixe the run time for this algorithm and has achieved speedup in excess of $\log _{2} P$, on $P$ processors [Witte].

When her algorithm evaluates a current solution to the problem, the algorithm uses speculative computation to evaluate the algorithm for both the case that the current solution is accepted as the new solution and the case that the current solution is rejected.

By speculating on the results of each of the tasks, the search tree can be expanded to arbitrary depth given a sufficient number of processors. The number of processors 
required grows exponentially with the depth of the tree. Witte has addressed this problem by showing that in some instances, we can determine that either the success rate is sizably larger than the failure rate or that the converse is true. This information allows us to distribute our resources for computing successes and failures in an unbalanced fashion. Thus, by allocating mone of our resources to the more probable type of outcome, we effectively increase the depth of our search tree.

Witle has achieved very encouraging speedup figures over conventional sequential approaches. Her algorithm has achieved speedups in excess of $P / 2$ when unbalanced lookahead trees are used and either fallures or successes are highly predictable. Witte has done a fair amount of analysis on what types of speedups can be expected for a given number of processors. While her analysis is not really relevant to our work, it is interesting in its coverage of the "number of processors to speedup" trade off. Her work is concemed with the speedup that can be achieved for specific numbers of processors.

Witte's work is important because it illustrates that by assigning probabilities to the various solution outcomes, we can make more effective use of speculative computation tochniques than we can if all requests are equally probable. While Witue has limited her research to the situation where only two choices are available, we extend the notion of assigning probabilitics to situations where many choices are possible.

\subsubsection{Optimistic Make}

Bubenik and Zwaenepoel [Bubenik] have used the techniques of speculative computing to create a program that they refer to as Optimistic Make. Optimistic Make is a speculative version of the UNIX Make facility [Kernighan]. The purpose of an Optimistic version of 
make is to attempt to compile recently changed files into their executable forms hefore the user actually requests that the files be compiled. If precompilation can be accomplished, then the delay from the time that the user requests the compilation of a file until they can actually execute the file is minimized, thereby improving the user nesponse time.

The primary means by which they achieve optimistic make is through the use of encapsulations. Encapsulations are computations whose outputs are concealed until such time as they are demanded. They use 3 basic encapsulation operations to provide support for optimistic make. Create Encapsulation() creates an encapsulation. It is possible to have an encapsulation read the output of one or more other encapsulations by supplying the identifiers of the other encapsulations as arguments when the encapsulation is created. With MandateEncapsulation(), if the inputs to the cncapsulation are unchanged the outputs of the encapsulation are revealed; otherwise the encapsulation is aborted. AbortEncapsulation() aborts an encapsulation and discards its output. If the outputs of an encapsulation are not mandated within a specified time-out interval, then the encapsulation can be aborted to release system resources. If the user should happen to issue a make request after the encapsulation has been aborted, the computations are then repeated.

Bubenik and Zwaenepoel implemented optimistic make on a network of SUN workstations and found that the average response time speedup over pessimistic make was 8.28 times. Their work was confined to the use of speculative styles of computation, where the computations themselves were quite large-grained. We extend the notion of using speculative computation to decrease response times for situations where the task grain size is not necessarily as large and the precomputations that should be performed to minimize the user response time are not as easy to predict. 


\subsection{User Interface Models}

In this section we discuss User Interface models that are very simular to the model we use in Chapter 3.

\subsubsection{Interactors}

An interesting work that parallels our approach of using interface modeling is that of Faconti and Patemo, in which they specify the basic components from which interactive graphics programs can be modeled [Faconti]. Each component is described as a set of interaction units or interactors. They identify the components of a basic interaction as being input components, output components and control components. An input component is defined as an abstract intemal representation of a possibly complex user input. The output component is the representation of a picture that is in relation with the user input. The control component is responsible for the initialization of the interactor as well as controlling the communication with other interactors in the system. The authors illustrate how to use these components to construct the detailed specification of the interactors within the user interface. This approach has proven to be especially useful in the specification of user interfaces which rely on multi-thread input and on multiple feedback.

The work of Faconti and Paterno has been primarily concerned with using interactors to describe all of the hardware devices and processes that exist with a given user interface system, while omitting the representation of the user component in the system. We have concentrated on how to model the user and the user interface using interactor like objects, without regard to how the functions of the user interface are implemented or the devices 
with which they communicate. Ours is a higher level abstraction of what a user interface represents and how it can be modeled.

\subsubsection{User Interface Modelling using State Machines}

It has been shown that state transition diagrams can be used to model the activities supported by a user interface [Jacob85]. User requests may be represented as stakes in the diagram and the input and output associated with the requests is used to dictate the transition amongst states. The use of state transition diagrams to model a user/interface dialog is useful in that:

1. For each possible state that the dialog may be in, the state transition diagram makes explicit how all possible inputs may be interpreted.

2. State transition diagrams show how interpretations of input to a given state can change depending on the sequence of requests that have resulted in the current state being reached.

3. State transition diagrams emphasize the effect that the user's requests have on the state of the dialog.

A key advantage of employing state transition diagrams is that the diagrams explicit depiction of the transition from one suate to another state allows for the casy identification of the input expected at each state and the resulting output produced. By making the state transition diagram machine readable the application has explicit knowledge of the requests that the user may make. The use of the knowledge conveyed by a machinc readable diagram has been explored by Feyok [Feyok77] to help handle user queries within an online help facility. In Feyok's work the knowledge contained in the diagram allows the 
application to provide the user with the sequences of requests that the user should make to arrive at a specific state.

The use of a state transition diagram to predict the course that the user will follow through a dialog is promising. The use of speculative computation to eagerly compute the output of a request is aided by the fact that the output (and hence the input to another state) from a state is rigidly defined. The model that we use to describe a user interface dialog (discussed in Chapter 3) is based on transition diagrams.

\subsubsection{Properties of a Model for Parallel Computation}

The description and analysis of the computations performed within a parallel program can be simplified by modelling the computations and the execution order of the computations within the program as a graph. Karp and Miller present such a model [Karp66]. In the framework of the model, nodes represent the computation steps within a parallel program and arcs between computation steps represent dependencies between computation steps. The model presented by Karp in Miller is primarily concerned with the sequencing of computation steps and is mathematically rigorous in its analysis of the program graph. In the analysis of the graph model much emphasis is placed on the queuing of data between computation steps. The proof of program determinacy and termination is the central objective of the model. Perhaps one of the most interesting aspects of the model is that it demonstrates how by analyzing the graph model, we can determine the number of times that each computation step within the program will execute.

In relation to the work that we pursue in this thesis, the model presented by Karp and Miller does have numerous deficiencies. The most prominent of these deficiencies is that 
no provision is made for resource limitations (i.e., number of available system processors) or data dependant decisions. While the work of Karp and Miller has been inspirational in its approach to modelling the activities of a parallel program (especially in the area of computation step dependencies), its concentration on data queuing and lack of support for limited resources has limited its direct application to the analysis of a user/interface dialog when the number of processors and data dependant decisions are present.

\subsection{Virtual Reality}

An interesting area of work that could benefit from our rescarch is Virtual Reality. Numerous new applications are being developed in this fast growing field. Many of these new applications employ the use of parallel computers to help achieve adequate response times. The Pixel Planes project at the University of North Carolina consists of over 250,000 processors and is used to render images in real-time [Fuchs]. The Pixel Plancs project is used to render computer graphics at such a speed that the user who views the images feels that they are "immersed" in a new reality. The net effect is that the user feels as though they are part of a movie (not just simply watching one). Other applications such as the GROPE Molecular Simulator use parallel computing coupled with external mechanical devices to help scientists and research make new advances in the field of molecular chemistry by being able to "interact" with the compounds that they create. [Batter]. The processing requirements that Virtual Reality applications require and the emphasis they place on response time makes them very good candidates to benefit from the results of the work we present in the remainder of this thesis. 


\subsection{Dynamic Programming}

In Chapter 3 we present an algorithm and two heuristics that we use to analyze user interface dialogs. Our analysis is done to determine how processing resources should best be allocalod to minimize the expected overall response time. Another means of determining the optimal resource allocation for a particular userfinterface dialog is possible with the aid of dynamic programming [Bellman57].

Dynamic programming is a mathematical means of solving multi-stage decision problems. With dynamic programming we define the solution of a problem in terms of the solution of a recurrence relation. The recurrence relation is solved repeatedly until a solution to decision problem is found. In effect the decison problem is solved backwards from its finish to its start. This approach has a number of advantages over deriving an algorithm from scratch (as we have done in Chapter 3). The primary advantage of using the dynamic programming technique is that an optimal solution can be found without exponential search. In Chapter 5. Section 5.2 we present a possible solution to the optimal scheduling problem that we address in this thesis. In the remainder of this section for the benefit of readers not familiar with dynamic programming we present a brief dynamic programming example*:

Example: The $n^{\text {th }}$ Shonest Path

Sometimes it is of interest to determine not only the shortest path between two locations but also the second shortest, the third shortest and so on. The length of

\footnotetext{
- Breed on example from [Bellman62] pp. 230-231
} 
a path is equal to the sum of the lengths of the ares that connect the nodes on that path. The comparison of the lengths of the various paths can be used to determine how important it is to select the shortest path.

To aid in the derivation of the dynamic programming solution of the problem, let us introduce the seyuence of values:

(1) $v_{i}=$ the time required to go from $i$ to $\mathbf{N}$ using the second shortest path, $i=1$. $2, \ldots, N-1$

In order to obtain a relation for $v i$ it suffices to observe that if a point $j$ is on the second shorrest path from $i$ to $N$ then the portion of the path from $i$ to $j$ is either 1 ) the second shortest path from $i$ to $j$ or 2) the shortest path from $i$ to $j$ and the portion of the path fom $j$ to $N$ is either 1) the shortest or 2) the second shortest. respectively. Clearly, $i$ to $j$ and $j$ to $N$ cannot both be the shortest path; if one of these (say $i$ to $j$ ) was the third shortest path then the original $i$ to $N$ path could not be the second shortest path from $i$ to $N$ (choose the $i$ to $N$ path incorporating the second shortest path from $i$ to $j$ instead).

Let us define two functions min1 and min2:

min $1 w_{i}=$ the absolute minimum of $w_{i}, i=1,2, \ldots, N$

$\min 2 w_{i}=$ the second smallest value of $w_{i}$.

If we restrict our attention to problems with positive are lengths, we have, the equations 
(2) $v_{i}=\min \left\{\min 1\left(t_{i j}+v_{j}\right), \min 2\left(t_{i j}+f j\right)\right\}, i=1,2, \ldots, N-1$ with $v_{N}=\infty$ and $j \neq i \quad j \neq i$

$f i=\min _{j \neq i}\left[t_{i j}+f\right] i=1,2, \ldots, N-1, f N=0$

whene:

$t i j=$ the time required to traverse the path from $i$ to $j$

Similar, but more complex equations can be derived for the nth shortest path.

\subsection{Optimal Scheduling}

The end objective of the analysis of the user/interface dialog graphs is to determine how to best schedule the execution of the nodes so that the expected overall response time is minimized. The idea uf scheduling activities so that the execution time is minimized is not unique to our work. Numerous researchers have studied the intricacies of the sequencing and scheduling of tasks so that the execution time for a collection of tasks is minimized [Baker74, Bruno76].

The intricacies of scheduling tasks is complex and requires the examination of both the predecessors and successors of each task. The complexity of optimally scheduling tasks is further increased by the introduction of the notion of constrained resources [Baker74]. With constrained resources determining the optimal path or shortest critical path is nontrivial.

Our work involves both the scheduling of tasks when resources are constrained and attempting to keep the critical path as short as possible. Our work also posses a further 
restriction in that we also require that the expected overall response time is kept to a minimum. This is identical to attempting to keep the average critical path length as short as possible. In determining the minimum average critical path length, the lengths of the various paths the user can take are weighted by the probability the user will take the path. 


\section{Chapter 3}

\section{A Model For User Interface Analysis}

Determining if the response time of a user/interface dialog may benefit from the use of speculative computation requires the analysis of how the user spends his/her time with the application during the dialog and the structure of the dialog. We also need to know how time is consumed, since if there are no idle periods or the current response times are already very good, then there is no point in attempting to use speculative computation to improve them. As well, we require the analysis of the structure of the application to determine if it is possible to divide the application into segments capable of being executed in parallel.

A model for the analysis of a user/interface dialog, with the objective of identifying those dialogs that could benefit from the use of speculative computation, must include both time and structure components. The model should also be able to represent a wide range of user 
interface styles and complexities. We present a model for analyzing userfinterface dialogs whose properties satisfy these requirements.

We introduce the characteristics of a user/interface dialog (and its supporting platform) that are good indicators of the potential of the application to benefit from speculative computation. We use these characteristics to evaluate the potential of the two example applications introduced in Chapter 1.

\subsection{Graph Model Of Dungeon Explorer Game}

Figure 3.0 is a graphical representation of the Dungeon Explorer game. We refer to this representation of a User/Interface Dialog as a Dialog Graph. Each of the rooms in the game is represented as a node in the dialog graph. Paths lcading from room to room are represented as directed ares, with the direction of the arc representing the direction of the path. Bi-directional paths (i.e., a path that can be traversed in either direction) are represented using two directed arcs, one in each direction. An example of a hi-diructional path connects Room 7 and 8 in Figure 3.0.

Each node in the dialog graph for the Dungeon Explorer game has an associalted compule time and think time. We also lahel each arc in the dialog graph with a prohability. The probability corresponds to the probability that the user will demand the results of the node to which the arc points, given that the user has selected the node from which the arc emanates. As we will see later in Chapter 4, probabilities play an important role in the accurate analysis of a user/interface dialog. 
By modeling a user/intertace dialog as a graph we can learn about the different properties of the dialog that are of interest when deciding if the dialog is suitable for being programmed using speculative computation techniques.

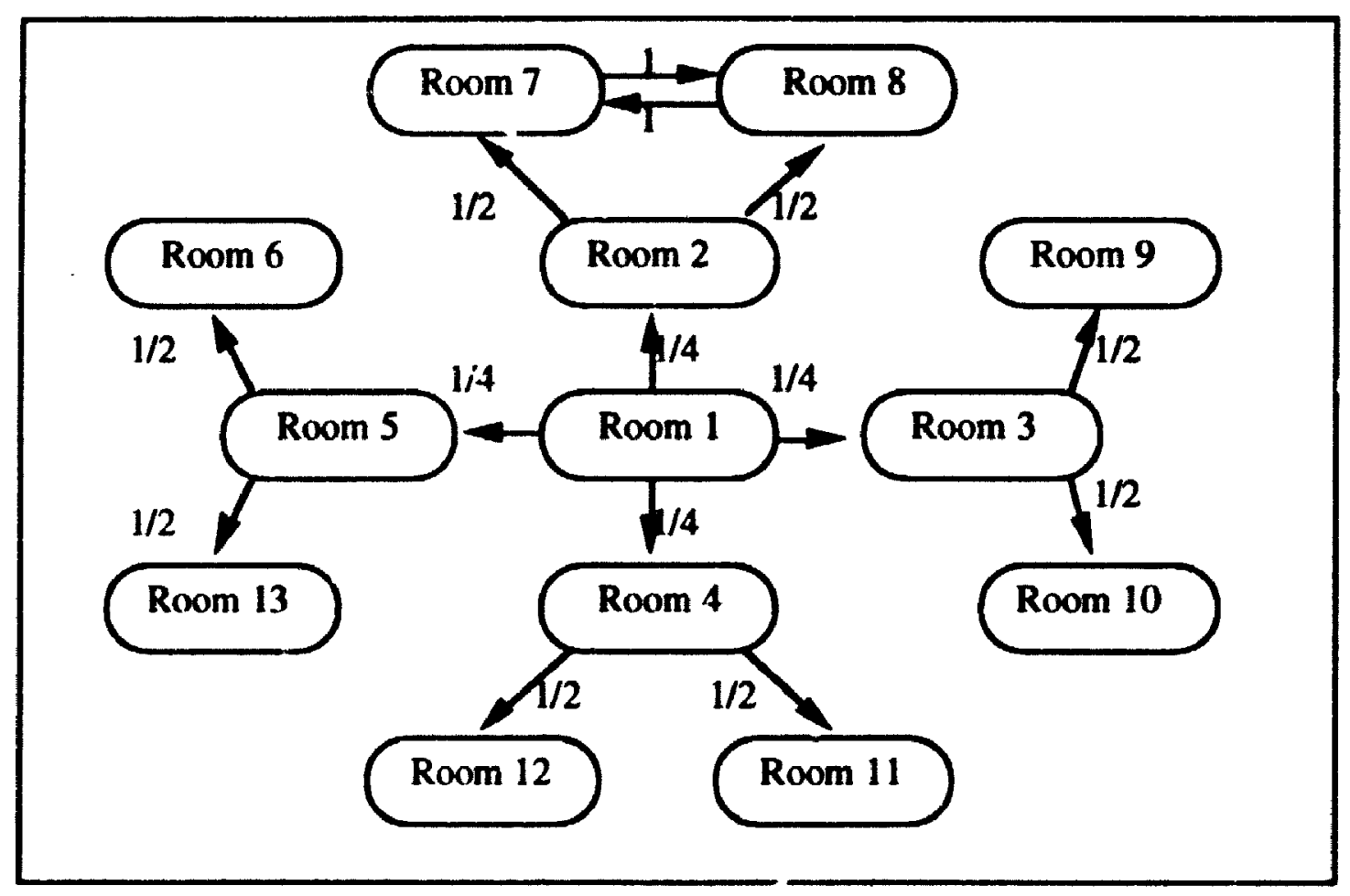

Figure 3.0 - User/Interface Dialog Model for the Dungeon

\section{Exploration Game}

\subsection{The General Model}

Figure 3.1 lists the symbols used to construct our model of a user/interface dialog. In the model we represent requests supported by the interface with hollow nodes. We connect nodes representing two requests that can be made in sequence with a directed arc indicating the ordering of the sequence. A solid node depicts the user's current request. We refer to the node that represents the current request as the focus node, since it represents the fact that the attention of the user/interface dialog is currently "focused" on a request involving 
that node. Since the focus node represents the current activity being negotiated hetween the user and the interface, the actual user component of the interface is implicit within the framework of the model.

\begin{tabular}{|l|c|c|c|}
\hline SYMBOL & O & 0 \\
\hline NAME & Path & Option & Fcrus \\
\hline REPRESENTS & $\begin{array}{c}\text { Direct } \\
\text { Relation }\end{array}$ & Option & $\begin{array}{c}\text { Curnenty } \\
\text { Requested } \\
\text { Option }\end{array}$ \\
\hline
\end{tabular}

Figure 3.1 - Symbols Used in Constructing a User/Interface Dialog Model

During each iteration through the user-interface dialog loop (Figure 3.2), the user makes a request that involves an opticn. The representation of this activity in the model is shown by moving the focus from the location occupied during the previous iteration, to the node representing the newly selected option. Figure 3.3 traces two iterations through the userinterface loop for the dungeon explorer game. Diagram 1, shows that the user is initially in room 1. The first rejyest that the user makes is to move from room 1 to room 2. Diagram 2 depicts this request. We depict the issuance of this user request by moving the focus node from room 1 to room 2. At this point, the application computes the result of the user's request and returns the result to the user by showing him/her room 2 . Once the user has been shown the room, he/she may think for a while about where to move next. Eventually the user decides to request to move from room 2 to room 8 . Again, the application computes the result of the user's request and returns the result to the user, this time showing him/her room 8 . The second iteration ends with the user thinking about where to move next. 


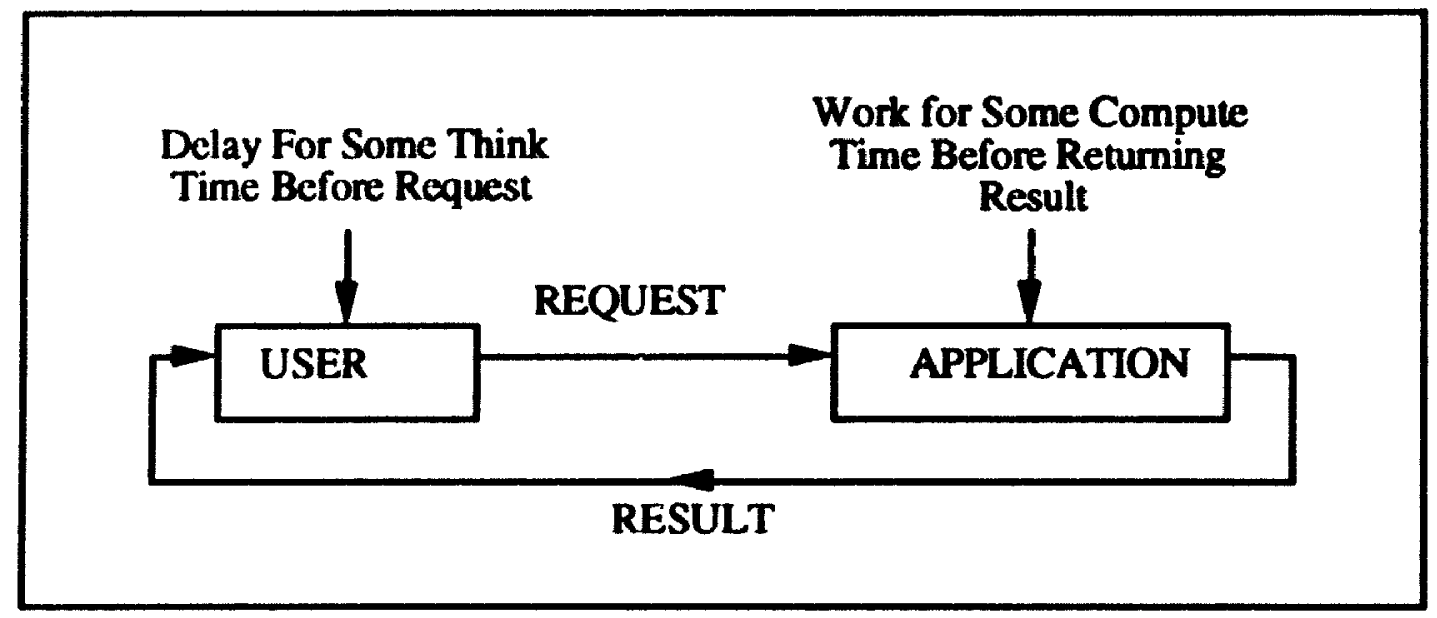

Figure 3.2 - User-Application Dialog Loop

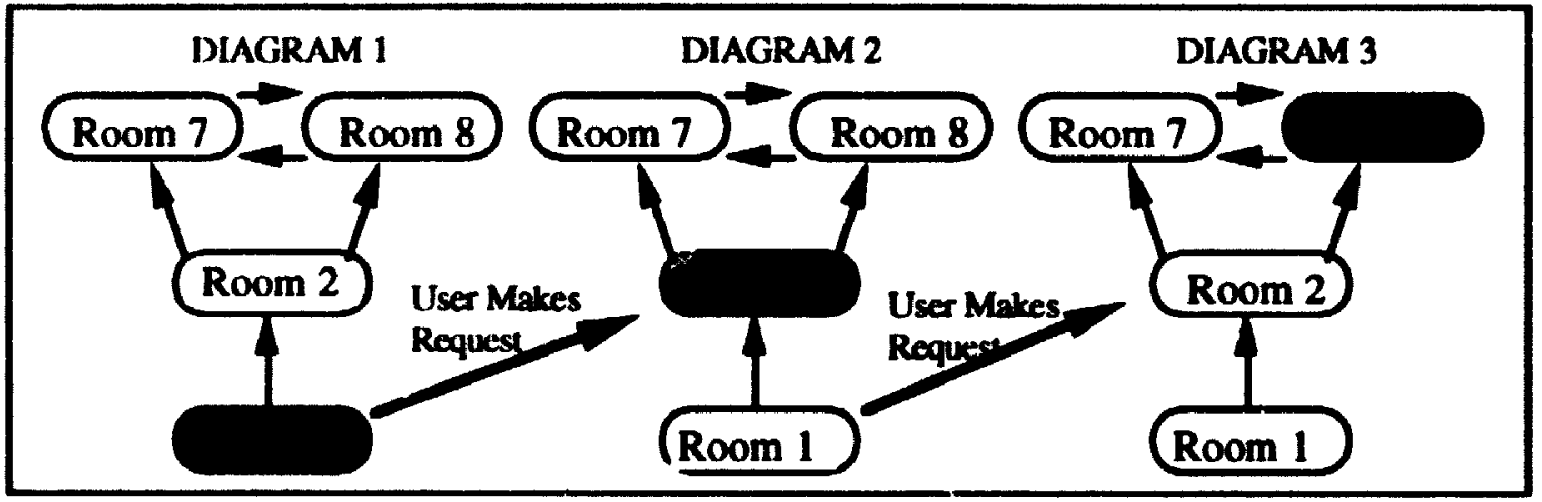

Figure 3.3 - Movement of Focus With New Requests

In the next section we discuss the attributes associated with the nodes and ares in the dialog graph.

\subsection{Dialog Graph Attributes}

Associated with each node in the dialog graph for a user/interface dialog are compute and think time attributes. For many types of requests, it is possible to know exactly how much processing time the request will require. For example, the time it takes to compute and display a room in the Dungeon Explorer game is constant assuming that all rooms have the 
same amount of detail. However, for other types of requests, it may only be possible to specify a time range or distribution for the time required. For example, the time requined to determine if a number is prime or not is not constant for all numbers. The nature of think times makes think times impossible to predict exactly since they are very much a function of the traits of the individual user. To make the model of a dialog more flexible and to allow for more in-depth analysis we allow for the specification of compute and think times as probability distributions.

Associated with each arc in the dialog graph for a user/interface dialog is a probability attribute. U'sually, when a user decides what requests he/she is going to make, the requests are seldom random selections. Requests are, for the most part, a function of the options the user has already selected. Quite often it is easy to predict with a reasonable amount of certainty, what the next request will be. Consider the text editor application: given that the user has just requested the Edit option, we can say that there is a fifty percent chance that he/she will select Copy Text option. We can make that prediction without knowing anything about the user's habits. Perhaps we even know more information because we have observed the user for some time and have found that seventy-five percent of the time that the user requests the Edit option, he/she selects the Copy Text option. By assigning probabilities to the selection of each option, the prediction of options that will be most probably be requested in the near future can be made relative to the position of the user in the dialog graph.

In the next section, we will discuss the general properties of a dialog that can be identified from its dialog graph. 


\subsection{Dialog Graph Properties}

When we examine a dialog graph, there are a number of properties that the graph may exhibit that can be of interest when we consider the suitability of the dialog for being programmed using speculative computation techniques. For our purposes, the three most important properties of the dialog graph are:

1. Does the graph have cycles? If the dialog graph contains cycles, we know that the dialog has the potential to continue forever.

2. Are there multiple paths to a node? If there are multiple paths to a node, this indicates that there are multiple ways that an option can be requested. Depending on which path is taken, the amount of cager computation and the probability of reaching the node may vary.

3. Are the.e nodes that do not have arcs leaving them? These nodes are terminal nodes $r$. sinks and correspond to exit points in the user/interface dialog. If the user demands a request that corresponds to an exit point in the dialog graph, the user is terminating the dialog.

\subsection{Dialog :aph Classifications}

The three propertics introduced in section 3.4 allow us to classify dialogs graphs (and hence the user/interface dialog) as being in one of 3 possible classes: 


\section{Acyclic-Tree}

The dialog graph does not contain cycles and there are no nodes in the graph that are reachable on multiple paths.

\section{Acyclic}

The dialog graph does not contain cycles and there exists at least one node that can be reached on multiple paths.

\section{Cyclic}

The dialog graph contains cycles and thene may exist nodes that can he neached on multiple paths.

With multiple paths, we are presented with the possibility that a node may he visited under different sets of circumstances. If the attributes are always the same regardless of the path. we say that the attributes (think time, compute time) of a node are independent of the path that the node is reached on. If the attributes of a node are dependent on the path, then depending upon which path is taken, the attributes may assume different values. Figure 3.4 illustrates the dependence/independence of paths. In diagram A we illustrate the dependant case; if node $D$ is reached by path ABD, then the Think Time associated with node $D$ is 10. If path ACD is taken then the Think Time associated with node $D$ is 8 . In diagram B we illustrate the independent case; regardless of the path taken to reach node $D$. the Think Time is 10 . 


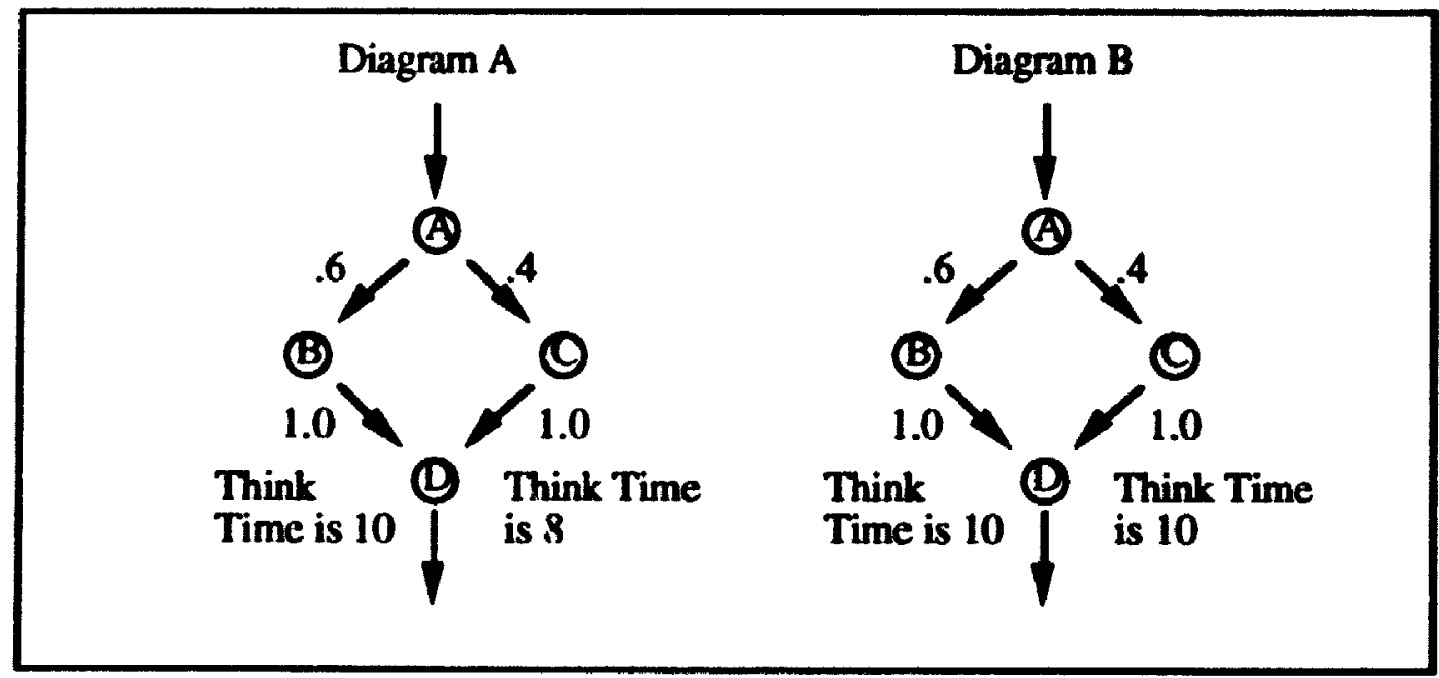

Figure 3.4 - Dependent Versus Independent Paths

Closely related to the dependence/independence of multiple paths is the issue of the dependence/independence of multiple visits to a node when cycles are present. If the visits to a node are independent, the attributes of the node do not change from one visit to the next. If the visits to a node are dependent then the attributes of the node may change from one visit to the next. Figure 3.5 illustrates the dependence/independence of multiple visits. In diagram A, the Compute Time for Node $\mathrm{C}$, changes depending on the number of times that the node is visited. The Compute Time is 10 for the first visit, 5 for the second and 3 for all other repeat visits. In diagram B, the Compute Time for node $C$ is 10 for all visits.

Orthogonal to the classification of nodes as being dependenvindependent, is whether the attributes of the node are time invariant. For instance, the longer the amount of time between the user making a particular request, $A$, the more likely it may become that he/she will make some other request, $B$. If the atuributes of a dialog are time invariant, their values do not change as a function of time during the course of the dialog. 
Diagram A

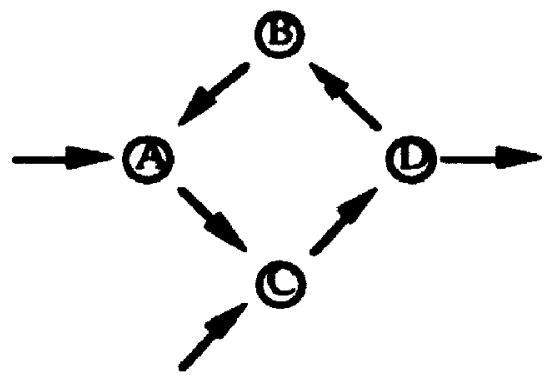

First Visit Compute Time $=10$ Second Visit Compute Time $=5$ All other Visits Compute Time $=3$
Diagram B

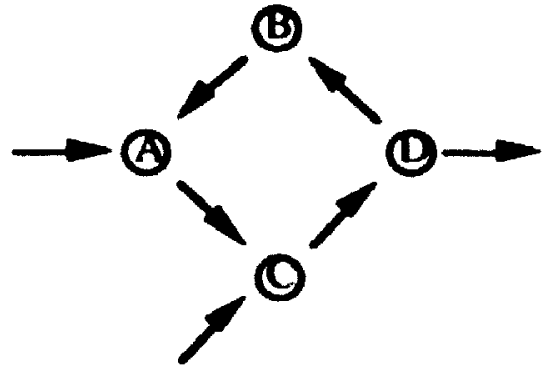

For all Visits Compule Time $=10$

Figure 3.5 - Dependence Versus Independence of Multiple Visits

Dialogs can be further sub-classed as either having an infinite or a finite number of nodes. If the number of unique node instances that comprise a dialog is unbounded, the di? log is infinite. Otherwise, the dialog is finite. Any application which does not have a finite horizon (the user can continually make different requests of the application) is classed as having an infinite number of nodes.

Another aspect of a dialog that can be classified as either finite or infinite, is the length of the sequences of requests that the user can make. Obviously, if the graph has an infinite number of nodes, the number of requests that the user can make during a dialog is possibly infinite. Another possibility for an infinite sequence of requests is if the dialog graph contains cycles. This make it possible that the user could continually make the same set of requests over and over. 
A final sub-classification of dialog graphs is based on how multiple activations of the same node are handled. There are actually two orthogonal ways to classify multiple activations of a node. The first is whether the activations of the node are dependent or independent. The activations can be considered as independent, meaning that the computation of the results of two activations can proceed in parallel. If the computation of the result of activation " $n+1$ " cannot be performed until the result of activation " $n$ " has been computed, then we say that the activations are dependent. The second means of classifying activations is based on whether the computations performed by a node are repeated for each activation of the node. If the results remain constant regardless of the number of activations, the result computed by the node is static. If the results of a node need to be recomputed each time it is activated, the result computed by the node is dynamic.

In this thesis we will restrict our attention to userfinterface dialogs where:

1. Attribute values ane independent of the path taken.

2. Altribute values are independent of the number of times a node is visited.

3. Multiple activations of a node are independent and the results of a node are recomputed for each visit.

4. Request probabilities are independent.

5. Auribute values are time invariant.

6. Finite number of nodes and number of requests.

7. Compute Time and Think Time distributions are discrete.

\subsection{Dialog Graph Reductions}

A large number of the different dialog graph classes can be reduced into the acyclic tree class. Not only is the acyclic tree a desirable graph in a structural sense, but the analysis of 
this type of graph to determine response times (Chapter 4) and the parallel computation of requests (Chapter 5) are both easily solvable. In the following two sections, we present a method that will convert a graph containing multiple paths into an equivalent graph without multiple paths and a method that will convert a cyclic graph into a "reasonably" equivalent acyclic graph.

\subsubsection{Splitting Multiple Nodes}

Multiple paths to nodes can be restructured as single paths to multiple copies of the node. This technique is referred to as node splitting. Figure 3.6 shows an example of how norke splitting can be applied to remove multiple paths to a node from a dialog graph.

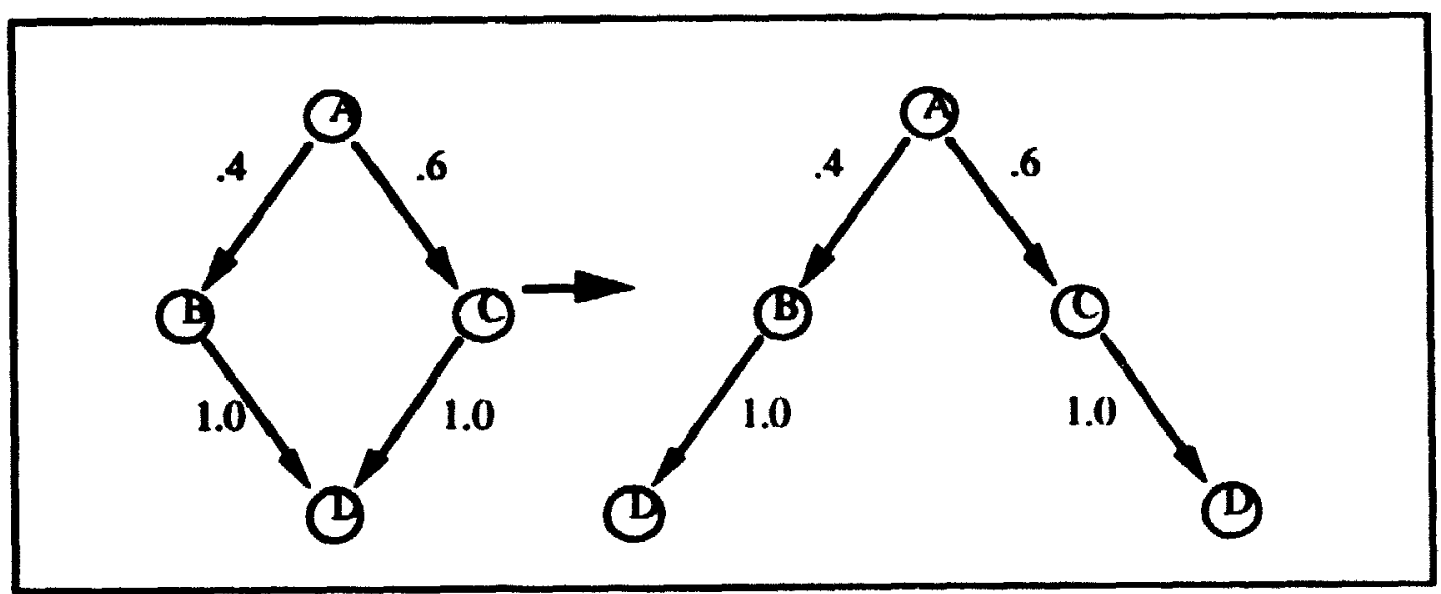

Figure 3.6 - Splitting Nodes in a Graph to Remove Nodes Reachable on Multuple Paths

Node D was reachable in the original graph on paths ABD and ACD. We "split" node D into two copies of itself. We then reconstruct the path using the multiple copics of node D (one for each path) to avoid having a single instance of $D$ that can be reached on two paths. The resulting graph still expresses the same precedence relationships as the original except that the graph is now an acyclic tree instead of cyclic. However, by splitting nodes, we 
have introduced some dependency relations between nodes that did not previously exist. These dependency relations exist between multiple copies of the same node. Any computation performed at one copy of the node is performed al all copies of the node. We represent dependency relations amongst multiple copies of the same node as dashed lines. This technique can be used to restructure graphs where multiple activations of a node ant dependent and the results of a node are not recomputed for each visit. Figure 3.7 shows how the dependencies introduced in the node splitting of the graph in Figure 3.6 are representyd. As we will see in Chapter 5, the dependencies we have introduced though node splituing can be easily handled and do not overly complicate the analysis of the dialog graph.

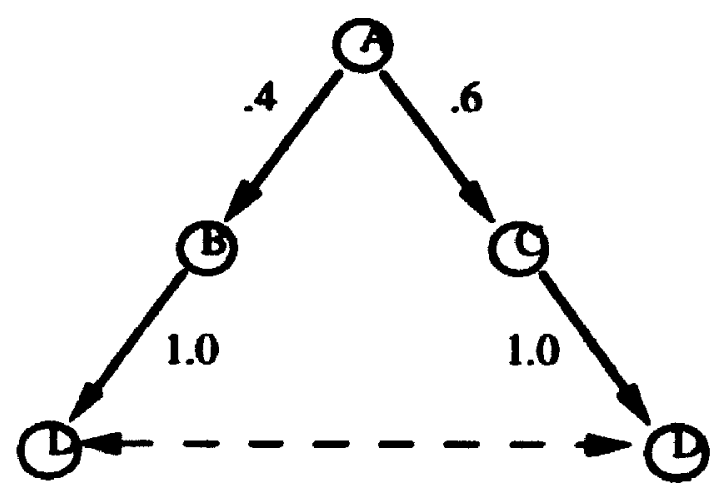

Figure 3.7 - Representing Internode Dependencies that are Introduced by Node Spliting

\subsubsection{Unrolling Cycles}

There are two types of cycles that can exist in a dialog graph: trap and degenerative. A trap cycle is a cycle that once entered by the user cannot be exited. These type of cycles are characterized by the fact that there exists a collection of nodes in the dialog graph containing 
the cycle such that there does not exist an are with non-zero probability that connects a node in the collection to a node outside of the collection. Degenerative cycles are cycles such that the probability that the user continues to loop though the cycle hecomes less and less the more times the user loops through the cycle. These cycles ane characterized by the fact that at least one of the ares in the cycle has a probability less than 1.0.

The process of unrolling a cycle involves the continued expansion of the cycle to cither form a path of infinite length or a path of some fixed length that closely represents the cycle. The length of the fixed length path can either be specified in terms of a constant value, some function of the length of the cycle, or in terms of the probability that the user will continue to loop through the cycle (a tolerance). A constant value can have the undesirable affect of truncating a lengthy cycle before the entire cycle has heen traversed once. However, it does prevent us from continuing to unroll a cycle to an infinite length, and it can prevent us from continuing to follow a cycle that might not be very probable. Unrolling based on the length of the cycle is desirable since we are always assured of unrolling every cycle to some fixed length (in terms of the original length of the cycle). However, we can still he involved in unrolling extremely long cycles, well after the point where the user will probably cease to loop through the cycle. By unrolling based on some specified probability that the user will continue to follow the cycle, we avoid unrolling cycles that may be highly improbable. The potential exists, however, for not unrolling a cycle at all because the probability of the user looping through the cycle once is less than the tolerance. As a rule, we use both a function of the length and a minimum prohability tolerance to control the unrolling of cycles. 


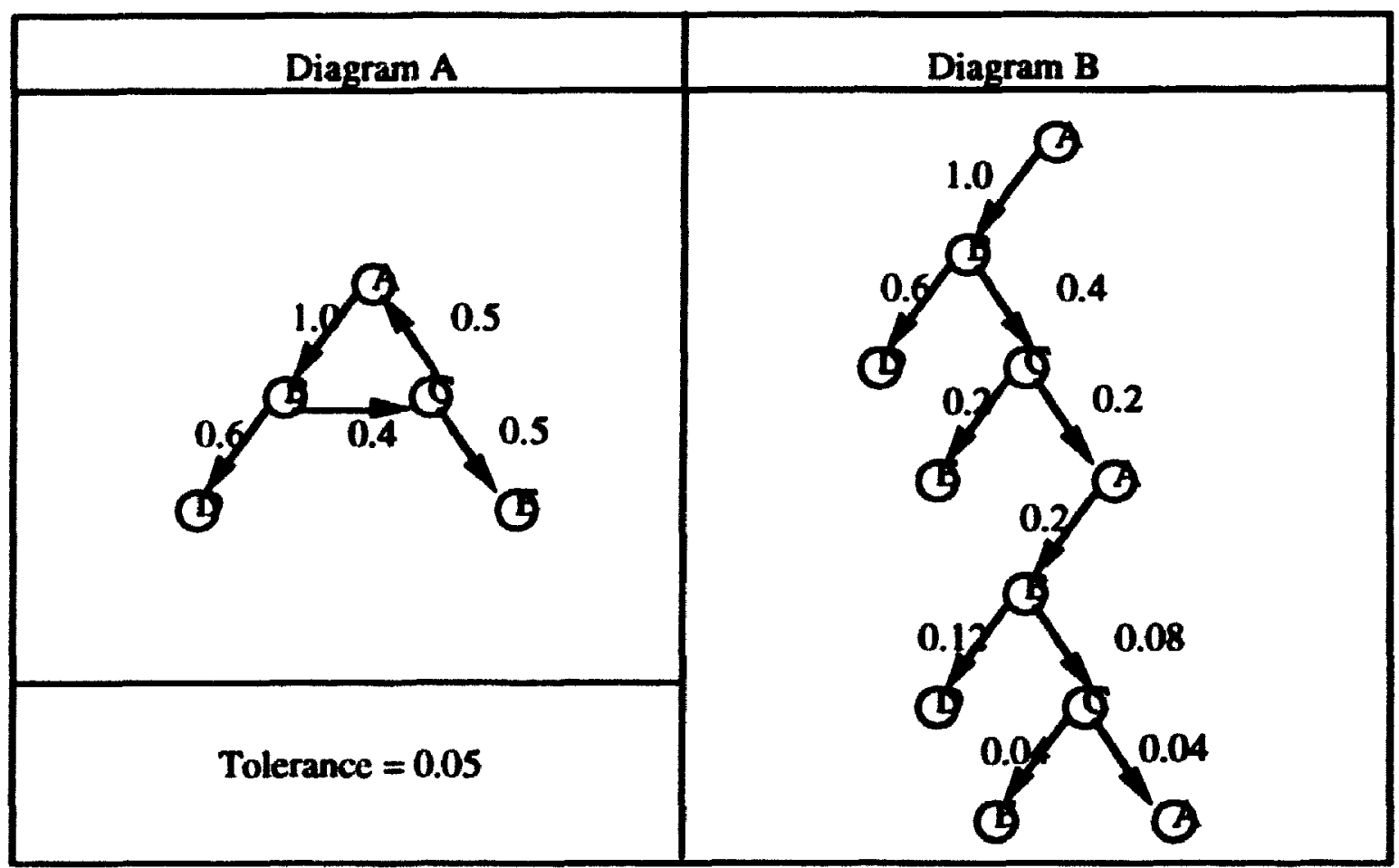

Figure 3.8 - Unrolling A Degenerative Cycle to Form an Acyclic Tree

Figure 3.8 illustrates the unrolling of a graph containing a degenerative cycle to form an acyclic tree dialog graph. We have chosen to unroll the degenerative cycle until the probability that the user continues to loop though the cycle is less than 0.05 . Diagram $A$ shows the original graph and diagram B shows the unrolled version. We terminate the unrolling of the cycle after we encounter node $A$ for the third time. At this point the probability of the user continuing (0.04) has dropped below the tolerance amount.

The act of unrolling cycles to create acyclic approximations of their behavior works well for degenerative cycles. However, trap cycles must be truncated based on their length. This is undesirable since the probability that the user continues to loop through the cycle is still 1.0. An alternative to unrolling trap cycles is to compute their steady state values using Markov models [Molloy]. We can use the steady state probabilities for the cycle in our 
analysis of the dialog instead of the unrolling approximation of the cycle. We will not the pursuing the analysis of trap cycles in this thesis.

\subsection{Constructing a Model}

In this section we describe the procedure that is used to construct the dialog graph for user/interface dialog. We apply the procedure to the Text Editor and the Dungcon Exploner game (described in Chapter 1) to construct their dialog graphs.

\subsubsection{Basic Steps}

There are 5 steps in constructing a model to represent a userfinterface dialog:

\section{Step 1 - Identify Options to Become Nodes in Dialog Graph}

Identify all of the options that the user may access using the user interface for the application.

\section{Step 2 - Identify Options to be Joined With Arcs}

For each of the options identified in step 1, identify those options that may he requested in sequence with it. The identified options will be connceted to the option in the dialog graph.

\section{Step 3 - Assign Probabilities to Arcs}

For each of the arcs introduced in step 2, assign probabilities that represent their chances of being selected in sequence. The probability estimation method employed is left up to the discretion of the model builder. 


\section{Step 4 - Assign Compute Times*}

Estimate the compute time for each of the options. The compute time may be either colsstant or a probability distribution. The assignment of compute times can be non-trivial as a result of parallel computation within the node. This can make the prediction of the compute time difficult

\section{Step 5 - Assign Think Times*}

Estimate the think time for each of the options. The compute time may be either constant or a probability distribution. The assignment of think times are also nontrivial since they are difficult to prefict and vary from user to user.

\subsubsection{Examples of Model Construction}

The follswing two sections describe the construction of the model for the User/Interface Dialog model for the Simple Text Editor and Dungeon Exploration game introduced in Chaptei 1.

\subsubsection{Model for the Simple Text Editor}

We have chosen only to use the first 3 steps in constructing the model for the text editor example. To perform steps 4 and 5 would require the estim tion of the compute and think timcs for the application and they are uni really essential to illustrate the construction of the model.

\footnotetext{
- Stcps 4 and 5 are not required but they can belp to increase the reliability of the analysis results.
} 


\section{Step 1 - Identify Options}

From Figure 1.4, we have identified the following options:

\begin{tabular}{|l|l|l|l|}
\hline Option & Option & Option & Option \\
\hline Enter Text & Edit Text & Delete Text & Copy Text \\
Format Text & Underline & Bold & Italics \\
File & Save & Open & Quit \\
\hline
\end{tabular}

\section{Step 2 - Identify Direct Relations}

For the options listed in Step 1, we have identified the following direct relationships:

\begin{tabular}{|ll|ll|}
\hline Option & Option & Option $\rightarrow$ & Option \\
\hline Enter Text & Edit Text & Format Text & Bold \\
Enter Text & Format Text & Format Text & Italics \\
Enter Text & File & File & Save \\
Edit Text & Copy Text & File & Open \\
Edit Text & Delete Text & File & Quit \\
Format Text & Underline & & \\
\hline
\end{tabular}

\section{Step 3 - Assign Probabilities}

In the assignment of probabilities to options, we assume that the prohability of any accessible option being selected at any given time is equal. 


\begin{tabular}{|ll|c|}
\hline \multicolumn{2}{|c|}{ Relations } & Probability \\
\hline Enter Text & Edit Text & $1 / 3$ \\
Enter Text & Format Text & $1 / 3$ \\
Enter Text & File & $1 / 3$ \\
Edit Text & Copy Text & $1 / 2$ \\
Edit Text & Delete Text & $1 / 2$ \\
Format Text & Underline & $1 / 3$ \\
Format Text & Bold & $1 / 3$ \\
Format Text & Italics & $1 / 3$ \\
File & Save & $1 / 3$ \\
File & Open & $1 / 3$ \\
File & Quit & $1 / 3$ \\
\hline
\end{tabular}

We may now construct the following User/Interface Dialog Graph for the Simple Text Editor: 


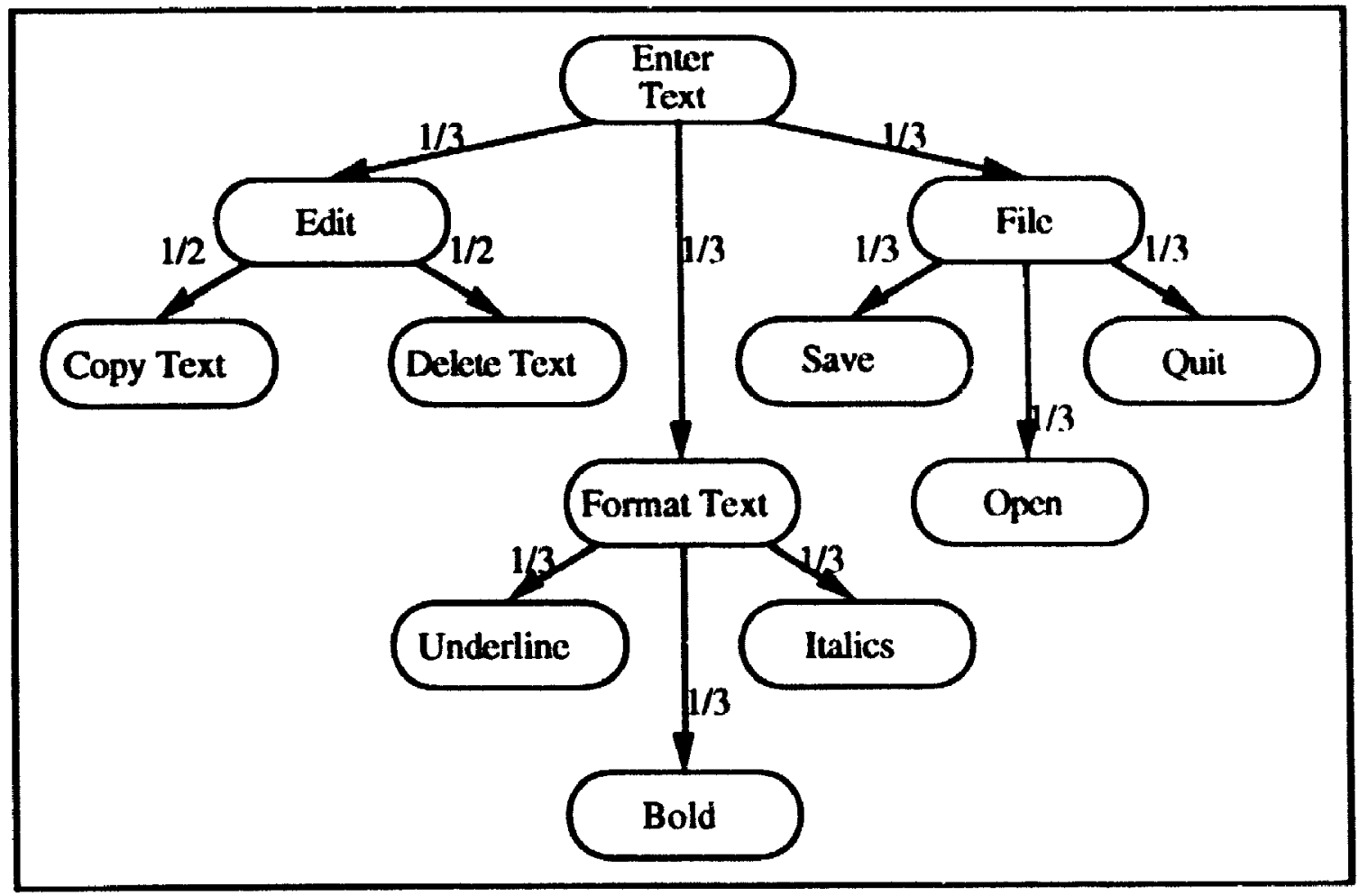

Figure 3.9 - User/Interface Dialog Model of The Simple Text Editor

\subsubsection{Model for the Dungeon Exploration Game}

The option, relations and the probabilities identified for the dungeon exploration game are as follows: 
Step 1 - Identify Options

From Figure 1.4, we may identify the following options:

\begin{tabular}{|l|l|l|l|}
\hline Option & Option & Option & Option \\
\hline Room 1 & Room 2 & Room 3 & Room 4 \\
Room 5 & Room 6 & Room 7 & Room 8 \\
Room 9 & Room 10 & Room 11 & Room 12 \\
Room 13 & & & \\
\hline
\end{tabular}

Step 2 - Identify Direct Relations

For the options listed in Step 2, we may identify the following direct relationships:

\begin{tabular}{|ll|l|ll|}
\hline Option & Option & Option & Option \\
\hline Room 1 & Room 2 & Room 3 & Room 10 \\
Room 1 & Room 3 & Room 4 & Room 11 \\
Room 1 & Room 4 & Room 4 & Room 12 \\
Room 1 & Room 5 & Room 5 & Room 6 \\
Room 2 & Room 7 & Room 5 & Room 13 \\
Room 2 & Room 8 & Room 7 & Room 8 \\
Room 3 & Room 9 & Room 8 & Room 7 \\
\hline
\end{tabular}

Note the depiction of the cyclic relationship between Room 7 and Room 8 as two direct relations, onc for each direction. 


\section{Step 3 - Assign Probabilities}

In the assignment of probabilities to options, we assume that the probability of any accessible option being selected at any given time is equal.

\begin{tabular}{|c|c|c|c|c|c|}
\hline \multicolumn{2}{|c|}{ Relations } & \multirow{2}{*}{$\frac{\text { Prob }}{1 / 4}$} & \multicolumn{2}{|c|}{ Relations } & \multirow{2}{*}{$\frac{\text { Prob }}{1 / 2}$} \\
\hline Room 1 & Room 2 & & Room 3 & Room 10 & \\
\hline Room 1 & Room 3 & $1 / 4$ & Room 4 & Room 11 & $1 / 2$ \\
\hline Room 1 & Room 4 & $1 / 4$ & Room 4 & Room 12 & $1 / 2$ \\
\hline Room 1 & Room 5 & $1 / 4$ & Room 5 & Room 6 & $1 / 2$ \\
\hline Room 2 & Room 7 & $1 / 2$ & Room 5 & Room 13 & $1 / 2$ \\
\hline Room 2 & Poom 8 & $1 / 2$ & Room 7 & Room 8 & 1 \\
\hline Room 3 & Room 9 & $1 / 2$ & Room 8 & Room 7 & 1 \\
\hline
\end{tabular}

For the Dungeon Exploration Game, the User/Interface Dialog Graph, shown in Figure 3.0 (introduced in section 3.1), can be constructed.

\subsection{Characteristics of Speculative Interfaces}

In this section we describe the characteristics a user/irterface dialog must have if it is to benefit from being programmed using speculative computation luchniques.

\subsubsection{Characteristics}

We have determined that speculative computation can be applied to applications to decrease the response times to user requests. Through experiment we have found a minimum set of 
requirements that an application/platform must meet before being programmed using speculative computation.

\section{Inherent Application Characteristics}

\section{Compute Requests in Parallel}

It must be possible for the results of requests to be computed in parallel.

\section{Predict Likely Requests}

It must be possible to predict which options are likely to be requested at any given time. Without being able to do this, it is impossible to use resources to efficiently reduce response time. In addition to being able to predict requests, it is also important that the number of requests possible at any time is small, or as a minimum, that several highly probable requests are present.

\section{Performance Gain Offsets Start-up Costs}

The amount of processing time that can be spent eagerly computing the result of a request must be sufficient to offset the start-up costs and ine costs of possibly having to halt the computing if the request is found to be irrelevant. If the start-up time required to create a speculative task to compute the result of a request is greatcr than the potential performance gain achievable from eagerly computing the result, the response time will be worse. 


\section{Platform Characteristics}

\section{Idle Resources}

The platform that the application is going to be run on must have idle resources. If the machine is already saturated with mandatory computation, then speculative computation will not be useful.

If one or more of these characteristics are absent from a user/interface dialog, this a good indicator that the application will not benefit from being programmed using speculative techniques. Obviously, if a large percentage of the total options or the more probahle options have these characteristics, the application may still be a good candidate.

\subsubsection{Evaluation of Examples}

The following two sections evaluate the suitability of the Simple Text Editor and Dungeon Exploration game for being programmed using speculative computation techniques.

\subsubsection{Evaluation of the Simple Text Editor}

With respect to the requirements of using speculative computation to improve the response time of an application, the Simple Text Editor example evaluates as follows:

\section{Compute Requests in Parallel}

It is difficult to compute the results of some requests in parallel since they involve input from other requests. For example, it is not possible w compuke the result of saving a file until the file name is known. 


\section{Predict Likely Requests}

It is possible to predict which requests are most likely to be made at any given point in time. For example, the most probable request that will be made is to enter text.

\section{Performance Gain Offsets Start-up Costs}

The types of requests that the user makes while using the text editor are very simple requests (i.e., enter text, underline, delete). The eager computation of these requests would not result in performance gains of the magnitude that would offset the costs of the overhead associated with eagerly computing their results.

As can be scen from the evaluation of the Simple Text Editor example, the characteristics of the types of requests that ane made by the user are not satisfactory for the purposes of using speculative computation to improve response time. The two main aspects of the text editor that prevent it from being a good candidate are that the requests that the user makes of the application do not require a great deal of processing and the requests often involve input parameters from the user that affect how they are performed.

\subsubsection{Evaluation of the Dungeon Exploration Game}

With respect to the requirements of using speculative computation to improve the response time of an application, the Dungeon Exploration Game example evaluates as follows: 


\section{Compute Requests in Parallel}

It is possible to compute the majority of the requests in parallel, since, for the most part, they are independent from each other. For example. the computation of what the user will see by moving into one of three rooms can proceed in parallel since the views they present are all independent.

\section{Predict Likely Requests}

It is possible to predict which requests are most likely to be made at any given point in time. For example, if the user is currently in room 4, we can say, if the way he/she chooses a room to enter is unbiased, then he/she will enter room 12. with $50 \%$ certainty.

\section{Performance Gain Offsets Start-up Costs}

The types of request that the user makes while using the Dungeon Exploration Game result in substantial amounts of processing to render the images of the next room. The time required to render an image should more that offset the cost of eagerly performing the computations involved.

As can bee seen from the evaluation of the Dungeon Exploration game, the application is a good candidate for the use of speculative computation to improve response time. The computations required by the user requests are independent and resource intensive. The requests that the user will make at a given point in the dialog are also predictable, allowing for the more efficient speculation of user requests. 


\subsection{Summary}

In this chapler, we have proposed a model that can be used as a means of representing a user/interface dialog as a graph. The purpose of this model is to allow for the analysis of a user interface to determine if it can benefit from being programmed using speculative computation. The model also provides for the clear representation of those aspects of a user/interface dialog that can be used to determine how resources should be applied to the model to reduce the user request response time. 


\section{Chapter 4}

\section{Calculating Response Time}

In Chapter 3, we showed how to analyze a user/interface dialog to determine if the dialog could benefit from specuiative computation. This process involved modeling the user/interface dialog as a graph. In this chapter, we describe how to measure the potential response time improvement of executing the nodes that comprise the graph in a given order. We also describe how to analyze the groups of nodes that are exceuted in parallel to determine how much time the user spends waiting for the interface to compute the results of his/her request.

\subsection{Introduction}

This section provides information about the motivation for the uchniques developed, as well as some background information. We will use Figure 4.0 extensively in this chapter 
to introduce the various techniques that we have developed to analyze the graph representations of a user/interface dialog for calculating response time. The numbers shown inside the hollow nodes are the option numbers. For example, if we have selected option 0 then we may either select option 1 or option 2 next.

The purpose of the developed techniques is to determine how to allocate resources to best minimize the response times. In our analysis we will assume that we know the Compute time. Think time, and probability for each of the nodes and that the dialog graph has been reduced using the techniques presented in Chapter 3, such that each node has exactly one path leading to it.

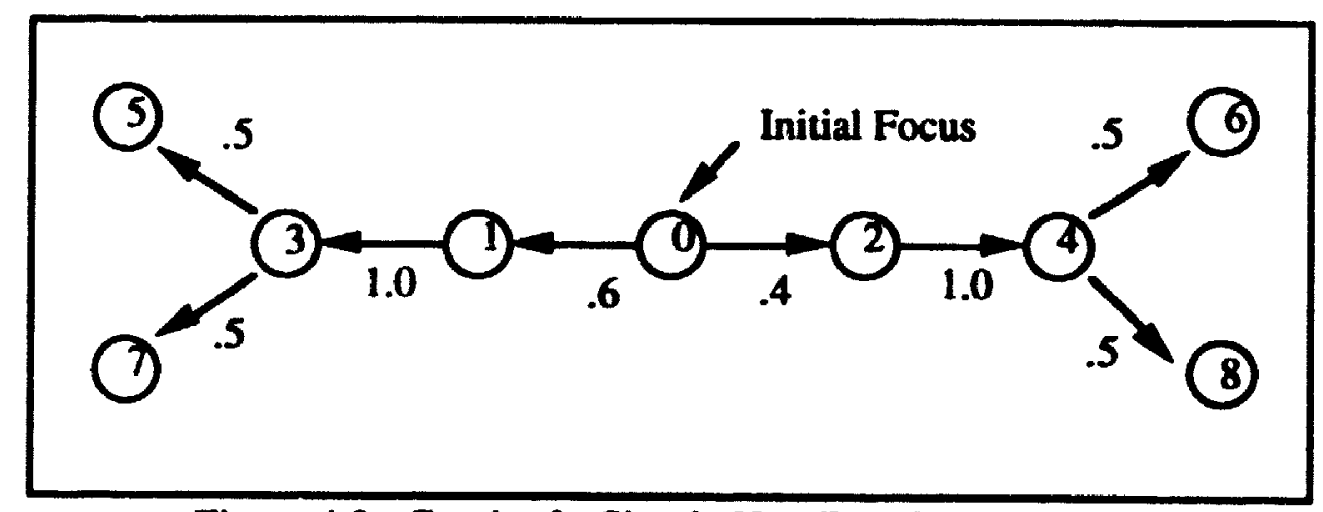

Figure 4.0 - Graph of a Simple User/Interface Dialog

In order to simplify the andysis of a dialog graph, we have chosen to assume that each task requires the same number of processors. This constraint can be relaxed but at the cost of added analysis complexity. However, with revisions, the analysis techniques that we present in this chapter can be extended to situations where the number of privecessors requined per task is not constant. 
When we speak of how many processors are available to an application, we ane neferring to the number of virtual processors available to that application. not the number of physical processors available. A set of physical processors may he represented as a virtual processor. So, for example, if the generation/display of an image requires 4 processors and we say that the system has 8 processors, what we are actually stating is that the system has 2 virtual processors, each of which consists of 4 physical processors.

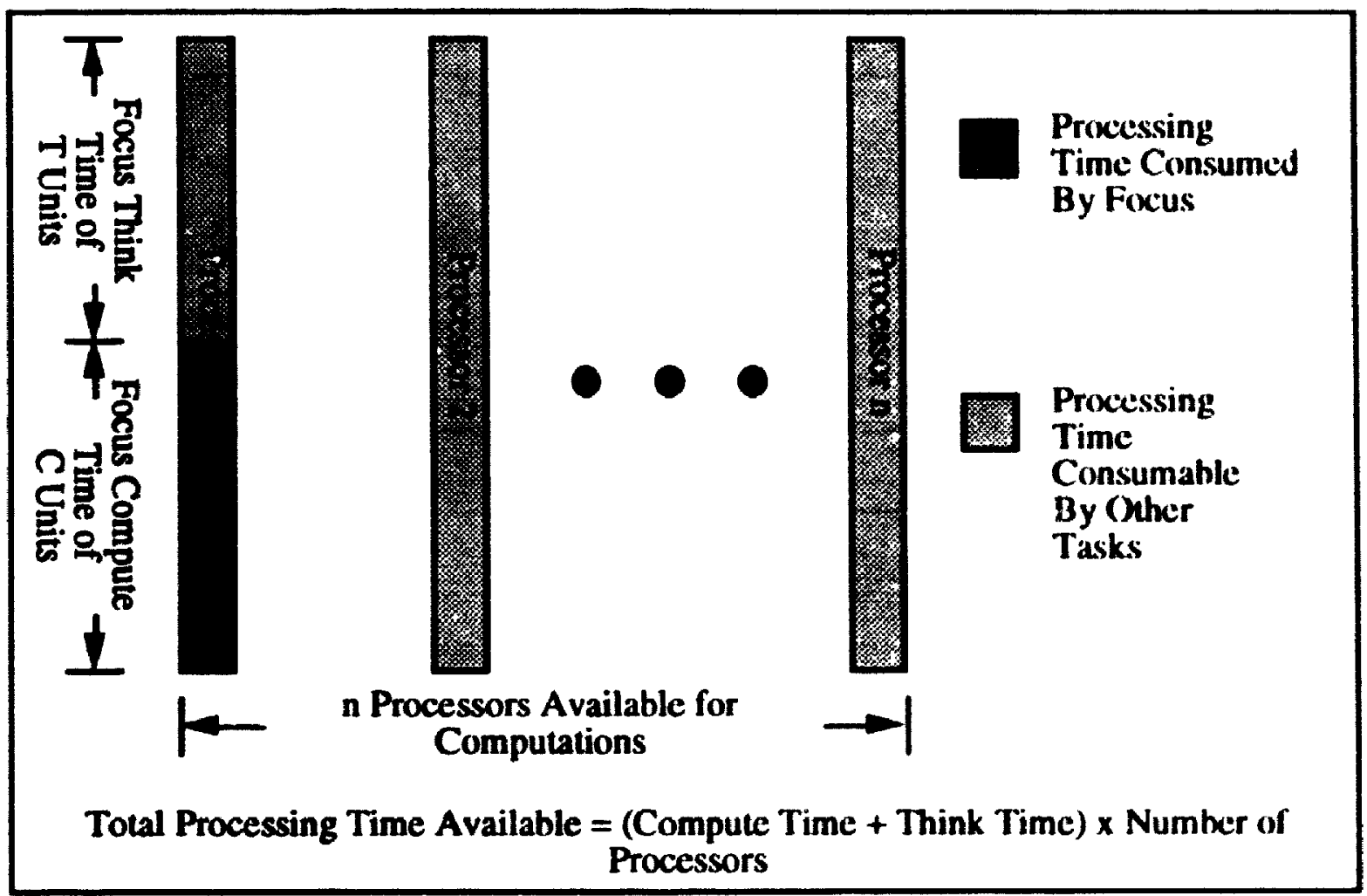

Figure 4.1 - Computation of Total Processing Time Available 


\subsection{Request Groups}

We have already shown how to model a user/interface dialog as a graph, where the requests are nodes and each has an associated comnute time and think thïe. When the application evaluates a request, we may also wish to execute at the same time, some other requests that we are not sure will be required. The Request Group of a node consists of those nodes that we wish to execute in parallel with that node, should the results of the node be requested by the user.

As showr: in Figure 4.1, the consumption of processing time by the node and all of the members of its request group cannot exceed the total amount of processing time generated by the evaluation of the focus node:

\section{Total Processing Time Available $=($ Compute Time + Think Time $) *$ Number of Processors}

We model the allocation of processing time to the members of the request group by the ficus node allocating fixed amounts of time to each of the members of its request group. The Request Group associated with cach node is of the form:

$$
\text { Group }=\{(\text { Node, Processing Time }) \ldots(\text { Node, Processing Time })\}
$$

Node is the name of the node and Processing Time is the amount of processing time that the nude is to receive. We include the notion of a processing time allotment for the completeness of the definition of a request group and for greater control over the 
allocation of resources. As we will discuss in Chapter 5, the specification of processing time allotments is important in the solution of the Bin-Packing problem.

Since we may not be able to compute the result of a node by the end of a single processing time allotment, it is possible that the node may need to the a member of several different request groups. The Eager Compute Time for a noje is equal to the total amount of computation previously done at a node before the user demands the result of the node. We define the Expected Eager Compute Time of a node to be the amount of computation on that node's request that we expect to accomplish before the user demands the result of the node.

We also stipulate that once the result of a node is computed, the node not appear in any other request groups and its result will remain present in the system until demanded by the user or found irrelevant. In Chapter 5, we will show how to creale the groups for cach node. In this chapter, we will only concern ourselves with the analysis of existing groups and their creation.

\subsection{Expected Response Time}

The response time of an interface to a request is the time required to return the result to the user from the time that the user makes the request. With our analysis wehniques we are not trying to minimize the response time for the next request that the user may make. Instead, we are trying to minimize the overall response time for the dialog. If we were only to try to minimize the response time for the next request, we would only need to allocate processing time to those nodes that would be direct descendants of the focus. 
Instcad, when we attempt to minimize the overall resporse time for the dialog, we must consider the impact of the resource allocation with respect to the overall response time and not just the user's current request. For example, if we dedicated all of our resources to the most probable request, we rnight find that this results in the response time for that request being minimized at the expense of some other requests that will have no eager computation performed on them.

\subsection{Calculating the Overall Expected Response Time}

Minimizing the overall response time for a dialog is the same as minimizing the total amount of time that the user has to spend waiting for the results of his/her requests over the course of the dialog. This amount of time is equal to the sum of the delay times for each of the requests that the user makes:

Overall Response Time $=$ Delay $\left(\right.$ Request $\left._{1}\right)+\ldots+$ Delay $\left(\right.$ Request $\left._{n}\right)$

However, the calculation of the overall response time can only be done once the user has completed the dialog.

An estimation of the overall response time of the dialog, the expected overall response time (E[ORT]), is possible if we know:

1. The initial location of the focus. 
2. The relative probability, $P_{r e l}$, of each request being made, given the current position of the focus. The relative probability of a request is the probability that the user will demand the result of the computation associated with the request, given the current locaticn of the user within the dialog.

3. The Compute Time $\left(T_{C_{i}}\right)$ and Eager Compute Time $\left(T_{C_{i}}\right)$ for each request. Since we have restricted our work to include only those dialogs for which the corresponding dialog graph is a directed acyclic trex, the total eager compute time for each request is the total amount of processing time allocated to that node before it becomes the focus.

4. The number of processors $(P)$.

5. The request groups associated with each of the nodes in the user/interface dialog graph.

If we know these 5 pieces of information, then we can calculate the expected delay time $\left(E\left[T_{d}\right]\right)$ that each node will contribute to the overall time the user spends waiting for results. The $E\left[T_{d}\right]$ of Node, $\mathrm{N}_{\mathrm{i}}$, can be calculated as follows:

$$
E\left[T_{d}\right]_{i}=\left(T_{c i}-T_{e_{i}}\right) * P_{\text {reli }}
$$

The expected overall response time for any set of request groups for a graph can then be calculated as the sum of the expected delay times for each of the nodes in the model: 


$$
E[O R T]=E\left[T_{d l 0}+E\left[T_{d}\right]_{1}+\ldots+E\left[T_{d}\right]_{n}\right.
$$

For example, consider Figure 4.0: Assume the focus is located at node 0 , the number of processors is 1 , the compute time for each node is 1.0 , and the think time for each node is 0.0. Since the number of processors is 1 and the think times are zero, it is not possible to eagerly compute any nodes since all the processing resources will be required to compute the focus

$$
\begin{aligned}
& E[O R T]=E\left[T_{d l 0}+E\left[T_{d} l_{1}+E\left[T_{d}\right]_{2}+E\left[T_{d}\right]_{3}+E\left[T_{d}\right]_{4}+E\left[T_{d}\right]_{5}+\right.\right. \\
& E\left[T_{d} l_{6}+E\left[T_{d}\right]_{7}+E\left[T_{d}\right]_{8}\right. \\
& =P_{\text {rel0 } 0} *\left(T_{c_{0}}-T_{c_{0}}\right)+P_{r_{e l}} *\left(T_{c_{1}}-T_{e_{1}}\right)+P_{\text {reb }} *\left(T_{c_{2}}-T_{e_{2}}\right)+ \\
& \text { Prel3 }^{*}\left(T_{\mathrm{C}_{3}}-\mathrm{T}_{\mathrm{e3}}\right)+\mathrm{P}_{\mathrm{rel}} *\left(\mathrm{~T}_{\mathrm{c4}}-\mathrm{T}_{\mathrm{e} 4}\right)+\mathrm{P}_{\mathrm{rel} 5} *\left(\mathrm{~T}_{\mathrm{c5}}-\mathrm{T}_{\mathrm{e} 5}\right)+ \\
& P_{\mathrm{rel}_{6}} *\left(\mathrm{~T}_{\mathrm{C}_{6}}-\mathrm{T}_{\mathrm{e6}}\right)+\mathrm{P}_{\mathrm{reh}} *\left(\mathrm{~T}_{\mathrm{c7}}-\mathrm{T}_{\mathrm{e} 7}\right)+\mathrm{P}_{\mathrm{rel} 8} *\left(\mathrm{~T}_{\mathrm{c8}}-\mathrm{T}_{\mathrm{e8}}\right) \\
& =1.0(1-0)+.6(1-0)+.4(1-0)+.6(1-0)+.4(1-0)+.3(1-0)+.2(1-0)+ \\
& .3(1-0)+.2(1-0) \\
& =4.0
\end{aligned}
$$

When we have more than one processor available, the groups associated with each node will be non-empty. Since we are executing some nodes in parallel, we should see a decrease in the overall expected response time.

Consider the following example which compares the impact of 2 different resource allocations (Figure 4.2 and Figure 4.3) on the E[ORT] for the user/interface dialog degicted in Figure 4.0. In this example we are assuming that the number of processors is 3. the compute time for each node is 1 and the think time is 0 . For each case, we have 
shown the compute time, the request group and the expected eager compute time associated with each node. The format of the request group for each node is a list of 2tuples whose first element is the node ID and the second element is the amoun! of rrocessing time that is allocated to that node. The node request groups shown in Figure 4.2 result in an expected overall response time of 2.0 which is superior to the 2.2 expected overall response time for Figure 4.3.

The importance of this example is that it illustrates how the analysis techniques can be used to determine how good a partitioning of a user interface dialog into parallel segments is, at reducing the overall expected response time for a dialog. We will make extensive use of this procedure for evaluating groups in Chapter 5. Appendix A contains the pseudo code for an algorithm to compute the E[ORT] of a dialog graph for a given set of request groups. 


\section{Case 1:}

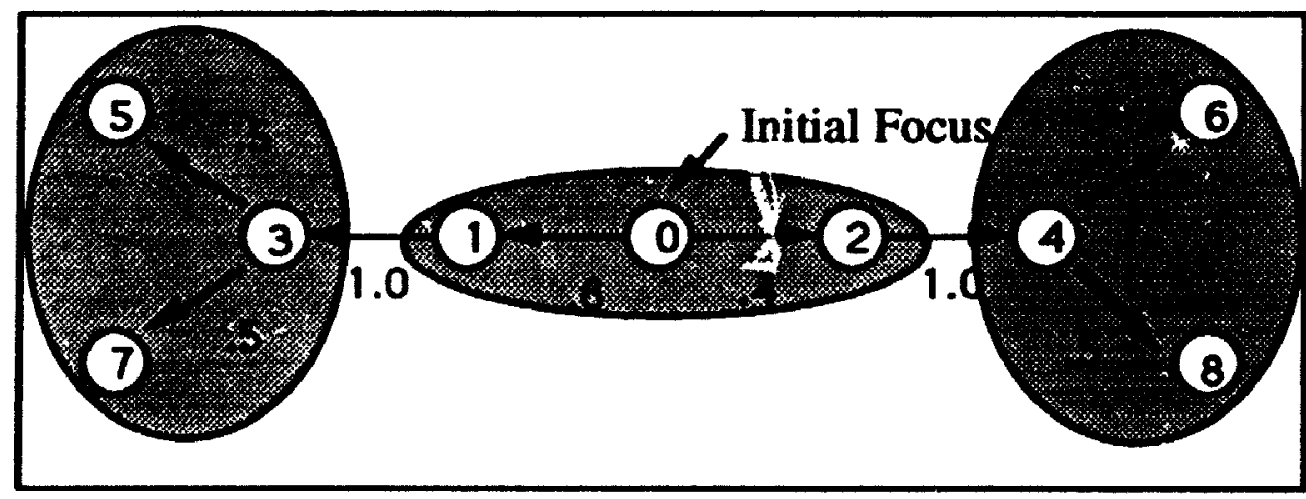

Figure 4.2 - Graph of a Simple User/Interface Dialog

\begin{tabular}{|c|c|c|c|}
\hline Node & $\begin{array}{c}\text { Compute } \\
\text { Time }\end{array}$ & $\begin{array}{c}\text { Request } \\
\text { Group }\end{array}$ & $\begin{array}{c}\text { Eager } \\
\text { Compute } \\
\text { Time }\end{array}$ \\
\hline 0 & 1.0 & $\{(1,1),(2,1)\}$ & 0.0 \\
1 & 1.0 & \{\} & 1.0 \\
2 & 1.0 & \{\} & 1.0 \\
3 & 1.0 & $\{(5,1),(7,1)\}$ & 0.0 \\
4 & 1.0 & $\{(6,1),(8,1)\}$ & 0.0 \\
5 & 1.0 & \{\} & 1.0 \\
6 & 1.0 & \{\} & 1.0 \\
7 & 1.0 & \{\} & 1.0 \\
8 & 1.0 & \{\} & 1.0 \\
\hline
\end{tabular}

$$
\begin{aligned}
E[O R T]= & E\left[T_{d} l_{0}+E\left[T_{d}\right]_{1}+E\left[T_{d}\right]_{2}+E\left[T_{d}\right]_{3}+E\left[T_{d}\right]_{4} r E\left[T_{d}\right]_{5}+\right. \\
& E\left[T_{d}\right]_{6}+E\left[T_{d}\right]_{7}+E\left[T_{d}\right]_{8} \\
= & 1.0 *(1-0)+0.6 *(1-1)+0.4 *(1-1)+0.6 *(1-0)+0.4 *(1-0)+ \\
& 0.3 *(1-1)+0.2 *(1-1)+0.3 *(1-1)+0.2 *(1-1) \\
= & 2.0
\end{aligned}
$$




\section{Case 2:}

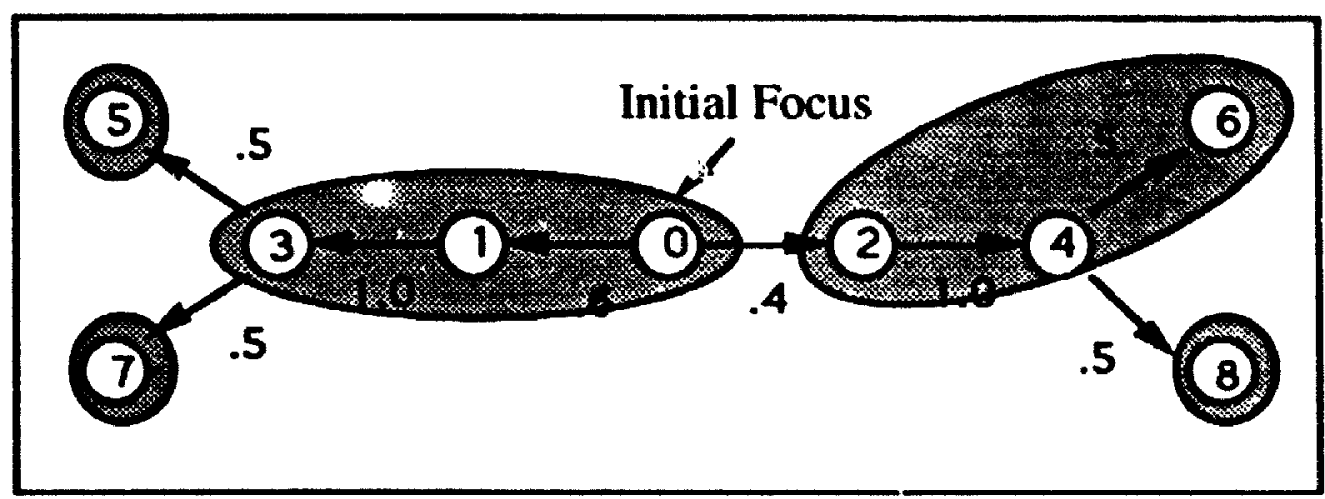

Figure 4.3 - Graph of a Simple User/Interface Dialog

\begin{tabular}{|c|c|c|c|}
\hline Node & $\begin{array}{c}\text { Compute } \\
\text { Time }\end{array}$ & $\begin{array}{c}\text { Request } \\
\text { Group }\end{array}$ & $\begin{array}{c}\text { Eager } \\
\text { Compute } \\
\text { Time }\end{array}$ \\
\hline 0 & 1.0 & $\{(1,1),(3,1)\}$ & 0.0 \\
1 & 1.0 & \{\} & 1.0 \\
2 & 1.0 & $\{(4,1),(6,1)\}$ & 0.0 \\
3 & 1.0 & \{\} & 1.0 \\
4 & 1.0 & \{\} & 1.0 \\
5 & 1.0 & \{\} & 0.0 \\
6 & 1.0 & \{\} & 1.0 \\
7 & 1.0 & \{\} & 0.0 \\
8 & 1.0 & \{\} & 0.0 \\
\hline
\end{tabular}

$$
\begin{aligned}
E[O R T]= & E\left[T_{d} l_{0}+E\left[T_{d}\right]_{1}+E\left[T_{d}\right]_{2}+E\left[T_{d}\right]_{3}+E\left[T_{d} l_{4}+E\left[T_{d}\right]_{5}+\right.\right. \\
& E\left[T_{d} l_{6}+E\left[T_{d} l_{7}+E\left[T_{d}\right]_{8}\right.\right. \\
= & 1.0 *(1-0)+0.6 *(1-1)+0.4 *(1-0)+0.6 *(1-1)+0.4 *(1-1)+ \\
& 0.3 *(1-0)+0.2 *(1-1)+0.3 *(1-0)+0.2 *(1-0) \\
= & 2.2
\end{aligned}
$$


CHAPIIR 4

CALCULATING RESPONSE TIME

PAGE 65

4.5 Summary

In this chapter we have shown how the graph structure for the representation of a uscr/interface dialog, allows us to group user requests for execution in parallel. We have shown how these groups can be analyzed to determine the expected overall response time for a user/interface dialog. The use of the techniques developed will be very important in the next chapter, where they are used to evaluate request groups to determine the optimal group. 


\section{Chapter 5}

\section{Constructing Request Groups}

In our analysis of a user/interface dialog graph to determine the overall expected response time, we assumed that each node had an associated request group consisting of those nodes that were to be executed in parallel with the node should its results be requested hy the user. In this Chapter we present an algorithm which calculates these groups such that the overall expected response time is minimized. We analyze the characteristics of the algorithm to determine some heuristics. As a result of this analysis we presemt iwo heuristics that yield request groups that result in overall expected response times approaching those predicted by the algorithm. We analyze these heuristics to determine formulae for the speedup achievable using the request groups constructed hy these heuristics.

We assume during the introduction of the algorithm and the heuristics that the compute and think times for each node in the graph is known and constant, that cach node is a single 
task, and that no other computation is competing for the processor. After the methods have buen introduced, we consider the impact of inexact compute and think times.

\subsection{The Problem}

We want to construct the request groups for each node such that the overall expected response time for the dialog is minimized. In order to construct node request groups that result in the minimum expected response times, we must consider four factors:

1. The number of processors which are available.

2. The probabilities of each of the requests.

3. The amount of processing time which is available to group members. This is a function of the number of processors, the compute time, and think time of the focus.

4. The structure of the dialog graph.

These factors affect the request group associated with each node and the group/groups to which a node belongs. To simplify the analysis of the problem, we assume that the number of processors which are available to the system is known at run-time. Knowing the compute and think times allows us to determine exactly what order and for how long to execule each request group member so that the most important requests are always excecuting. This allows us to ensure that any available resources are only allocated to those requests with the highest likelihood of being required.

The compule and think times of the focus also impact the degree that we can minimize the overall expecked response time. We may think of the focus node as producing processing 
time for the members of its request group to consume. For cach unit of compute time, the focus produces P-1 units of processing time for its group members (where $P$ is the number of available processors). Since the focus does not require the use of a processor while the user is deciding what request to make next, the focus produces $P$ units of processing time for each unit of think time required by the uscr. The total amount of processing time (Sponsorship Units) which a node can use to sponsor the activities of the me'mbers of its request group is:

\section{Sponsorship Units $=$ Compute Time $*($ Number of Processors -1$)+$ Think Time * Number of Processors}

When we construct a request group for a node we must obscrve that the following thre: constraints hold:

1. The total amount of time allocated to the members of the group dixes not exceed the number of sponsorship units provided by the focus.

2. No group member receives more than the sum of the Compute and Think Times of the focus in sponsorship units. It is not possible for the focus to allocate more units to a member of its request group than it can make availahle to that member on a single processor.

3. No group $m$, nber can execute on more than one processor simultaneously.

If these three constraints are met, we are not guaranteed to have groupings such that the overall expected response time is minimal, but we are guaranteed that the sponsorship unit

\footnotetext{
- We use the term sponsor to refer to the act of supplying processing time to the members of its request group.
} 
allocations specified can be granted. The formulation of the groups such that the overall response time is minimal is the subject of the following sections.

In order to simplify the analysis of dialogs, we will assume that the dialog graphs that we analyze have had the reductions presented in Chapter 3 applied; hence these graphs are acyclic-trees.

\subsection{Generating Optimal Groupings}

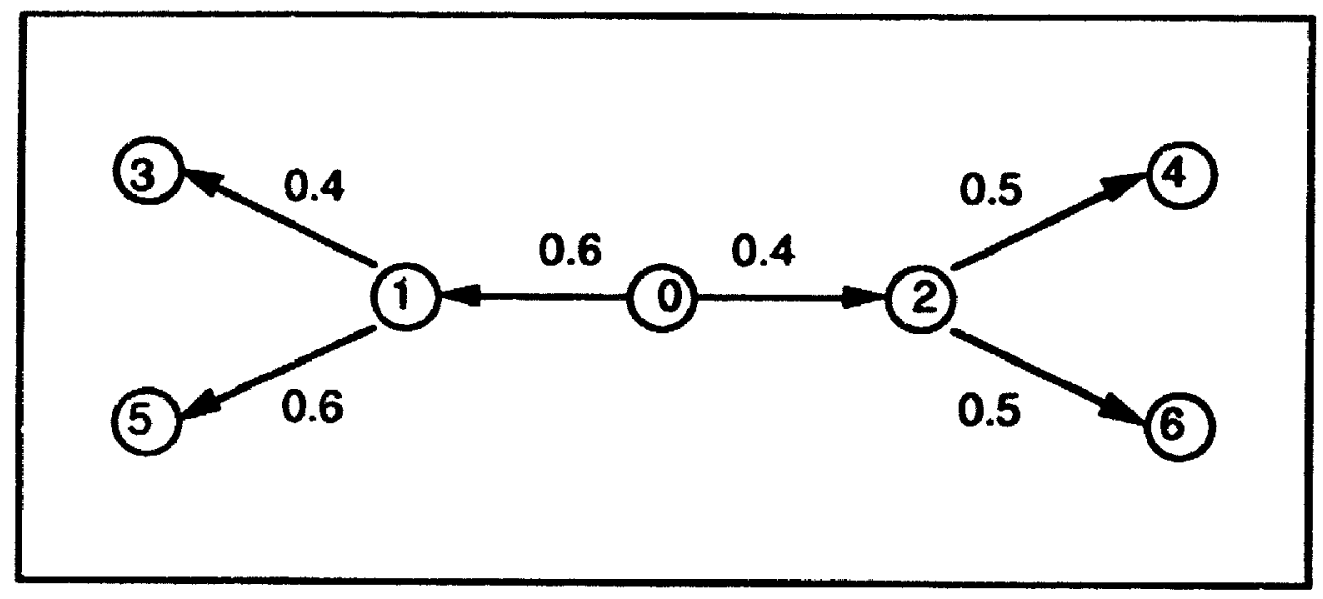

Figure 5.0 -- Simple User/Interface Dialog Graph with Compute Time $=1$ and Think Time $=0$ For All Nodes

The algorithm for gencrating optimal groupings is guaranteed to construct the set of request groups for any user/interface dialog that will result in the minimum overall expected response timc. This is accomplished by constructing all the different request groups for cisch node and then determining which combination of groups result in the lowest experted overall response time. The algorithm to determine the optimal combination of groups, which we refer to as the Request Group Construction (RGC) algorithm, is as follows: 
1. Let Min_E[ORT] represent the minimum E[ORT] calculated for the dialog. Lit

Min_Comb represent the combination which results in the minimum E[ORT].

Initialize Min_E[ORT $]=$ INFINITY, Min_Comb $=$ nil

2. Until all the combinations of request groups have bein evaluatid

2.1 Generate the Next Combination

2.2 Compute the E[ORT] for the Combination (as described in (hapter 4 )

2.3 If the E[ORT] computed for the new combination is less than Min_E[ORT] then Min_E[ORT] $=E[O R T]$ and Min_Comb $=$ Combination

\section{Return Min_Comb}

For Figure 5.0 with 3 processors, the following groups would he generaled and evalualed by the algsrithm:

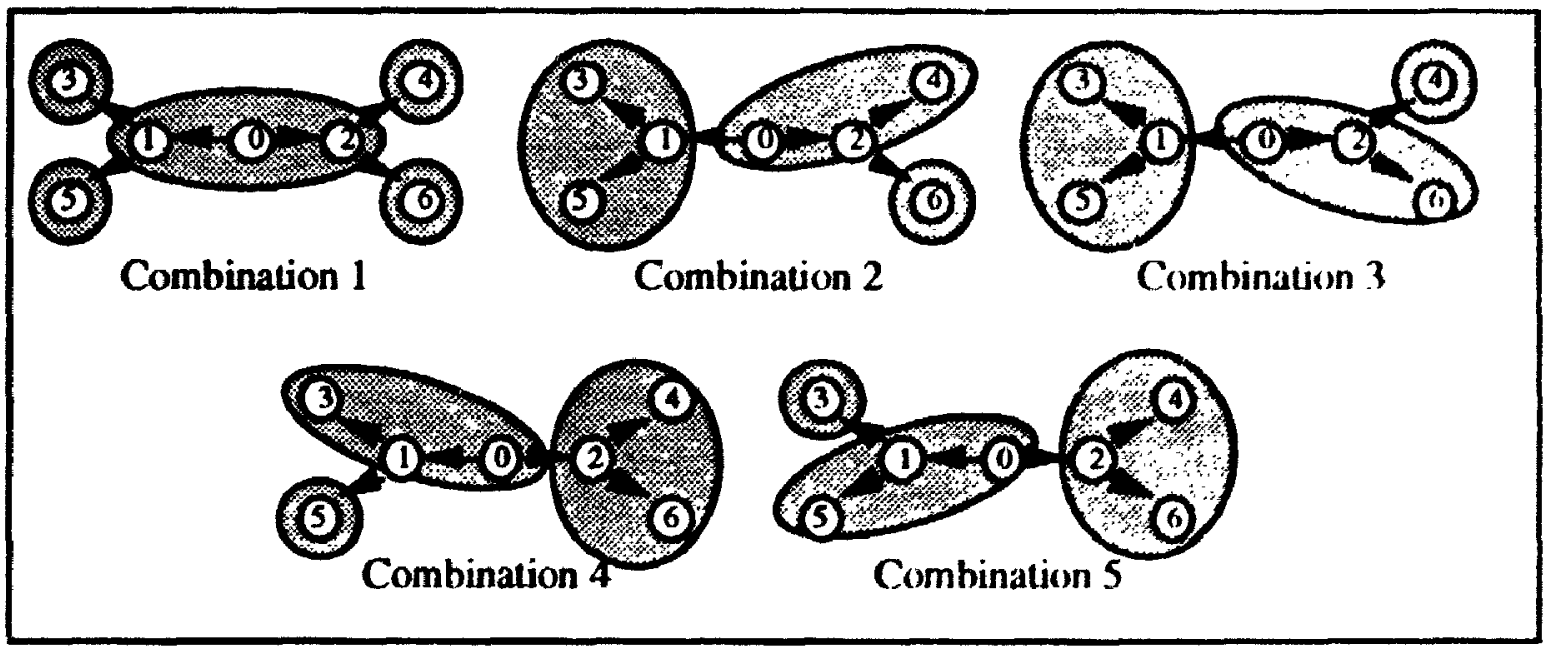

\begin{tabular}{|c|c|c|c|c|c|c|c|c|}
\hline \multirow{7}{*}{$\begin{array}{c}\text { Combin- } \\
\text { ations } \\
1 \\
2 \\
3 \\
4 \\
5\end{array}$} & \multicolumn{8}{|c|}{ Request Groupings } \\
\hline & & 1 & 2 & 3 & 4 & & & $E \mid(O R T)$ \\
\hline & $\{(1,1),(2,1)\}$ & & \{\} & (I) & \{1 & (1) & (1) & \\
\hline & $\{(2,1),(4,1)\}$ & $\{(3,1),(5,1)\}$ & if & (j) & ij & ii & ii & 1.8 \\
\hline & $\{(2,1),(6,1)\}$ & $\{(3,1),(5,1)\}$ & & & \{\} & if & if & 1.8 \\
\hline & $\{(1,1),(3,1)\}$ & & {$[(4,1),(6,1)\}$} & u & if & ii & i) & 1.76 \\
\hline & $\{(1,1),(3,1)\}$ & (i) & {$[(4,1),(6,1)\}$} & if & ii & if & ij & 1.64 \\
\hline
\end{tabular}


The minimum overall expected response time calculated was 1.64 , so the algorithm would return combination number 5 as its result.

As can he observed from the example, for even a relatively simple graph the algorithm needs to evaluate a large number of groups to arrive at an optimal solution. In fact, this is a major deficiency with the algorithm. For most graphs* amount of time taken to construct the final grouping $\&$, ws exponentially as the average number of branches per node ixcreases and combinatorially as the average number of nodes in the graph increases. The construction time also grows combinatorially as the number of processors involved increases. These two factors make the algorithm unfeasible for use in situations where the number of processors and/or the number of nodes in the model is large or the number of processors available can vary dynamically from request to request.

We can casily reduce the size of the state space by making the observation that when we construct request groups, the group should contain only nodes that are reachable from the focus of group. This statement is obvious as we are trying to minimize the expected overall response time and this ohjective cannot be achieved by using resources to compute the result of nodes that are node reachable from the current focus. This state space reduction, while allowing for the analysis of more complex graphs, still does not provide suitable support analysis of graphs when a large number of processors or nodes are considered.

As we discussed in Chapter 2, Dynamic Programming is another technique that could be used to exctract an exact solution to the minimum response time problem. We can

\footnotetext{
" For a graph in which cach mode has cxactly one descendent, the addition of a single node only results in a lifacar increase in lime taken to arrive at a solution.
} 
formulate the mathematical characterization of the decision problem we consider in this thesis as folows:

Let:

$R_{i}(v)=$ the expected execution time at node $i$, where $v$ is a vector of all the compute time remaining for all tasks.

To formulate a recurrence relation for $R_{i}(v)$ we must specify $R_{i}(v)$ in terms of the execution times of all the possible descendar! nodes:

$[1] R_{i}(v)=\underset{j}{\Sigma}\left(P_{j} *\left[F_{i}+R_{j}\left(v^{\prime}\right)\right]\right)$

[assuming decision $\mathrm{d}$ has heen made ahout what tasks to schedule at node: where:

$\mathbf{P}_{\mathbf{j}}=$ the probability that node $\mathbf{j}$ is chosen

$F_{i}=$ the execution time required hy node i to complete its execution

$v^{\prime}=$ vector $\mathrm{v}$ of all the compute time remaining for all tusks resulting from decision $d$

Note: This formula is valid only for instances involving deterministic execution times.

The minimum expected overall execution time for node i may be defined as:

[2] minimum $\left[R_{\mathbf{j}}(\mathbf{v})\right]$ of all possible $d$. 
Using the above definition for $\mathrm{R}_{\mathrm{i}}(\mathrm{v})$, a solution to [1] and [2] represents an optimal allocation given that we want to minimize the expected overall response time.

As we present in Section 5.2.2 even with the favorable results that may be gained from the use of dynamic programing we have chosen to develop specialized heuristics for computing request groups. We did this because we felt that the dynamic programming approach was to complex to implement. Since we only use the output from the RGC algorithm as a muans to measure the accuracy of our heuristics, achieving the highest computational speed for the algorithm is not critical to our work.

\subsubsection{Analysis}

In this section we discuss the difficulties of determining a general formula to compute the expected overall response time that would result from the application of the RGC algorithm. We present formulac for computing the expected overall response time for graphs that have specific structural/probability characteristics. We also present two instances of dialog graph structures that exhibit the rather unintuitive nature of calculating optimal groupings.

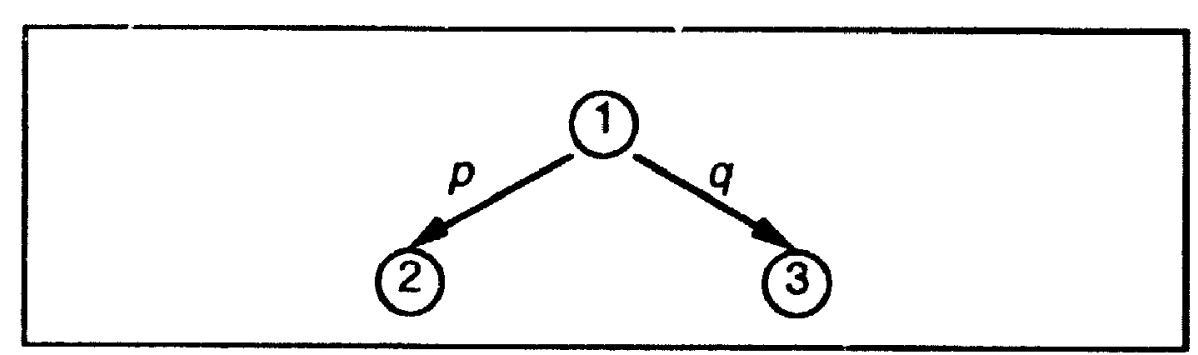

Figure 5.1 - Simple Dialog Graph 
We can construct equations for the overall expected response time for a dialog graph. These equations tend to be very complicated if we do not place nestrictions on the values that the compute and think times can assume. If we use the dialog graph shown in Figure 5.1 and assume deterministic compute and think times (where $C_{i}$ is the compule time firr node $i$ and $T_{i}$ is the think time for node : we can derive the following cquations.

For 1 Processor:

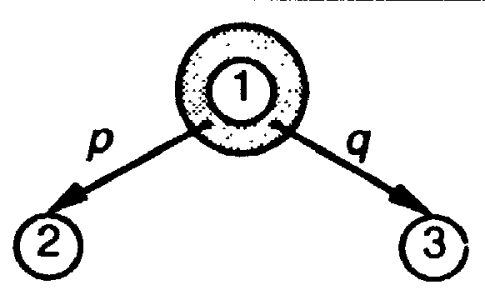

$$
\begin{array}{r}
E[O R T]=C_{1}+T_{1}+\min \left(p^{*} \max \left(C_{2}-T_{1}, 0\right)+q^{*} \max \left(C_{3}-\max \left(T_{1}-C_{2},(1),(0),\right.\right.\right. \\
q^{*} \max \left(C_{3}-T_{1}, 0\right)+p^{*} \max \left(C_{2} \cdot \max \left(T_{1}-C_{3},(0),(0)\right)\right.
\end{array}
$$

For 2 Processors:

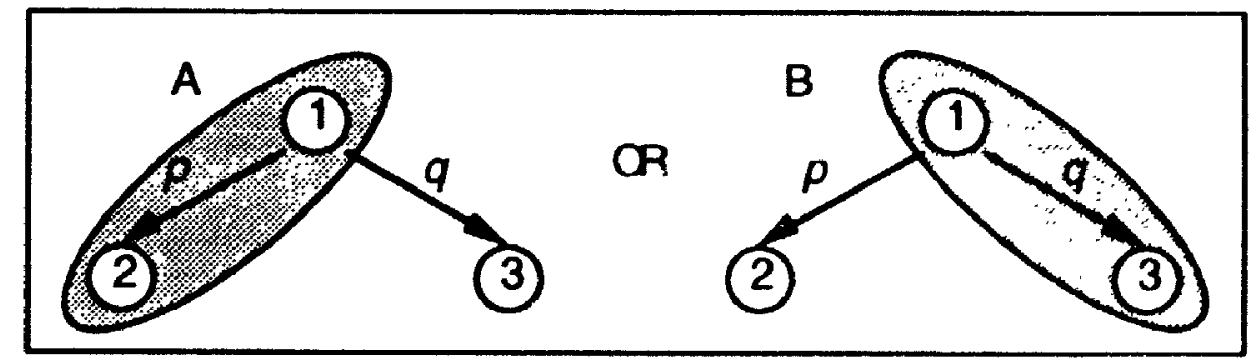

$$
\begin{aligned}
& E[O R T]=C_{1}+T 1+ \\
& \qquad \begin{array}{l}
\min \left(p^{*} \max \left(C_{2}-T_{1}-C_{1}, 0\right)+q^{*} \max \left(C_{3}-T_{1}-\max \left(C_{j}-C_{2},(0),(1), \mid A\right]\right.\right. \\
q^{*} \max \left(C_{3}-T_{1}-C_{1}, 0\right)+p^{*} \max \left(C_{2}-T_{1}-\max \left(C_{1}-C_{3},(1),(0)\right)|B|\right.
\end{array}
\end{aligned}
$$

For 3 Processors: 


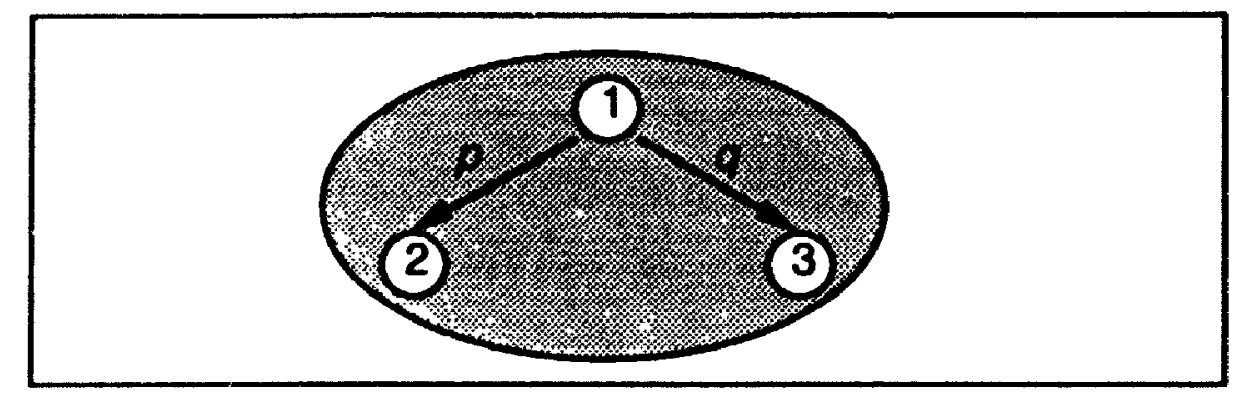

I: $[O R T)=\max \left(C_{1}+T_{1}, C_{2}, C_{3}\right)$

Allowing the compute and think time associated with a node to be represented by a probability distribution increases the complexity if the E[ORT] equation. If we introduce prohabilistic compute and think times, the equations for the above situations would become:

For 1 Processor:

$$
\begin{array}{r}
E\left[O R^{\prime} T\right]=\iiint \int_{C_{1} C_{2} C_{3} C_{4}}\left\{C_{1}+T_{1}+\min \left(p^{*} \max \left(C_{2}-T_{1}, 0\right)+q^{*} \max \left(C_{3}-\max \left(T_{1}-C_{2}, 0\right), 0\right),\right.\right. \\
\left.\left.q^{*} \max \left(C_{3}-T_{1}, 0\right)+p^{*} \max \left(C_{2}-\max \left(T_{1}-C_{3}, 0\right), 0\right)\right)\right\} \\
f_{C_{1}}\left(C_{1}\right)^{*} f_{C_{2}}\left(C_{2}\right)^{*} f_{C_{3}}\left(C_{3}\right)^{*} f_{T_{1}}\left(T_{1}\right)^{*} d C_{1} d C_{2} d C_{3} d T_{1}
\end{array}
$$

For 2 Processurs:

$$
\begin{gathered}
E[O R T]=\iiint \int_{C_{1} C_{3} C_{4}}\left\{C_{1}+\min \left(p^{*} \max \left(C_{2}-T_{1}-C_{1}, 0\right)+q^{*} \max \left(C_{3}-\left|C_{2}-C_{1}\right|-T_{1}, 0\right),\right.\right. \\
\left.q^{*} \max \left(C_{3}-T_{1}-C_{1}, 0\right)+p^{*} \max \left(C_{2}-\left|C_{3}-C_{1}\right|-T_{1}, 0\right)\right\}^{*} \\
f_{C_{1}}\left(C_{1}\right)^{*}{ }_{C_{2}}\left(C_{2}\right)^{*} f_{C_{3}}\left(C_{3}\right)^{*} f_{T_{1}}\left(T_{1}\right)^{*} d C_{1} d C_{2} d C_{3} d T_{1}
\end{gathered}
$$

For 3 Processors:

$$
\mathrm{E}[\mathrm{ORT}]=\iiint_{C_{1}\left(C_{2} C_{3} C_{4}\right.}\left\{\max \left(C_{1}+T_{1}, C_{2}, C_{3}\right)\right\}^{*} \mathrm{f}_{C_{1}}\left(C_{1}\right)^{*} f_{C_{2}}\left(C_{2}\right)^{*} f_{C_{3}}\left(C_{3}\right)^{*} f_{T_{1}}\left(T_{1}\right)^{*}
$$




$$
\mathrm{dC}_{1} \mathrm{dC}_{2} \mathrm{dC}_{3} \mathrm{dT}_{1}
$$

As we can see, the complexity of these equations, even for such a simple dialog graph, is prohibitive. The complexity is a direct result of the need to record the state of all individual tasks. It should be obvious that solving such equations for even moderately complex graphs is almost hopeless. We can alleviate some of the complexity hy assuming that cach node requires the same constant time to execute and that the think time is $\%$ ro. We refer to these types of graphs as CCZT's (Constant Compute Zero Think). If these assumptions are made we only need to record the state of each group. For constant compute time and zero think times the equations for the graph in Figure 5.1 reduce to:

For 1 Processor:

$$
\mathrm{E}[\mathrm{ORT}]=\mathrm{C} 1+\mathrm{p}^{*} \mathrm{C} 2+\mathrm{q}^{*} \mathrm{C} 3=2 \mathrm{C}
$$

For 2 Proccssors:

$$
E[O R T]=C 1+\min \left(q^{*} C 3, p^{*} C 2\right)=(1+\min (p, 1-p) C
$$

For 3 Processors:

$\mathrm{E}[\mathrm{ORT}]=\mathrm{C}$ 


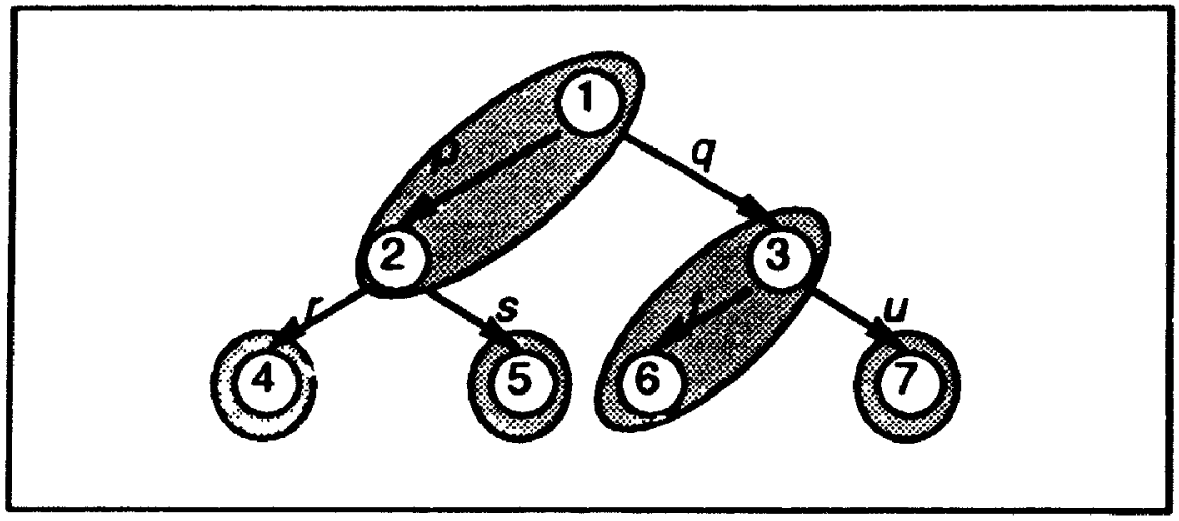

Figure 5.2 - Possible Request Groups for Dialog Graph when 2 Processors are Available. All node require same compute time and think times are zero.

Another interesting property of CCZT graphs is how the expected overall response time can he calculated in the case when the RGC algorithm is applied to find the optimal solution. If we assume the graph in Figure 5.2 is a CCZT with compute time of 1 . we find that the F[ORT] expression for when 2 processors are available is:

$$
E[O R T]=1+\min (\operatorname{pr}(1+\min (v, w))+q(1+\min (s+t, s+u, t+u)), 2 p+q))
$$

This equation assumes that we allocate processing time on a unit basis, where the unit is equal to the compule time of the focus. We assume that the allocation of a fraction of a unit of processing time will not result in a lower expected overall response time. While the proof of this statement appears obvious we have been unable to prove that it is actaully true. We assume for the remainder of this thesis that fractional unit allocations will not result in lower expected overall response times. Assuming only unit allocations we see that as we discussed in Chapter 4, the minimum expected overall response time for an uscr/interface dialog is equal to the minimum sum of the delays introduced by each node [eyuation 4.4.1]. In the case where the graph is CCZT, this is the same as minimizing one 
plus the sum of the relative probabilities of the paths that lead from the initial foxus to the head of each of the request groups for the dialog. or more precisely:

\section{Lemma 5.0}

$E[O R T]=C\left(1+\Sigma p_{R}\right)$,

Where $R=$ path from root group to descendant group whose ficus is not completely computed at the time that the user requests its result.

$$
\begin{aligned}
& P_{R}=\text { relative probability of visit to deseendent group } \\
& C=\text { the compute time. }
\end{aligned}
$$

\section{Proof}

Let $\mathrm{H}$ be the set of all nodes that are contained in a request group other then their own request group

Dn = Delay introduced by node $n$

$\mathrm{C}=$ compute time (constant for all nodes)

$F=$ initial focus

$\forall \mathrm{n} \in \mathrm{H}, \mathrm{D}_{\mathrm{n}}=\mathrm{C}$

$\forall \mathrm{n} \in \mathrm{H}, \mathrm{D}_{\mathrm{n}}=\mathbf{0}$

From [1] we see that if a node is only a member of its own request group then the node does not receive any eager computation before the user demands its result. Therefore the dealt introduced by the node is C. From [2] we sec that if a node appears in another request group other than its own, then the node is complealy 
computed (assuming that fraction allocations do not yield lower E[ORT] values then non-fractional allocations).

$$
\begin{aligned}
& \left.I:[O R T)=P_{\text {rel }_{0}}{ }^{* I}\right)_{0}+P_{\text {rel }_{1}}{ }^{* D_{1}}+\ldots+P_{\text {rel }_{n}} * D_{n} \\
& \text { [from equation 4.4.1] } \\
& =\Sigma P_{r e l} * D_{n} \ln \alpha \mathbf{x} \\
& {\left[D_{n}=C \text { for } n \boldsymbol{\alpha}: H\right]} \\
& =\sum P_{\mathrm{rel}} * C \ln \alpha \mathbf{H} \\
& =C^{*}\left(\Sigma P_{\text {rel }_{n}}\right) \ln \alpha \mathbf{H} \\
& =C^{*}\left(1+\Sigma P_{\mathrm{rel}_{n}}\right) \ln \text { \& } H \text { and } n=F \\
& \text { [Prel focus is 1] } \\
& {\left[\mathrm{E}[\mathrm{ORT}]=\mathrm{C}\left(1+\Sigma \mathrm{pR}_{\mathrm{R}}\right)\right.}
\end{aligned}
$$

Using Lemma 5.0, we will now examine scine interesting graphs whose request groups are independent of the probabilities assigned to the ares in the graph.

For some graphs we can easily determine the minimum expected overall response time without knowledge of the probabilities assigned to the individual arcs. We can determine the minimum expectud response time for a complete binary tree of depth 4 when 3 processors are used without knowledge of the probabilities assigned to the individual arcs. We can show that for this particular graph, for any assignment of probabilities to the arcs (where every arc has a probability greater than 0 ), the minimum expected overall response time is 2.0 . 


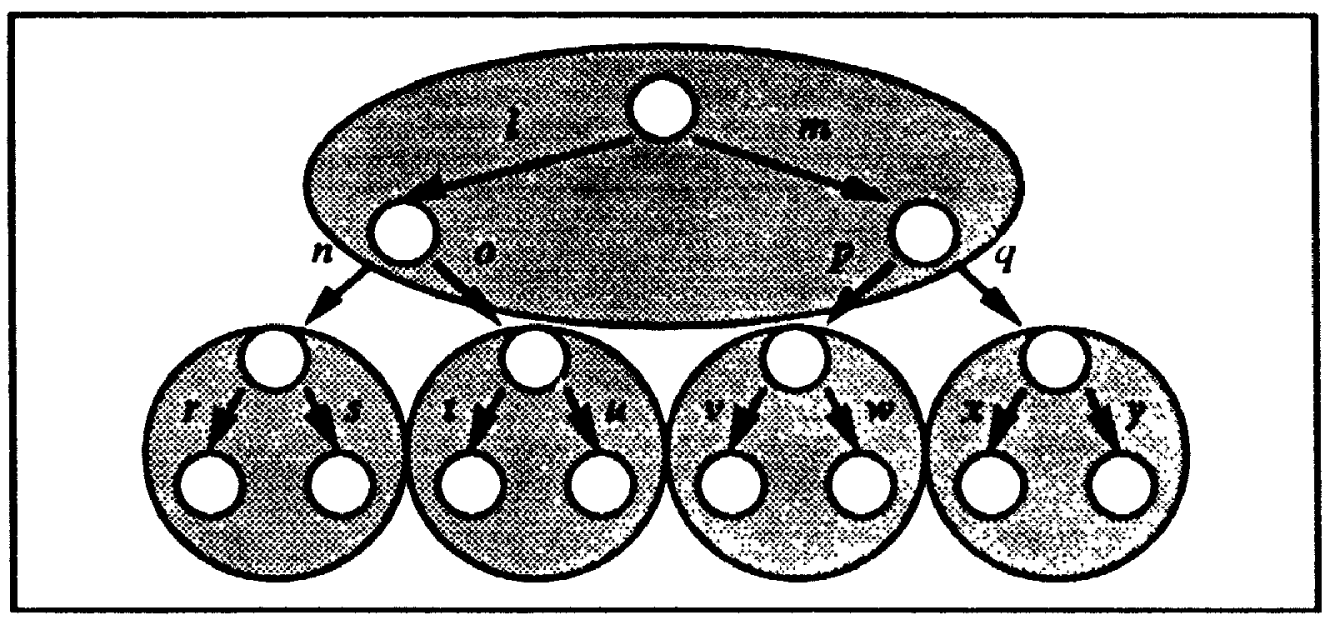

Figure 5.3 - Optimal Groups for a Complete Binary Tree of Depth 4 (Think Time 0, Compute Time Constant for all Nodes)

To see that the request groupings shown in Figure 5.3 are indeed optimal, we need only compare it with Figure 5.4. For the groups in Figure 5.3, the expected overall response time (assuming a constant compute time equal to 1) is:

$$
\begin{aligned}
\mathrm{E}[\mathrm{ORT}] & =1+\ln +l o+m p+m q & & {[n+o=1, p+q=1] } \\
& =1+l+m & & {[l+m=1] } \\
& =2 & &
\end{aligned}
$$

Now, consider Figure 5.4. We can immediately see that the expected overall computie lime imposed by this partial grouping is:

$$
\begin{aligned}
\mathbf{E}[\mathrm{ORT}] & =1+\ln r+\ln s+l o t+l o u+m+\mathrm{f}(m) & & \mid r+s=1, l+u=1\} \\
& =1+\ln +l o+m+f(m) & & |n+o=1| \\
& =1+l+m+\mathrm{f}(m) & & {[l+m=1] } \\
& =2+f(m) & &
\end{aligned}
$$




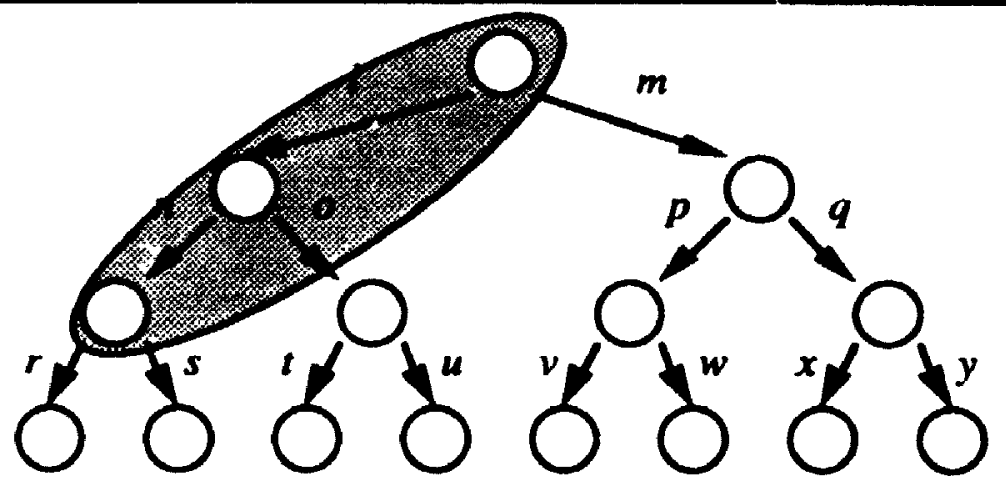

Figure 5.4 - Non-Optimal Partial Grouping for a Complete Binary Tree of Depth 4 (Think Time 0, Compute Time Constant for all Nodes

We use the $f(m)$ term to denote the value that will be contributed to the E[ORT] by the portion of the tree reachable by are $m$. Since all of the probabilities are greater than 0 (otherwise the arc and the tree that the are connects to could be pruned from the graph) and there are seven nodes reachable on paths including are $m$, the value of $f(m)$ must be nonzero. We can immediately see that the E[ORT] for these groupings would always be greater that the E[ORT] for Figure 5.3.

While it may appear that we could extend our analysis to construct a simple closed form solution for computing the expected overall response time for complete binary trees, consideration of a complete binary tree of depth 3 (Figure 5.5) when 3 processors are involved proves this to be false. Figure 5.5 illustrates that this is not so. Instead, we find that the approach used for the depth 4 tree results in the worst possible expected overall response time. For the groups in Figure 5.3, the expected overall response time (assuming a constant compute time equal to 1) is: 


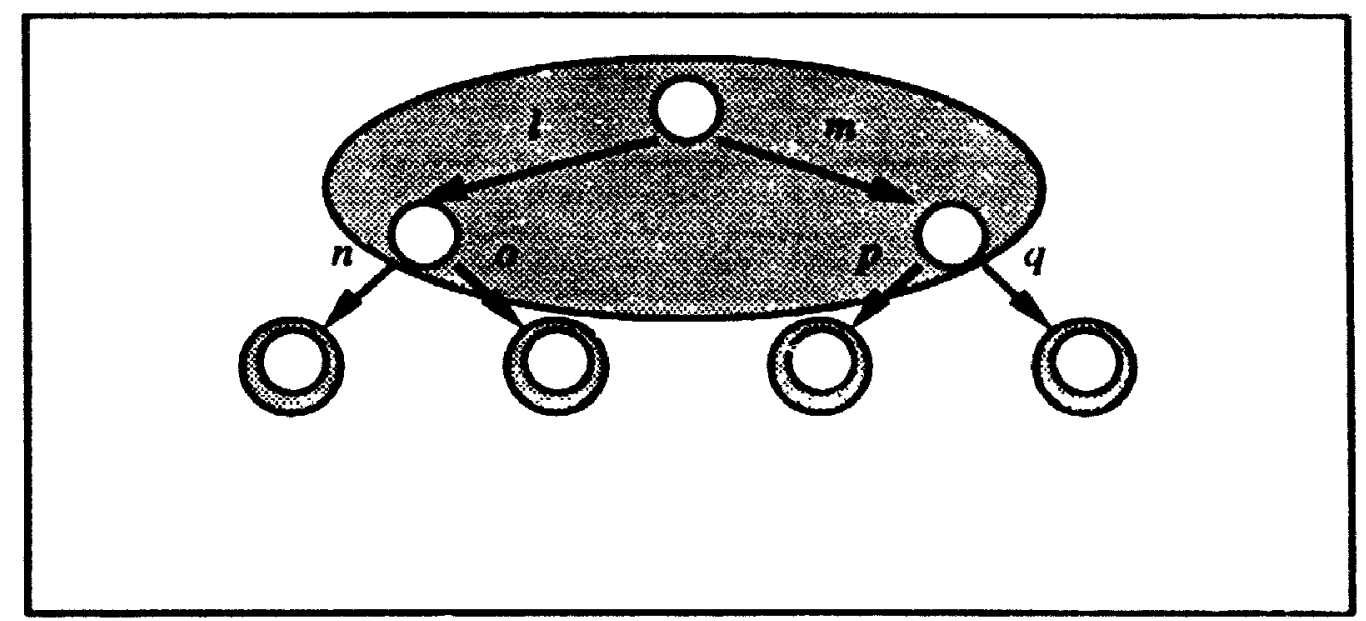

Figure 5.5 - Groups For A Complete Binary Tree f Depth 3 Using the Same Grouping Stratgey Employed on a Binary irec of Depth 4 (Think Time 0, Compute Time Constant For All Nodes)

$$
\begin{aligned}
\mathrm{E}[\mathrm{ORT}] & =1+\ln +l o+m p+m q & & \mid n+l)=1, p+y=1 \mid \\
& =1+l+m & & |l+m=1| \\
& =2 & &
\end{aligned}
$$

Now if we consider the groupings in Figure 5.6, we can see that the expected overall response time (assuming a constant compute time equal to 1) is:

$$
\mathrm{E}[\mathrm{ORT}]=1+l o+m
$$

Since we know that $l+m=1$ and that $o<1($ since $n>0$ and $n+o=1), l o+m<1$ (since $l o$ $<l)$. Therefore $1+l o+m$ is always less than 2.0 , so the groupings shown in Figure 5.5 are always the worst possible groupings.

The two binary tree examples and the opposing nature of their solutions helps to illustrate how not only the arc probabilities and graph structure can effect the minimum expecked 
overall response time, but so too can the depth of the graph and the number of available processors

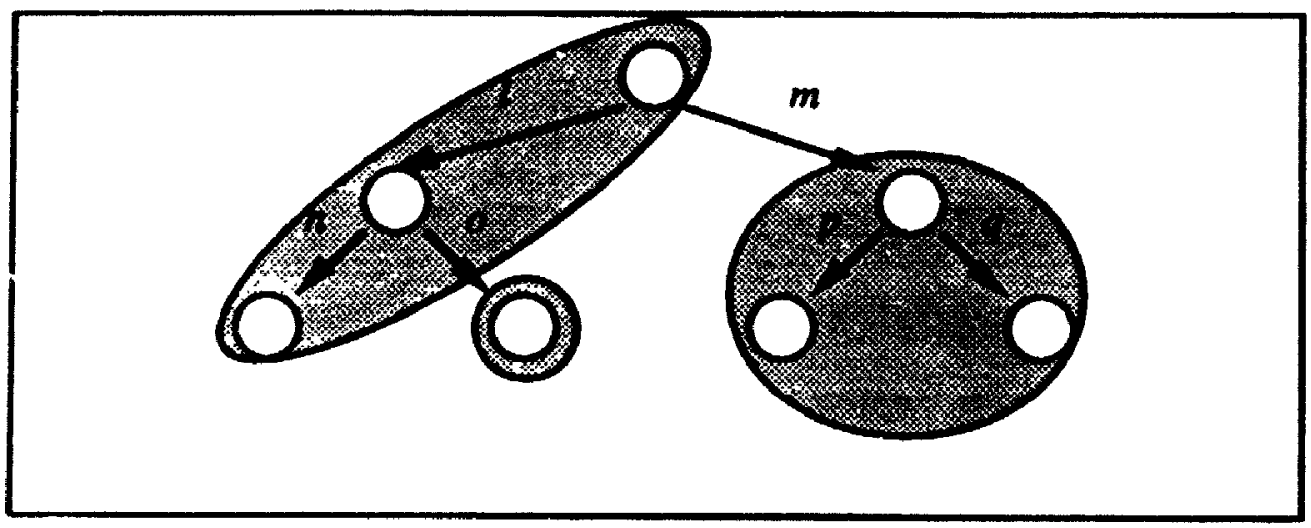

Figure 5.6 - Possible Groupings for A Complete Binary Tree Of Depth 3 (Think Time 0, Compute Time Constant For All Nodes

In this section we have discussed why the derivation of a general equation for the minimum overall expected response time for a dialog is so difficult. To illustrate why the derivation of a simple equation is so difficult, we have presented two examples that illustrate how some optimal groups are independent of the probabilities assigned to ares in the dialog graph and how the groupings that result in the minimum expected overall response time can be sensitive to the depth of the graph. 


\subsubsection{Deriving Heuristics}

As we have observed, the exponential nature of the RGC algorithm makes its application to dialog graphs of more than trivial complexity. a very time consuming endeavor. However. the analysis of the behavior of the RGC algorithm in cases where Lemma 5.1 applies give's rise to an interesting class of heuristics.

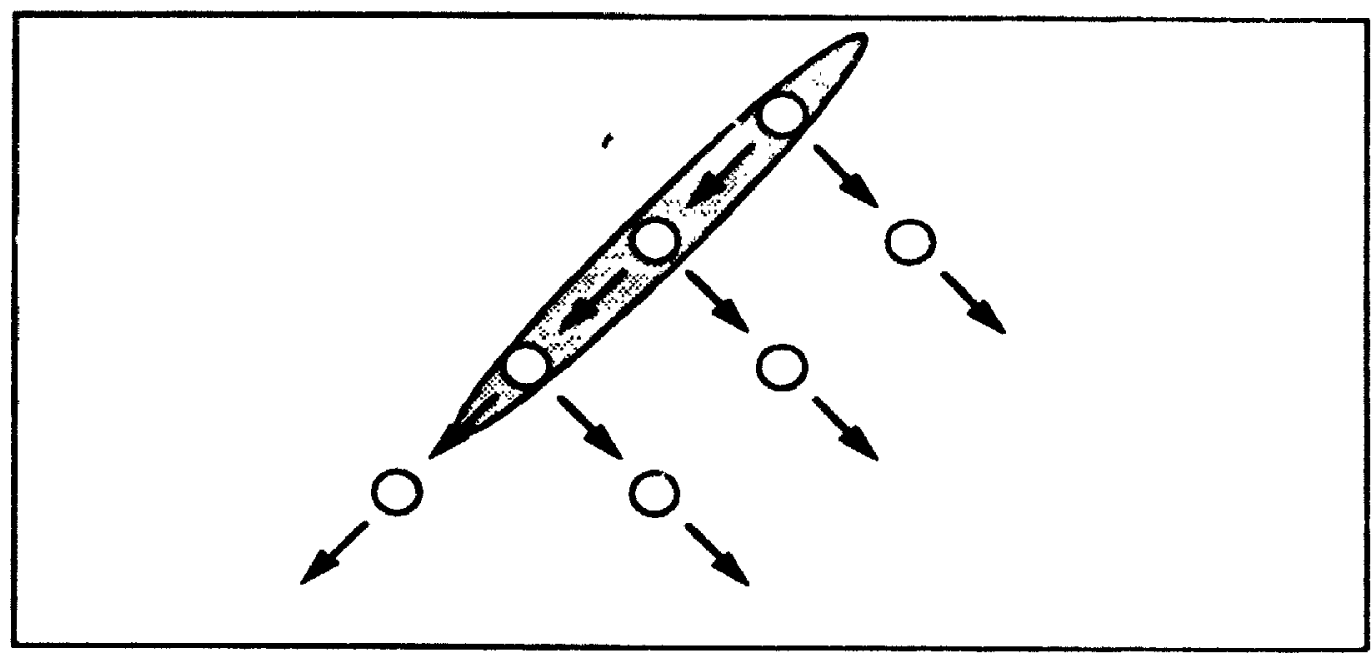

Figline 5.7 - Partial Grouping

As motivation in deriving heuristics we considered the partial grouping in Figure 5.4 and attempted to answer the y lestion of "If we had one more processor at our disposial, which node would we select to add to the request group to keep the overall expecked response time to a minimum?". Since we are only adding a member to the request group constructed using P processors to construct the request group for P-1 processsirs, we cannol hope to achieve with any regularity the construction of the same request groups that would tre produced by the RGC algorithm. As we have observed with the RGC algorithm, when the number of processors is increased the groupings constructed may not even clescly resemble those groupings constructed when fewer processors are involved. 

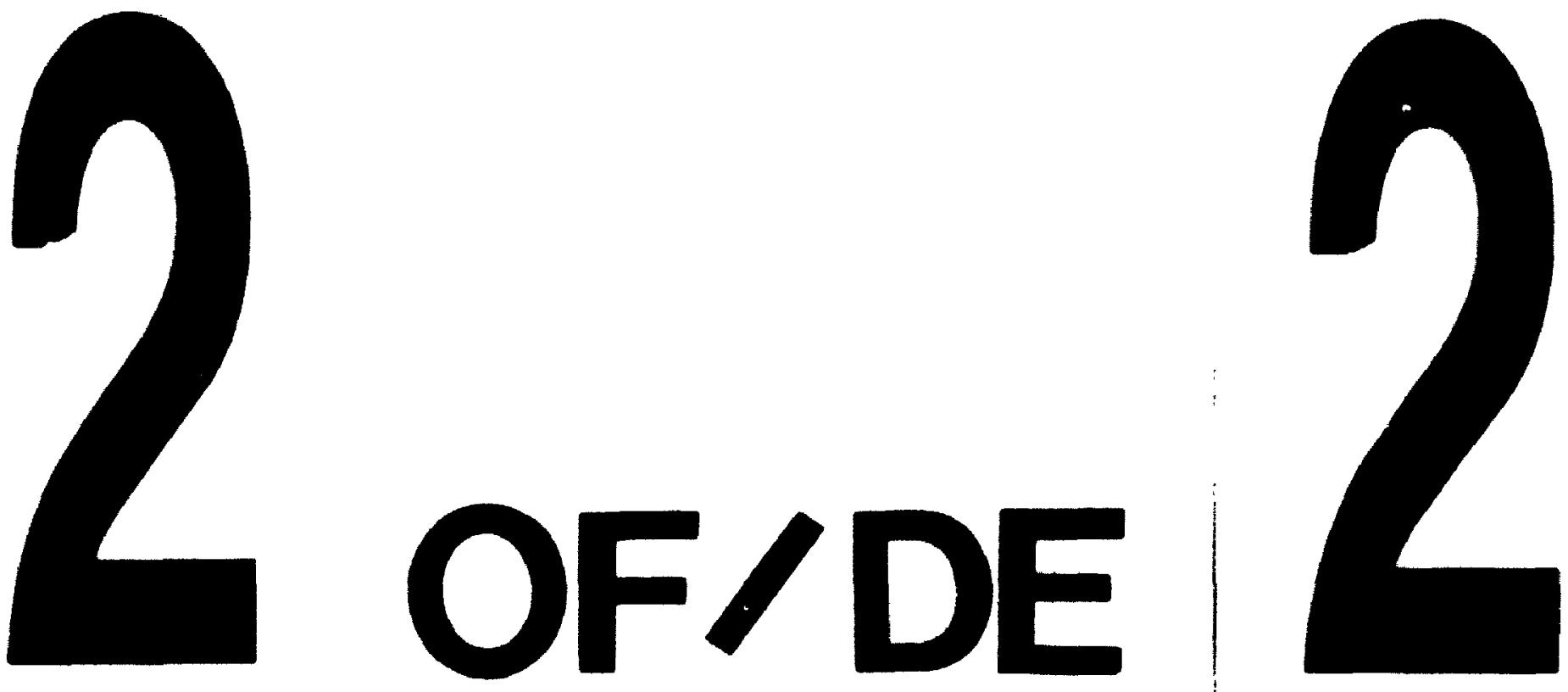

PM-1 3\%" "X4" PHOTOGAAPHIC MICROCOPY TARGET NOS 1010a AMSI/18O "2 EOUIVALENT

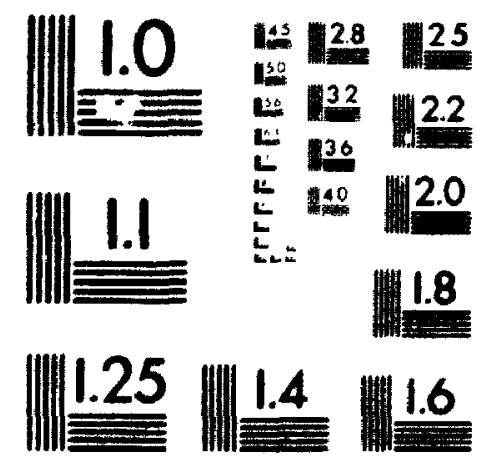


We found that since we are attempting to minimize the sum of the probabilities of the ares that connect the various request groups (from Lemma 5.0), we should select the node that is associated with the are with the highest relative probability. However, from our experiences with the RGC algorithm we have found that the selection of a node hased solely upon its probability is not sufficient to guarantce an optimal solution. To improve the accuracy of E[ORT] achieved by a heuristic we need some means by which to weight the probability of a request being demanded by 1) how the inclusion of the request in the current request group and 2) the amount of processing time consumed by the request. We could then select the node that represents the greatest potential reduction in the overall response time. This function can be written as:

For the nodes that can be directly connected to the current group, we add the node with $\max \left(\mathrm{P}_{\mathrm{i}}{ }^{*}\right.$ [Reduction in Cost if Node $\mathrm{i}$ Included])

We have experimented with 2 heuristics that use quite different reduction prediction functions. The first heuristic employs a reduction prediction function that always returns a constant regardless of the node that is evaluated. This has the net effect of favoring the nodes with the highest relative probabilities for inclusion into the current group. Since the heuristic includes those nodes with the highest relative probabilities in'o the current request group, it is minimizes the sum of the relative probabilities of the arcs emitting from the constructed grouping. We refer to this heuristic as the Select Most Probable Nodes heuristic. The second heuristic employs a reduction prediction function that returns BDepih(i), where B is the maximum branching factor for any node in the graph. Depth(i), returns the depth of the node relative to the position of the initial focus. This has the net effect of favoring the inclusion of the nodes that are on the most probable path emanating 
from the focus of the group. We refer to this heuristic as the Select Most Probable Nodes heuristic. We have chosen these two heuristics because they represent opposite extremes of reduction prediction functions. We have experimented with other heuristics in this class but we have not found one that consistently produces more accurate results.

We realize that heuristics that make their decisions based solely on local state information will not obtain results that closely approximate those of the RGC algorithm for all graphs. 1. Wer to obtain more accurate results the reduction prediction functions we will have to include more information about the global state and how the inclusion or exclusion of a node effects the overall state. A more accurate heuristic would employ information such as the maximum/minimum depth or the number of nodes in a sub-tree in constructing request groups (Recall from section 5.2.2 how the depth of the tree can effect the optimal groupings). One heuristic based on the use of the number of nodes in a sub-tree would attempt to select nodes so that the number of nodes in each remaining sub-tree is a multiple of the number of available processors. This heuristic would be most useful when all of the nodes have the same compute time and zero think times. By attempting to form the uncomputed sub-trees such that they can be computed with an even multiple of the number of available processors, the heuristic attempts to minimize the number of processors left idle when the number of uncomputed nodes around a leaf node is less than the number of processors.

In the following sections we discuss general forms of these heuristics that can be applied to any dialog graph in order to compute the request groups and overall expected response time. We also describe how we can compute the expected overall response time that each heuristic would yield for some restricted classes of graphs. We also discuss how we can estimate the speedup for these same types of graph. 


\subsection{Heuristics}

We examine both the empirical and analytical properties of the Select Most Probable Nodes and Select Most Probable Path heuristics relative to the algorithm and to each other.

\subsubsection{Heuristic 1 -- Select Most Probable Nodes}

This heuristic selects the most probable nodes that the focus will visit given the current Ireation of the focus. This has the effect of allocating any available sponsorship units to those nodes who will most likely become the focus soon after the location of the current focus changes. The heuristic performs the following steps to determine the groups for each. node:

1. Let SU be the number of sponsorship units that this node has to offer; GRP represent the request group which is being constructed for the node; MAX_SU be the maximum number of sponsorship units that this node can allocate to any member of its request group;

Initialize:

$$
\begin{aligned}
& \text { SU = Remaining Compute Time * Processors + Think Time * Processors; } \\
& \text { GRP = focus; } \\
& \text { MAX_SU = Compute Time + Think Time; }
\end{aligned}
$$

2. If $\mathrm{SU}$ is equal to zero then go to step 8. 
3. If all the nodes have been evaluated then go to step 8.

4. Get the node with the largest relative probability which has not been evaluated. Let $\mathrm{CN}$ be the node selected. Let CT equal the compute time required by that node to calculate the result of its request.

5. If the Compute Time required by the request is less than maximum amount that the node can receive and it is also less than number of sponsorship units remaining then

$\mathrm{GRP}=\mathrm{GRP}+(\mathrm{CN}, \mathrm{CT}) ; \mathrm{Si}=\mathrm{SU}-\mathrm{CT} ; \mathrm{CT}=0$

go to step 2.

6. If the Compute Time required by the request is less than maximum amount that the node can receive but it is greater than the number of sponsorship units remaining then

GRP = GRP + (CN, SU);CT $=\mathrm{CT}-\mathrm{SU} ; \mathrm{SU}=0$

go to step 8

Otherwise, if the Compute Time required by the request is greater than maximum amount that the node can receive and the number of sponsorship units remaining is less than the maximum amount that the node can receive $\mathrm{GRP}=\mathrm{GRP}+(\mathrm{CN}, \mathrm{SU}) ; \mathrm{CT}=\mathrm{CT}-\mathrm{SU} ; \quad \mathrm{SU}=0 ;$

go to step 8. 
7. If the Compute Time required by the request is greater than maximum amount that the rude can receive and the number of sponsorship units remaining is greater than the maximum amount that the node can receive

GRP $=$ GRP + (CN, MAX_SU $) ; C T=C T-M A X \_S U ; S U=S U$ MAX_SU;

go to step 2.

\section{8. retum GRP}

Using this heuristic, the following groupings would be generated for the dialog in Figure 5.0:

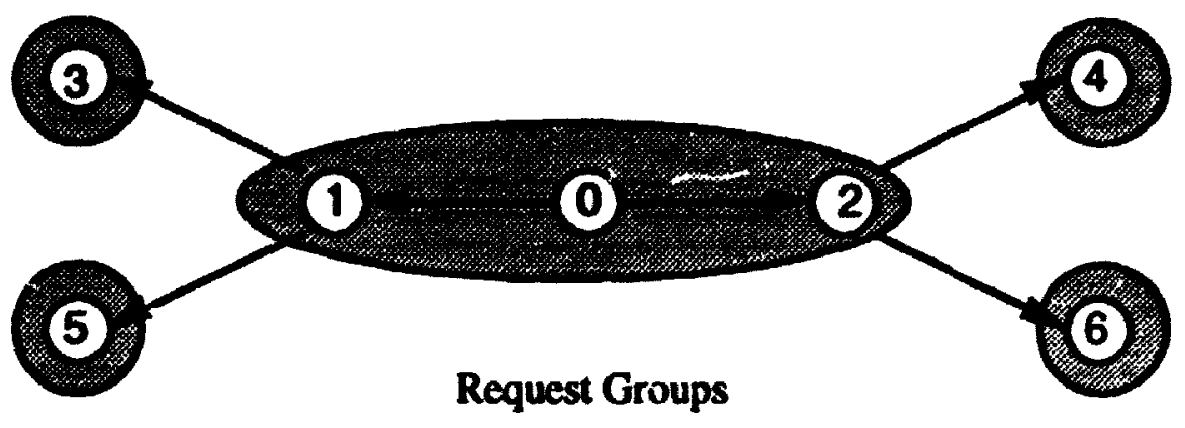

Nodes Groups

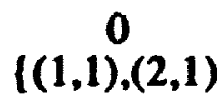

$\begin{array}{lllllll}1 & 2 & 3 & 4 & 5 & 6 & \text { E[ORT] } \\ \text { (1) } & 1\} & \text { (1) } & \text { (1) } & \text { (1) } & \text { (1) } & 2.0\end{array}$




\begin{tabular}{|c|c|c|c|c|c|}
\hline \multirow[b]{3}{*}{$\begin{array}{l}\text { Number } \\
\text { of Nodes } \\
\text { in Graph }\end{array}$} & \multirow[b]{3}{*}{$\begin{array}{l}\text { Maximum } \\
\text { Branching } \\
\text { Factor }\end{array}$} & \multicolumn{4}{|c|}{ Comparison of E[ORT] Values } \\
\hline & & \multicolumn{2}{|c|}{8 Processors } & \multicolumn{2}{|c|}{16 Processors } \\
\hline & & $\begin{array}{l}\text { Relative } \\
\text { Error } \\
\text { (in \%) }\end{array}$ & $\begin{array}{l}\text { Absolute } \\
\text { Difference }\end{array}$ & $\begin{array}{l}\text { Relative } \\
\text { Error } \\
\text { (in \%) }\end{array}$ & $\begin{array}{l}\text { Absolute } \\
\text { Difference }\end{array}$ \\
\hline \multirow{4}{*}{$\begin{array}{l}200- \\
220\end{array}$} & 2 & 6.43 & 0.165 & 6.80 & 0.125 \\
\hline & 4 & 5.97 & 0.167 & 8.11 & 0.166 \\
\hline & 6 & 5.56 & 0.171 & 7.40 & 0.159 \\
\hline & 8 & 5.45 & 0.188 & 6.72 & 0.165 \\
\hline \multirow{4}{*}{$\begin{array}{l}400- \\
420\end{array}$} & 2 & 5.97 & 0.123 & 8.70 & 0.147 \\
\hline & 4 & 5.38 & 0.122 & 6.31 & 0.116 \\
\hline & 6 & 4.70 & 0.118 & 5.83 & 0.118 \\
\hline & 8 & 4.69 & 0.122 & 6.77 & 0.143 \\
\hline \multirow{4}{*}{$\begin{array}{l}800- \\
820\end{array}$} & 2 & 5.93 & 0.117 & Algorithm & Agorithm \\
\hline & 4 & 5.69 & 0.121 & $\begin{array}{l}\text { Falled } \\
\text { To Arrive }\end{array}$ & $\begin{array}{l}\text { Fauled } \\
\text { To Arrive }\end{array}$ \\
\hline & 6 & 4.60 & 0.106 & At & At \\
\hline & 8 & 4.96 & 0.124 & & Result \\
\hline \multirow{4}{*}{$\begin{array}{l}1600- \\
1620\end{array}$} & 2 & 4.68 & 0.087 & Algorithm & Algorithm \\
\hline & 4 & 5.96 & 0.124 & $\begin{array}{l}\text { Failed } \\
\text { To Arrive }\end{array}$ & $\begin{array}{l}\text { Falled } \\
\text { To Arrive }\end{array}$ \\
\hline & 6 & 5.74 & 0.131 & & Dis \\
\hline & 8 & 5.87 & 0.142 & & \\
\hline
\end{tabular}

Table 5.0 - Comparion of Results of Select Most Probable Nodes Heuristic to the Results of the RGC Algorithm. Relative Error based on (Min E[ORT] - Heurictistic E[ORT])Min E[ORT]

This heuristic is attractive because the amount of analysis performed is constant for a graph regardless of the number of processors involved. The effort of determining which node is the most probable of the unevaluated nodes is $O(N \log )$ (where $N$ is the number of nodes 
in the graph). These two factors make this method very attractive from an efficiency standpoint.

Table 5.0 summarizes the accuracy results we have gathered for this heuristic. The table lists the relative and absolute error for the E[ORT] for the groups generated by the heuristics versus those generated by the RGC algorithm. We could not obtain E[ORT] values for the RGC algorithm for graphs containing 800-820 nodes and 1600-1620 nodes because the RGC algorithm ran out of memory. We have varied the size of the graph, the number of descendants per node and the number of processors involved in an attempt to gain an accurate understanding of how the heuristic will perform. For each of the combinations of these attributes, we applied both the algorithm and the heuristic to the same collection of 20 random graphs. For each random graph we randomly assigned probabilities between 0.1 and 1.0 to each arc, and we enforced that the sum of the probabilities of the arc emanating from a node had to equal 1.0. The number of descendants per node varies between 0 for a leaf node and up to the maximum branching factor for the graph being analyzed. Each node had a compute time of 1.0 and a think time of $\mathbf{0}$.

The most prominent feature of the Select Most Probable Nodes heuristi: is the consistency of the low relative error it produces for a given number of processors, regardless of the graph. This is a good indication that a reduction prediction function used to approximate the RGC algorithm should definitely perform some form of breadth first search based on selecting nodes that have a high relative probability. In section 5.4.1 we discuss the results for the Select Most Probable Path heuristic and how they compare. 
Another prominent feature of the heuristic is that as the number of processors increase the relative error increases quite slowly. An increase was to be expected since the more processors involved means that the heuristic will have a greater chance of constructing request groups that do not match those produced by the RGC algorithm.

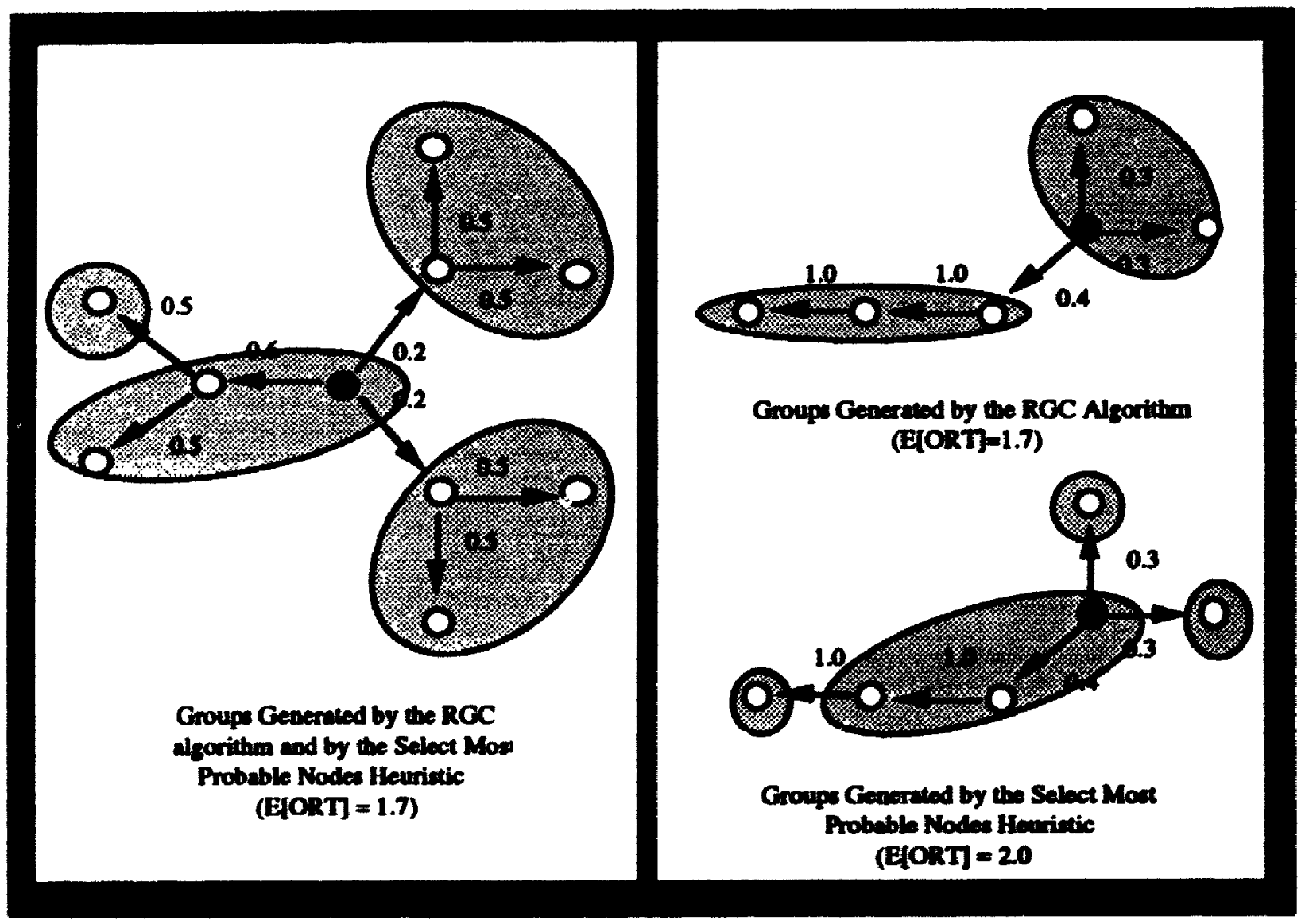

Figure 5.8 - Two graphs illustrating the poseible descrepencies between the groupe generented by the RCC Algorithen and the Select Moat Probeble Nodes Heurietic

The major deficiency of the Select Most Probable Node heuristic, is that it only considers the impact of the request group with respect to locally minimizing the response time and not the impact the request group has on the minimization of overall response time. For this reason, the heuristic can create groupings that result in expected overall response time which are much higher than those generated by the RCC algorithm. Figure 5.8 shows two 
example dialog graphs. In one case the Select Most Probable Nodes heuristic and the RGC algorithm compute the same groupings. In the other, the groups generated are quite different and result in the expected overall response time predicted by the heuristic being tnuch higher than that predicted by the RGC algorithm. This illustrates how the reliance of the Select Most Probable Nodes heuristic (and ultimately any heuristics of the same class) on solely local state information makes it vunerable to constructing request groups that can result in expected overall response simes that deviate greatly from those produced by the RGC algorithm

\subsubsection{Heuristic 1 -. Analysis}

The request groups generated by the Select Most Probable Nodes heuristic can also be identical to those generated by the RGC algorithm. We can see for the graph in Figure 5.2, that if the two ares directly emanating frum a node have a higher relative probability than the arcs emanating from the node's descendants (i.e., $m>(l n$ and $l o$ ) and $l>(m p$ or $m q$ )) the request groups generated by the heuristic would be identical to those generated by the RGC algorithm. We can also see that if the relative probabilities are not such that the directly connected arcs are the most probable, the heuristic will not construct the optimal grouping. This divergence of the heuristic from the RGC algorithm is a result of the heuristics inability to account for the global impact of node selections on the overall expected response time.

As we did with the RGC algorithm, we can also compute the expected overall response times that would result from the request groups produced by this heuristic. The general equation for the expected overall response time for a graph can be quite cumbersome since it must account for all of the different possible groups that could be constructed based on 
the relative probabilities of each of the nodes. However, the complexity of the resulting equation is usually considerably less than the RGC algorithm's equation for the same graph. If we restrict our attention to CCZT graphs where all nodes have the same branching factor with the $i^{\text {th }}$ arc emanating from each node having the same probability, we can find a recurrence relation for the value of E[ORT].

$$
\begin{aligned}
& \text { [equation 5.2.2.1] } \\
& \text { Where a }=\text { path from root node to descendant group and } \\
& \qquad \begin{array}{l}
p_{a}=\text { relative probability of visit to descendant group } \\
d(a) \text { returns the depth of the descendart group relative to the } \\
\text { focus of the root group. }
\end{array}
\end{aligned}
$$

$$
E\left[O R T_{D}\right]=0, \text { for } D<0
$$

For the trivial case of a complete $k$-ary tree of depth $d$, where each node has an absolute probability of $1 / \mathrm{k}$ being visited, the recurrence relation reduces to:

$$
\begin{aligned}
& E\left[O R T_{D}\right]=1+\left(P-\left(\left(k^{d}-1\right) /(k-1)\right)\right)^{*}(1 / k)^{d * E}\left[O R T_{D-d-1}\right]+ \\
& \left.\left.\left.\left(k^{d+1}-1\right) /(k-1)\right)-P\right)^{*}(1 / k)^{d * E[O R T} D_{-d}\right] \\
& \text { where } P=\text { Number of Processors, } d=\text { floor }(\operatorname{logk}(P(k-1))) \\
& E[O R T D]=0 \text {, for } D<0
\end{aligned}
$$

This equation simply states that the expected overall response time for a complete k-ary tree of depth $D$ is equal to the sum of the compute time associated with the focus of the group [the constant 1 term], the number of nodes in the request group on lowest occupied level that are in the request group (number of nodes at depth d) multiplied by the relative 
probability that the node will become a focus $((1 / k) d)$ [the second term], and the numbr of nodes on the lowest occupied level that are not in the request group multiplied by the probability that the node will become a focus $\left((1 / \mathrm{k})^{\mathrm{d}}\right)$ [the third term].

In section 5.2.2 we noted how the optimal groupings and hence the E[ORT] for a dialog changes as the depth of the dialog graph increases. The E[ORTD] formula that we have derived above allows us to easily compare the performance of the Select Most Probable Nodes heuristic to the RGC algorithm as the depth of the dialog graph increases. Consider the graph in Figure 5.9.

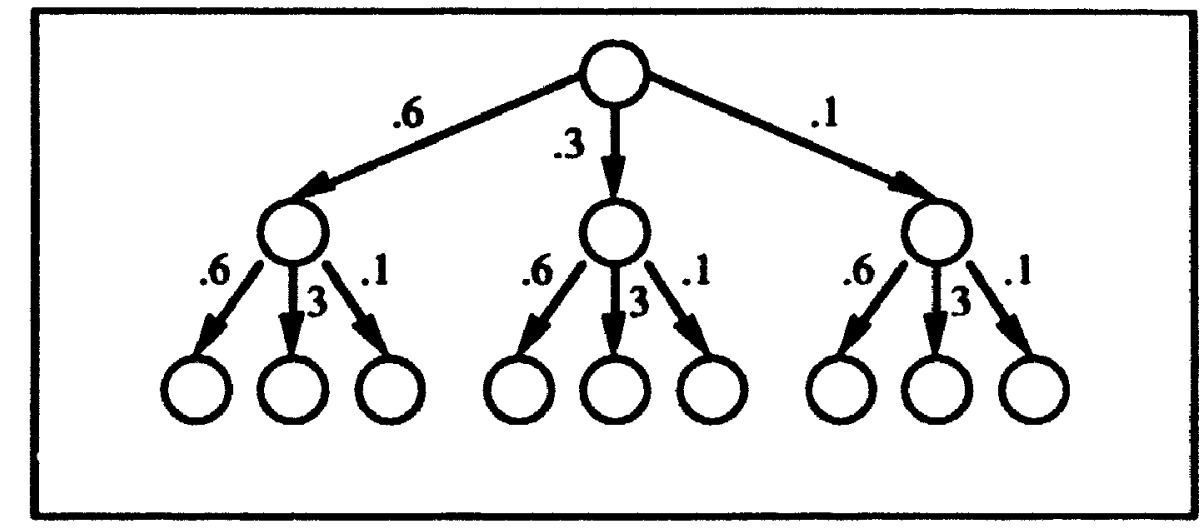

Figure 5.9 - 3-ary Tree used to Demonstrate how E[ORT] is affected by Depth

If we consider the case where we are allocating four processors, we can use the $E\left[O R T_{D}\right]$ formula to compute the expected overall response time resulting from the heuristic. We find that

$$
\begin{aligned}
E\left[O R T_{2}\right]= & 1.0+0.18 E\left[O R T_{0}\right]+0.06 E\left[O R T_{0}\right]+.18 E\left[O R T_{0}\right]+0.09 E\left[O R T_{0}\right]+ \\
& 0.03 E\left[O R T_{0}\right]+0.1 E\left[O R T_{1}\right]
\end{aligned}
$$$$
\mathrm{E}\left[\mathrm{ORT} \mathrm{T}_{1}\right]=1.0
$$ 
$\left.\mathrm{E}_{[\mathrm{ORT}}\right]=1.0$

Therefore $E\left[O R T_{2}\right]=1.64$

$\left(E\left[O R T_{2}\right]=1.46\right.$ from application of RGC algorithm). If we increase the depth of the troe to be four and perform our calculations again, we find that $E\left[O R T_{3}\right]=2.064\left(E\left[O R T_{3}\right]=\right.$ 1.968 from application of RGC algorithm). As we continue to increase the depth of the tree, we will observe that the change in the E[ORT] from depth $D$ to depth $D+1$ becomes smaller the further we move from the focus. Computation of E[ORT] using the formula is also useful if we have a graph with a uniform structure and homogeneous probabilities and we need to quickly decide which heuristic would be better suited to the graph.

While the recurrence relation for the E[ORT] ([equation 5.2.2.1]) for the request groups at first may not appear to be very interesting, we can manipulate this formula to determine a closed formula for the expected speedup that we can achieve using $P$ processors as opposed to a single processor to compute a $\mathbf{k}$-ary tree dialog graph. We have found the manipulation of this formula very useful in Chapter 7 for estimating the amount of speedup that we should expect to achieve in the worst case, when we consider graphs whose arc probabilities are random.

The heuristic performs a breadth-first allocation of processors. If we allocate $P$ processors to the solution of the graph then we know that a request group will have $\left(P-\left(k^{d}-1\right) /(k-1)\right.$ nodes from kevel $\mathrm{d}$. Since we know that the total number of nodes on level $\mathrm{d}$ is $\mathrm{k}^{\mathrm{d}}$, we can say that $\left(P-\left(k^{d}-1\right)(k-1)\right) / k^{d}$ percent of the time we will have a speedup of $d+1$. The other $1-\left(P-\left(k^{d}-1\right) /(k-1)\right) / k^{d}$ percent of the time we will have a speedup of $d$.

$$
E\left[S_{d}\right]=(d+1)^{*}\left(P-\left(k^{d}-1\right) /(k-1)\right) / k^{d}+d^{*}\left(1-\left(P-\left(k^{d}-1\right) /(k-1)\right) / k^{d}\right) \text {, [equation 5.2.2.2] }
$$


where $P=$ Number of Processors, $d=$ foor $\left(\log _{k}(P(k-1))\right)$

The Select Most Prc jable Nodes heuristic produces request groups that result in expected overall response time approaching those created by the RGC algorithm. An interesting property of the heuristic is that since it allocates processors in a breadth first manner, we can expect to waste a large amount of the computations that we perform (since only one approach of the "breadth" approaches is taken). The exact impact of the waste is most obvious when we consider a complete $k$-ary tree when all nodes have the same probability. Given that we already have a speedup of $S$, if we want to realize a speedup of $S+1$, we have to allocate $k^{S}$ more processors. Of these processors only one of the $k^{S}$ computations performed will be relevant, the other $k^{S}$ - 1 processors $u$ ill have wasted their computational effort. The diminishing returns created from the exponential growth of the number of processors required to achieve a speedup of $\mathrm{S}+1$ limits the size of the speedup we can achieve in practice. We can reduce the potential speedup by refining the probabilities we assign to each node to better reflect the choices the user will make. While this may not be practical in all instance, the identification of a highly dominant path in the graph, can help to achieve much better speedups. Researchers [Witte] have achieved a spoedup of $\mathrm{P} / 2$ when a highly dominant path is present. We present results that suggest similar speedup figures in section 7.4.

\subsubsection{Heuristic 2 .- Most Probable Path}

This heuristic constructs request groups for a node by following the most probable path that emanates from the focus node. This has the effect of improving the response time for 
those sequences of requests which are most common. The heuristic performs the following steps to determine the groups for each node:

1. Let SU be the number of sponsorship units that this node has to offer;

GRP represent the request group which is being constructed for the node;

MAX_SU be the maximum number of sponsorship units that this node can allocate to any member of its request group;

ACTIVE be the node which is currently being inspected;

TRAVERSED be a stack which contain those nodes which contains those nodes which have been traversed to get to the currently active node;

Initialize:

$$
\begin{aligned}
& \text { SU = Remaining Compute Time * Processors + Think Time * Processors } \\
& \text { GRP = focus } \\
& \text { MAX_SU = Compute Time + Think Time } \\
& \text { ACTIVE = node the request group is being constructed for } \\
& \text { TRAVERSED = nil }
\end{aligned}
$$

2. If $\mathrm{SU}$ is equal to zero then go to step $\mathbf{1 0 .}$

3. If ACTIVE is nil then go to step 10.

4. Get the node with highest absolute probability that is a direct relaion of ACTIVE but is not a memher of GRP. 
5. If all of the nodes which are directly reachable from ACTIVE have already been included in GRP then move back up the path to sce if any predecessors have direct relations which have not yet been included in GRP.

ACTIVE = pop TRAVERSED stack;

go to 3;

6. Extend the path to include the new node

push ACTTVE onto TRAVERSED stack;

ACTIVE = new node;

7. Let CT equal the compute time required by ACTIVE to calculate the result of its request.

8. If the Compute Time required by the request is less than maximum amount that the node can receive and it is also less than number of sponsorship units remaining then

$\mathrm{GRP}=\mathrm{GRP}+(A C T I V E, C T) ; C T=0 ; S U=S U-C T ; C T=0 ;$

go to step 2.

Otherwise, if the Compute Time required by the request is greater than maximum amount that the node can receive and the number of sponcorship units remaining is greater than the maximum amount that the node can receive

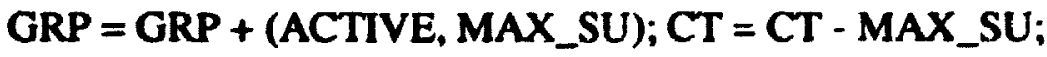

SU = SU - MAX_SU;

go to step 2. 
9. If the Compute Time required by the request is less thai. maximum amount that the node can receive but it is greater than the number of sponsorship units remaining then

GRP = GRP + (ACTIVE, SU $) ; C T=C T-S U ; S U=0$

go to step 10.

Otherwise, if the Compute Time required by the request is greater than maximum amount that the node can receive and the number of sponsorship units remaining is less than the maximum amount that the node can receive GRP = GRP + (ACTIVE, SU); CT =CT - SU; SU =0;

go to step 10 .

10. return GRP

Using this algorithm, the following groupings would be generated for the dialog in Figure 5.0:

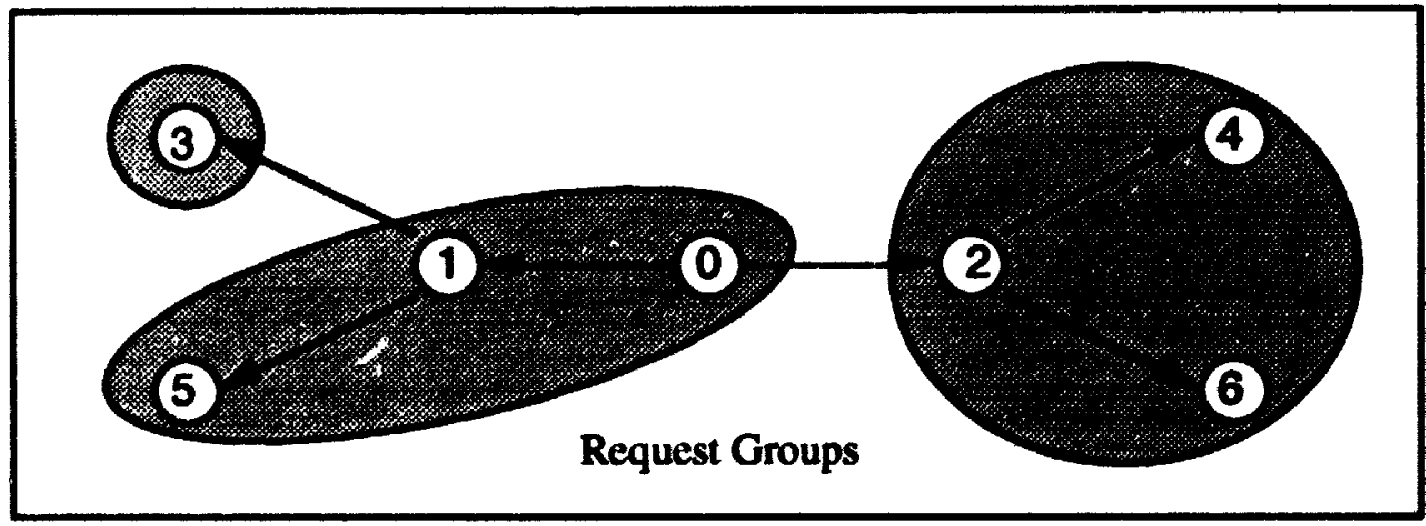

Nodes Groups
$\stackrel{0}{0}(1,1),(5,1)\}$
$\begin{array}{lc}1 & 2 \\ \text { ( ) } & \{(4,1),(6,1)\}\end{array}$
$\begin{array}{ll}3 & 4 \\ 11 & 11\end{array}$
5
6 E[ORT]
() 1.64 
The effort of determining which child node is the most probable of an unevaluated node is $O(M \log M)$ (where $M$ is the number of descendants of the node).

Table 5.1 summarizes the accuracy results we have gathered for this heuristic. The table lists the relative and absolute error for the E[ORT] for the groups generated by the heuristics versus those generated by the RGC algorithm. We could not obtain E[ORT] values for the RGC algorithm for graphs containing 800-820 nodes and $16(0)-1620$ nodes because the RGC algorithm ran out of memory. The graphs that we analyred are exactly the same as those analyzed using the Select Most Probable Node heuristic.

The results for the Select Most Probable Path Heuristic are not surprising. considering that when we discussed the results of the Select Most Probable Nodes Heuristic, we identified that the reduction prediction function for the RGC algorithm has a hreadth first uendency. We can see from Table 5.1, that the only time that the Select Most Probable Path heuristic produces results whose relative error is close to that of the Select Most Probable Nodes heuristic is when both the number of processors and the number of nodes in the graph are small. In these instances, the groups created by the heuristic are close to those created by the Select Most Probable Node heuristic. 


\begin{tabular}{|c|c|c|c|c|c|}
\hline \multirow[b]{3}{*}{$\begin{array}{l}\text { Number } \\
\text { of Nodes } \\
\text { in Graph }\end{array}$} & \multirow[b]{3}{*}{$\begin{array}{c}\text { Maximum } \\
\text { Branching } \\
\text { Factor }\end{array}$} & \multicolumn{4}{|c|}{ Comparison of E[ORT] Values } \\
\hline & & \multicolumn{2}{|c|}{8 Processors } & \multicolumn{2}{|c|}{16 Processors } \\
\hline & & $\begin{array}{c}\text { Relative } \\
\text { Error } \\
\text { (in \%) }\end{array}$ & $\begin{array}{l}\text { Absolute } \\
\text { Difference }\end{array}$ & $\begin{array}{c}\text { Relative } \\
\text { Error } \\
\text { (in \%) }\end{array}$ & $\begin{array}{l}\text { Absolute } \\
\text { Difference }\end{array}$ \\
\hline \multirow{4}{*}{$\begin{array}{l}200 \\
220\end{array}$} & 2 & 6.76 & 0.171 & 10.48 & 0.200 \\
\hline & 4 & 5.97 & 0.166 & 11.30 & 0.231 \\
\hline & 6 & 5.14 & 0.157 & 10.49 & 0.233 \\
\hline & 8 & 5.90 & 0.204 & 13.84 & 0.342 \\
\hline \multirow{4}{*}{$\begin{array}{l}400- \\
420\end{array}$} & 2 & 8.54 & 0.177 & 14.53 & 0.246 \\
\hline & 4 & 10.95 & 0.250 & 18.14 & 0.336 \\
\hline & 6 & 12.17 & 0.303 & 20.59 & 0.418 \\
\hline & 8 & 12.92 & 0.339 & 24.00 & 0.509 \\
\hline \multirow{4}{*}{$\begin{array}{l}800 \\
820\end{array}$} & 2 & 8.92 & 0.178 & Algorithm & Algorithm \\
\hline & 4 & 11.98 & 0.259 & Failed & $\begin{array}{c}\text { Failed } \\
\text { Tn Amive }\end{array}$ \\
\hline & 6 & 14.62 & 0.341 & At & Al \\
\hline & 8 & 14.16 & 0.355 & Result & Result \\
\hline \multirow{4}{*}{$\begin{array}{l}1600 \\
1620\end{array}$} & 2 & 13.06 & 0.253 & Agorithm & Algorithm \\
\hline & 4 & 15.07 & 0.314 & $\begin{array}{l}\text { Failed } \\
\text { To Arrive }\end{array}$ & $\begin{array}{l}\text { Failed } \\
\text { To Arrive }\end{array}$ \\
\hline & 6 & 16.08 & 0.367 & -80 & -80 \\
\hline & 8 & 14.68 & 0.358 & & \\
\hline
\end{tabular}

Table 5.1 - Comparion of Results of Select Most Probable Path Heuristic to the Results of the RGC Algorithm. Relative Error based on (Min E[ORT] - Heurictistic E[ORT])MMin E[ORT]

When the graphs become larger and/or the number of processors increases, so too does the error of the solution generated by the heuristic. The reason for the poor performance of the heuristic is clear: the larger the number of processors, the longer the path. The longer the 
path is, the less likely that the user will reach the end of the path before they select an "unexpected" node.

\subsubsection{Heuristic 2 -- Analysis}

As we did for the Select Most Probable Nodes heuristic in section 5.2, for some restricted classes of graphs, we can easily compute the expected overall response time that will result from the application of this heuristic. Consider the granh in Figure 5.2; the expression for expected overall response time using three processors is (assuming that the left-most arc is always the most probable and the compute time constant is 1):

$$
\begin{aligned}
\text { E[ORT }] & =1+l(n(r+s)+o)+m(1+p w+q) & & {[r+i=1] } \\
& =1+l n+l o+m+m p w+m q & & {[n+o=1, l+m=1] } \\
& =2+m p w+m q & & {[q=1-p] } \\
& =2+m(1-p v) & &
\end{aligned}
$$

Recall from section 5.2.1, that we found that the RGC algorithm (and again in section 5.3.4 with the Select Most Probable Nodes Heuristic) that the minimum E[ORT] is 2.0 for this graph. The Select Most Probable Path heuristic predicts an expected overall response time that is a factor of $m(1-p v)$ higher. In the best case (from an accuracy stand-point) this factor would be 0 , in the worst case (all probabilities approximately 0.5 ) it would be $\left(0.5^{3}+0.5^{2}=0.375\right)$. The result is a relative error of $18.75 \%$.

However, before we dismiss this heuristic as unacceptable, consider the case of the complete binary tree of depth 3. The depth-first approach of this heuristic will compute the 
exact same groups as the RGC algorithm. For this same graph, the Select Most Probable Nodes heuristic will be inferior because of its breadth-first search.

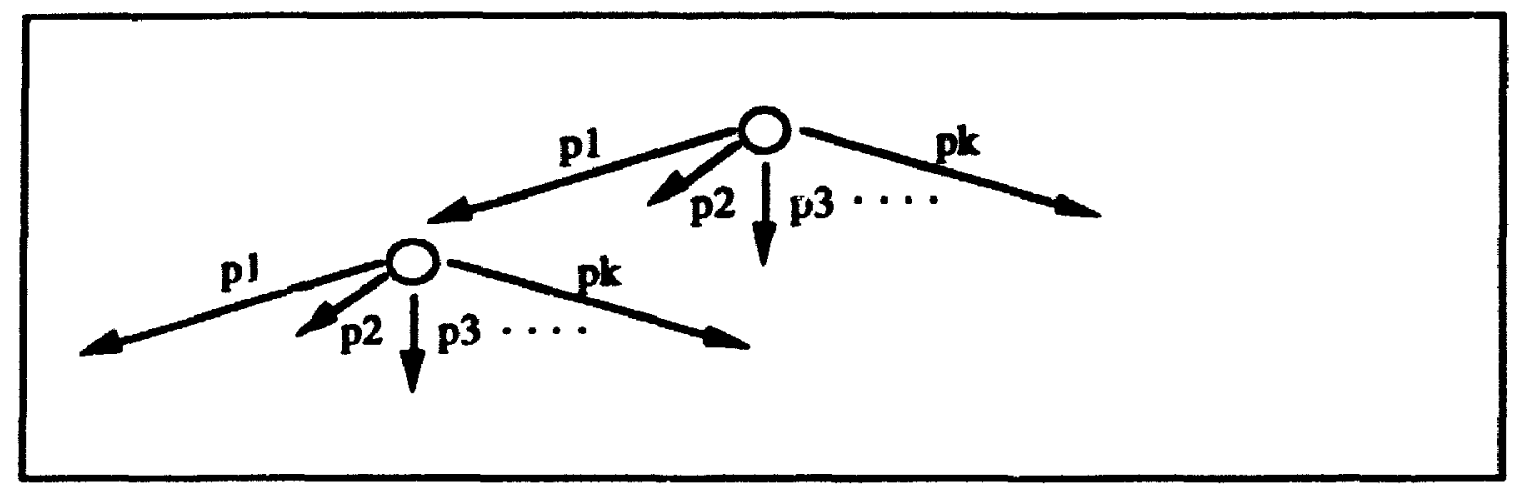

Figure 5.10 - A $k$-ary tree with the ith Arc Emanating from Each Node having the Same Probability

The fact that the Select Most Probable Path heuristic only considers the ancs emanating from a single node when it selects the next node to add to the request group, makes the E[ORT] recurrence relations much easier to derive than for the Select Most Probable Nodes heuristic. While the derivation of a formula to handle any tree of arbitrary structure is still complex, we can easily derive a relation for a complete $k$-ary tree that has the $\mathrm{i}^{\text {th }}$ arc emanating from each node having the same probability (as in Figure 5.10, where we assume that $p_{i}>p_{i+1}$ for all $\left.i\right)$.

$$
\begin{aligned}
& \left.E\left[O R T_{D}\right]=1+p_{1}{ }^{P *} E\left[O R T_{D-P}\right]+\sum_{i=1}^{P} \underset{p_{1}-1}{\sum} \sum_{\left(p_{j}\right.}^{k}{ }_{j=2}^{*}\left[O R T_{D-i}\right)\right), \\
& \text { where } P \text { is the number of processors and assuming } P<D \\
& E\left[O R T_{D}\right]=0 \text {, for } D<0
\end{aligned}
$$

This equation simply states that the expected overall response time for a complete k-ary tree of depth $D$ is equal to the sum of the compute times associated with the focus of the group [the constant 1 term], the overall expected response time for the node that would have been 
included in the path if we had one more processor to allocate [the second term], and the overall expected response times for each of the branches not taken weighted by the probability that they will be taken [the third term]. Figure 5.11 illustrates the derivation of the recurrence relation.

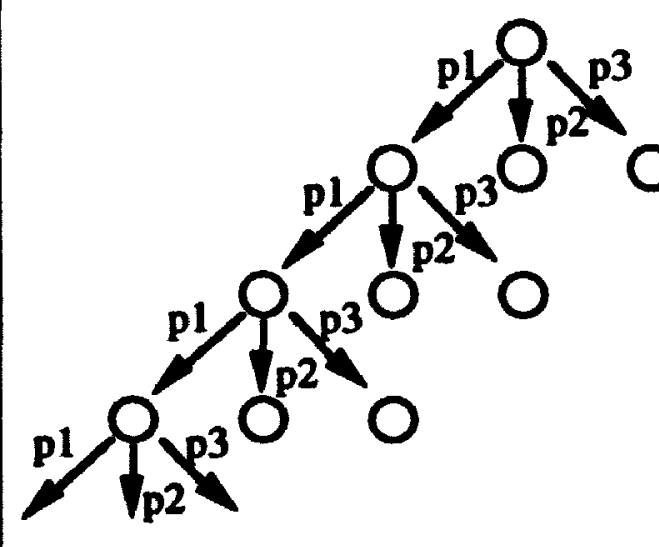

\section{Depth D}

1

Depth D-1 p2*E[ORT $\left.{ }_{D-1}\right]+$ p3*E[ORT $\left._{D-1}\right]$

Depth D-2 $\left.p 1{ }^{* p 2 * E[O R T} T_{D-2}\right]+p 1 * p 3 * E\left[O R T_{D-2}\right.$

Depth D-3

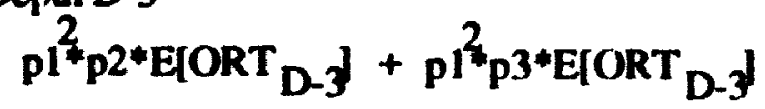

Figure 5.11 - Derviation of Recurrence Relation for Select Most Probable Path Heuristic for a k-ary Tree where arc i'th Arc Emanating From Each Node Has the Same Probability

In section 5.2.2 we noted how the optimal groupings and hence the E[ORT] for a dialog changes as the depth of the dialog graph increases. The E[ORTD] formula that we have derived above allows us to easily compare the performance of the Select Most Probable Path heuristic to the RGC algorithm as the depth of the dialog graph increases. Consider the graph in Figure 5.9. If we consider the case where we are allocating four processors we can use the $E\left[O R T_{D}\right]$ formula to compute the expected overall response time resulting from the heuristic. We find that:

$$
\begin{aligned}
& E\left[O R T_{2}\right]=1+0.6^{*} 0.1^{*} E\left[O R T_{0}\right]+0.3^{*} E\left[O R T_{1}\right]+0.1^{*} E\left[O R T_{1}\right] \\
& E\left[O R T_{1}\right]=1
\end{aligned}
$$


$E\left[O R T_{0}\right]=1$

Therefore $\left.\mathrm{E}_{[\mathrm{ORT}}\right]=1.46$

$\left(E\left[O R T_{2}\right]=1.46\right.$ from application of RGC algorithm). If we increase the depth of the tree to be four and perform our calculations again, we find that $E\left[O R T_{3}\right]=1.968\left(E\left[O R T_{3}\right]=\right.$ 1.968 from application of RGC algorithm). As we continue to increase the depth of the tree, we will observe that the change in the E[ORT] from depth $D$ to depth $D+1$ becomes smaller the further we move from the focus. Computation of E[ORT] using the formula is also useful if we have a graph with a uniform structure and homogeneous probabilities and we need to quickly decide which of the two heuristics would be better suited to the graph.

As we did in section 5.3.2, we can derive a closed formula for the expected speedup that we can achieve using $\mathbf{P}$ processors with respect to a single processor to compute a $\mathbf{k}$-ary tree dialog graph. This formula may underestimate the expected speedup since it ignores the intricacies of how processors are allocated at leaf nodes. We assume that the $\mathrm{i}^{\text {th }}$ arch emanating from each node has probability $\mathrm{p}_{i}$, and that $\mathrm{p}_{n} \geq \mathrm{p}_{\mathrm{n}+1}$. We have found this formula very useful in Chapter 7 for estimating the amount of speedup that we should expect to achieve.

The actual derivation of the speedup formula is quite straightforward: If the user must wait " $\mathrm{i}$ " times during their dialog of $\mathrm{P}$ requests for the application to respond to his/her request, the speedup relative to when 1 processor is used is P/i. The only time that a user does not have to wait for the application to compute the user request is when he/she selects the most probable node (node connected to arc with probability po). So if the user waits " $\mathrm{i}$ " times then they must have requested lix most probable node P-i times. Since the user always has to wait at least once during a dialog (at the initial focus), they must have made i-1 requests 
that did not involve the most probable node. These $i-1$ requests could involve any of the other k-1 possible requests and could happen in any order. Thus the sperdup estimation formula for the Select Most Probable Path heuristic is:

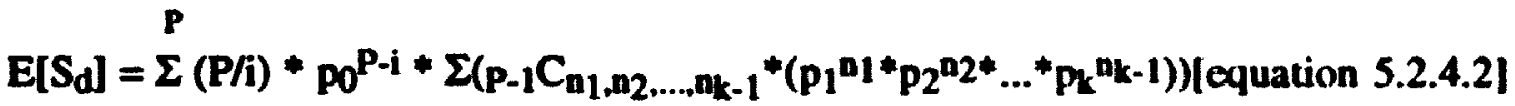

$$
\begin{aligned}
& i=1 \quad \forall n_{1} \mid n_{1}+n_{2}+\ldots+n_{2-1}=i-1 \text { and } n_{i} \geq 0 \\
& \mathbf{P} \\
& =\Sigma(P / i) P-1 C_{i-1} * P_{0}^{P-i} *\left(1-p_{0}\right)^{i-1} \\
& \text { i=1 } \\
& =\left(1 /\left(1-p_{0}\right)\right)^{*}\left(1-p_{0}{ }^{P}\right) \\
& =\left(1-\mathrm{po}_{0}^{\mathrm{P}}\right) /\left(1-\mathrm{p}_{0}\right)
\end{aligned}
$$

The Select Most Probable Path heuristic produces request groups that result in expected overall response times that sometimes are close those created by the RGC algorithm. The "all or nothing" strategy employed by the heuristic when it allocates processors is well suited to those dialogs that have a dominant path. However, when the probahilities of the nodes are approximately the same, the "all or nothing" strategy can meet with disastrous results. In situations where these is no obvious dominant path the heuristic should perform a breadth first allocation of processors. We have experimented with a heuristic that behaves in this manner. While the results obtained were superior to those achieved with the Select Most Probable Path heuristic, they did not visibly surpass the results from the Select Most Probable Nodes heuristic. Another shortcoming of the strategy employed by the Select Most Probable Path heuristic is that if a sufficiently large number of processors are available, the path length will be long. Unless a very dominant path exists, the probability that the user will not deviate from the path can be quite small. In this type of situation it would be better to only allocate processors along a single path until the probability that the user will not deviate from the path drops below some tolerance. When 
the probability drops below the tolerance alternate paths near the focus should be pursued using the remaining processors. We have experimented with a heuristic that behaves in this manner. Once again, we found that the results obtained were superior to those achieved with the Select Most Probable Path heuristic, but they did not visibly surpass the results from the Select Most Probable Nodes heuristic.

\subsection{Non-Constant Compute and Think Times}

Compute and think times do not have to specified as constant values. As we mentioned in Chapter 3, their values may be specified as discrete/continuous probability distributions or in some cases they may be unknown. In fact, dialogs where the times are unknown or inexact will be the type most often encountered. The techniques that we developed for constructing request groups can still be used when these types of dialogs are involved. In the following two sections we describe how dialogs with inexact or unknown compute and think times can be analyzed to determine request groupings. 


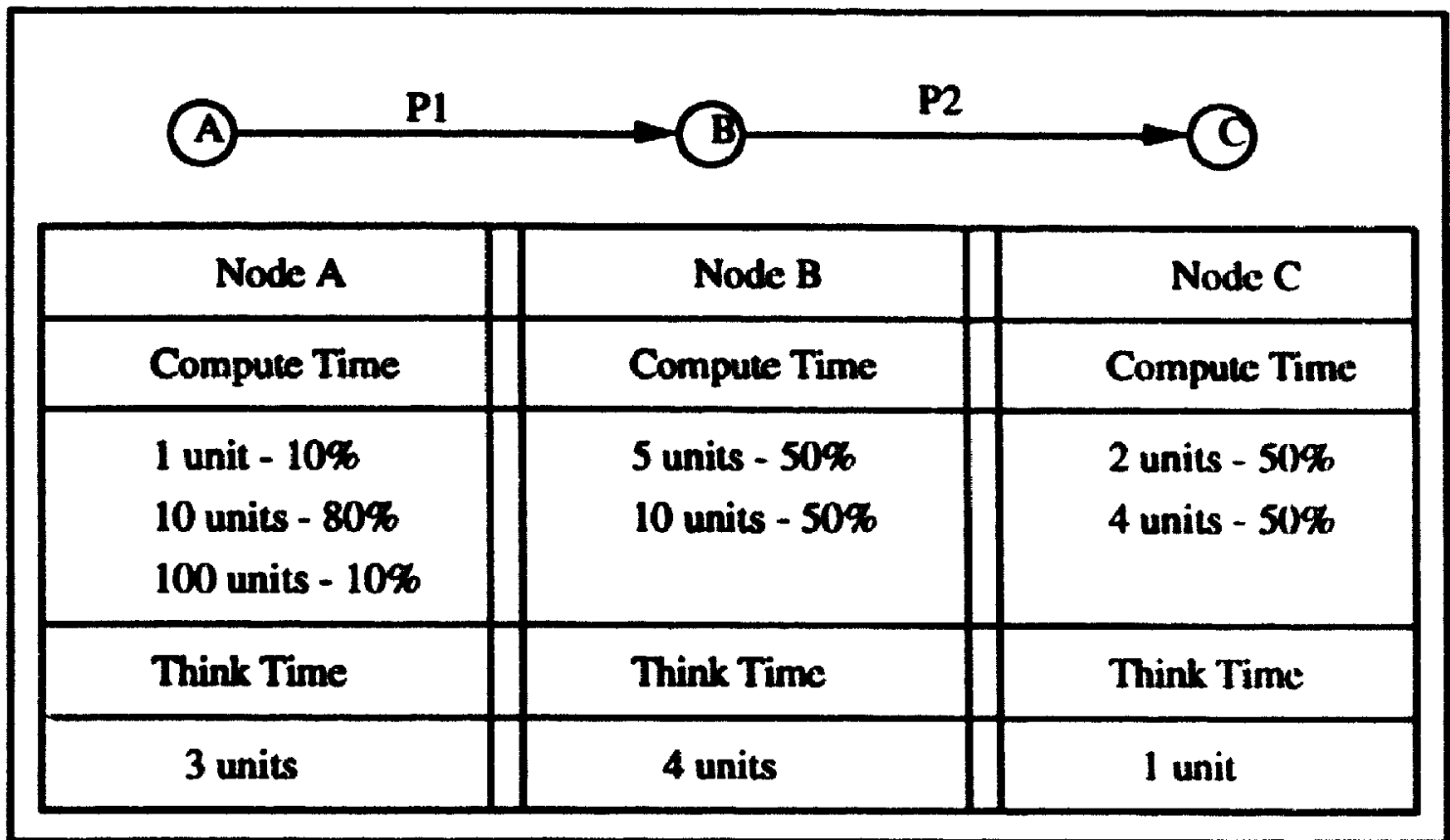

Figure 5.12 - Dialog Fragment Showing Node with Compute Times Expressed as Discrete Distributions

\subsubsection{Inexact Times}

We may often find that specifying the execution time of a request as a constant value is inappropriate. Often it will be more convenient to represent either the compute and/or think time by some probability distribution. Figure 5.12 depicts a fragment of a dialog in which the compute times are described in terms of a discrete probability distribution. The difficulty in analyzing dialogs that contain nodes whose compute and/or think times are specified by discrete probability distributions is that we cannot be certain how much time to allocate to the node since the amount of compute time required by the node varies.

As can been see from the Figure 5.12, node A will require 1 unit of compute time 10 percent of the time it is requested, 10 units 80 percent of the time it is requested and 100 
units the other 10 percent of the time. The ability to express compute and think times by probability distributions allows for the construction of more flexible dialog models that can adapt to the actions of the user.

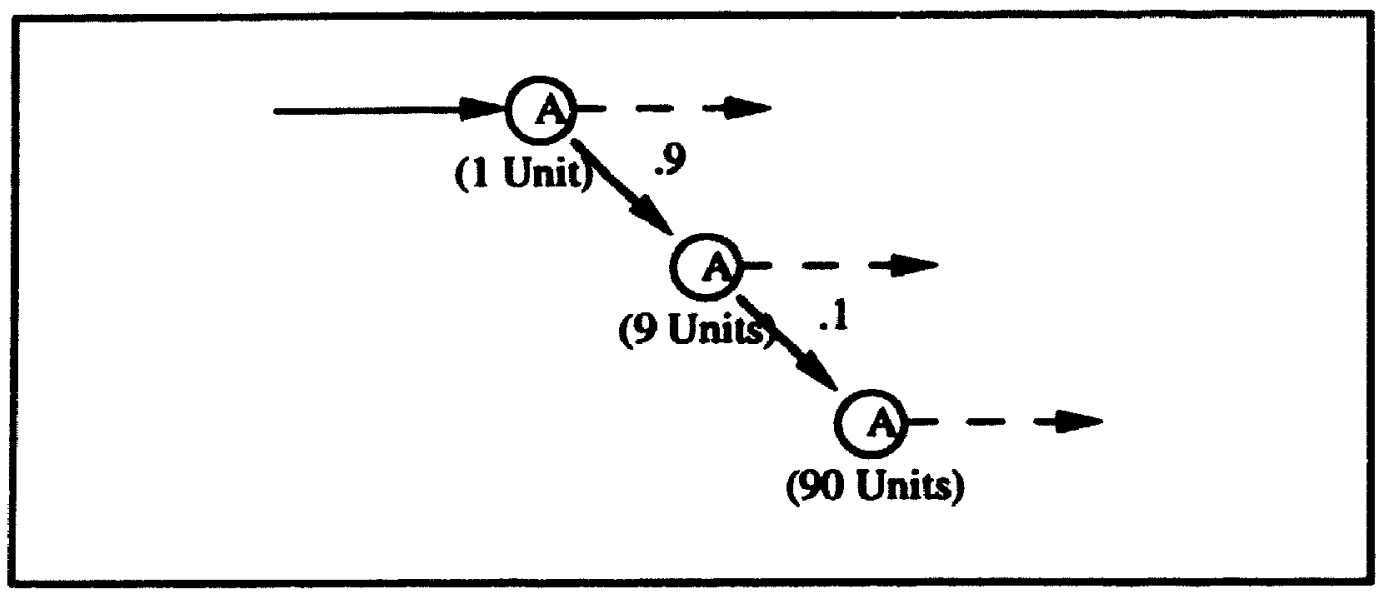

Figure 5.13 - The Expression of Variable Compute Times for Node $A$ as Listed in Figure 5.12

Variable compute and/or think times can be integrated into the model by making the following observation about what variable times actually mean. Consider the compute times listed for node $\mathbf{A}$ in Figure 5.12 again. What these times are really saying is that $\mathbf{1 0 0}$ percent of the time when node $A$ is evaluated, it will require 1 unit of compute time. Once Node A has consumed 1 unit of compute time, there is a 90 percent chance that the node will required an additional 9 units of compute time. We can see that there is a 90 percent chance that more that 1 unit is required by examining the cumulative probability function for the distribution. After the node has receive 9 additional units of compute time there is a 10 percent chance that it will require an additional 90 units of compute time. After receiving the 90 units of compute time, the node will not require any additional resources. This sequence of events can be modeled as shown in Figure 5.13. 


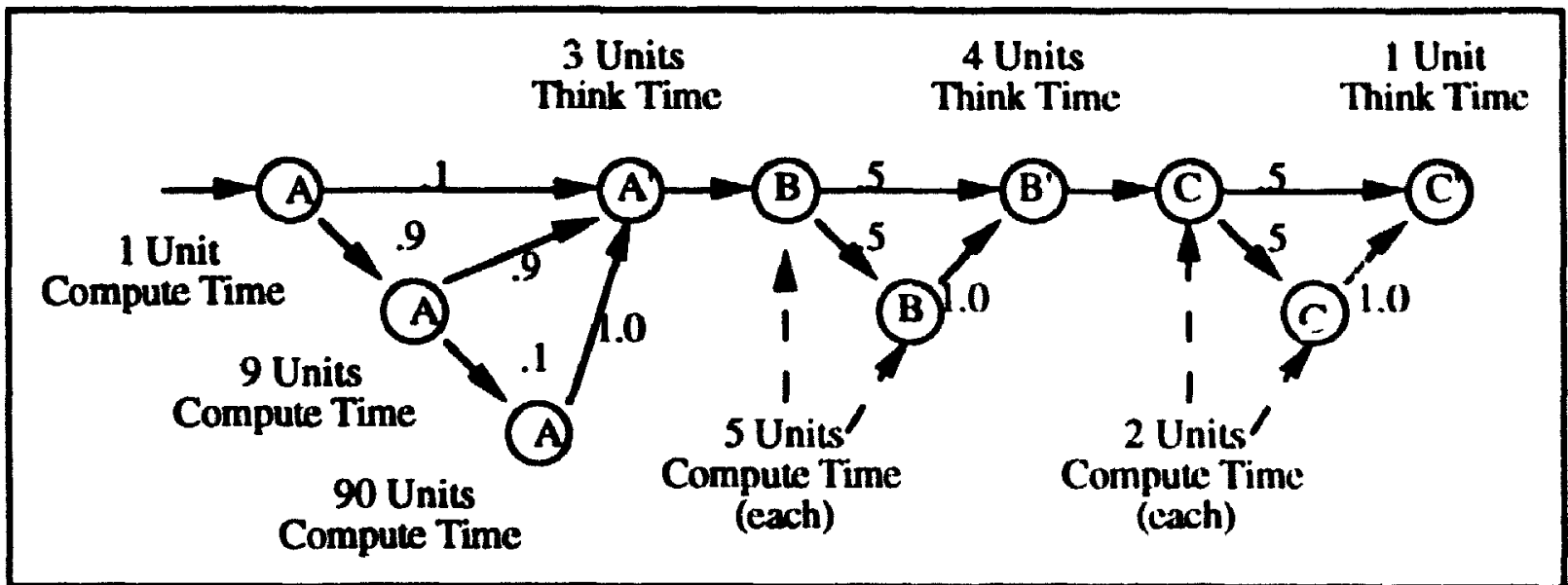

Figure 5.14- Reconstruction of Figure 5.8 To Integrate Variable Compute Times

The integration into the model is not complete since we have not addressed the issue of think times. Recall that node $A$ has an associated think time of 3 units. We cannot associate the think time with each of the A nodes is Figure 5.13. This would imply that the user would think after the evaluation of each of the nodes. Instcad we must create an additional node whose compute time is 0 and whose think time is 3 . All of the A nodes shown in Figure 5.13 will then connect to this node, to represent that the user thinks for 3 units once the result of $\mathbf{A}$ has been computed. Using this approach we can reconstruct the dialog segment shown in Figure 5.12 as the dialog shown in Figure 5.14.

If variable think times are also present then the same procedure is followed to integrate the think times into the dialog graph. Once we have constructed the dialog such that all cof the compute and/or think times for the dialog have been explicitly integrated into its structure, we can then apply the reductions introduced in Chapter 3, to remove the cycles from the dialog. 
Using reconstruction to model compute and/or think times that are not constant is only plausible to a certain degree. If the distribution is continuous then we could endeavor to model the continuous distribution as a discrete distribution, but depending on the range of the distribution or the accuracy of the approximation to the continuous distribution that we wish to achieve, the reconstruction of a node could involve the introduction of many new nodes. The unrolling of cycles introduced by reconstruction plus the unrolling of other cycles that may be present in the graph, can lead to situations where the graph size will grow exponentially. It is for this reason that we have chosen to restrict our work to dialogs where compute and/or think times are simple discrete distributions. The inclusion of cor' inuous distributions into the framework of the model is an area for future work.

\subsubsection{Unknown Times}

As in the case when compute and think times were specified by probability distributions, when we have absolutely no information regarding the expected compute and/or think times of the requests, we cannot apply the RGC algorithm to compute the optimal groupings. Fortunately, the heuristics that we have developed can still be applied to compute request groups. In the case of unknown times, we are limited in the amount of information we can produce for the groupings. We cannot compute the expected overall response time and we cannot assign request group members explicit allotments. We must schedule the evaluation of request group members solely on their relative probabilities of being requested (See Chapter 6 for discussion). 


\subsection{Summary}

In this chapter we have introduced the Request Group Construction (RGC) algorithm that computes the groupings for a user/interface dialog graph such that the overall expected response time is minimized. From our analysis of the RGC algorithm we have derived a class of heuristics that approximate the activities of the algorithm. Two heuristics from this class, Select Most Probable Nodes, Most Probable Path, have heen introduced and compared to the RGC algorithm in terms of efficiency and accuracy. We have analyred the performance of these heuristics on some restricted types of dialog graphs to determine a closed form estimate for the potential speedup that we may expect to achieve using them. We have also shown how these methods can be applied to dialogs where compute/think times may be inexact or unknown. 


\section{Chapter 6}

\section{Group Scheduling}

\subsection{Introduction}

In the previous chapter we described how to construct request groups to improve the overall response time for a user/interface dialog. In this chapter we discuss how to schedule these request groups to realize the potential response time improvement that they present. We use speculative computation to control and synchronize the execution of the request groups and their members. We identify the current deficiencies in the support for speculative computation with respect to scheduling groups and propese an extension of the sponsor model to remedy these deficiencies. A simple implementation of group scheduling using the group scheduling support presently offered by speculative computation is also presented and discussed. 


\subsection{Requirements of Group Scheduling}

The specific requirements of group scheduling differ depending on whether or not time allotments are specified for the request group members. However, one basic requirement exists regardless of the specification of time allotments; a means to start the cxecution of a task. This requirement is obvious since we do not want to start the computation of all requests in all request groups simultaneously. We want to be able to start the computation of requests when their results will be needed in the near future.

There are several other requirements that, while not being necessary, can provide for a more efficient implementation of group scheduling and productive use of processing resources. There are three such efficiency enhancing requirements:

\section{Abort Irrelevant Computation}

It is possible that some tasks in a request group may take longer to compute than the focus. Therefore, it is possible that when the user decides what request to make next, the system may still be executing eager tasks that were started by the previous request. Some of these tasks may no longer be relevant to the execution of the new request. If these irrelevant tasks are allowed to continue their execution they may prevent tasks whose computations may be relevant from executing. A means to abort tasks that are known to be irrelevant would allow us to ensure that only possibly relevant tasks are competing for resources, thereby increasing the potential that the overall response time will be improved. Scenario $A$ in Figure 6.0 illustrates such a situation. If node $\mathbf{C}$ requires longer to execute than the Focus (node A) and if the user selects node B once the focus has been computed, we 
should abort the task computing the results of node $\mathrm{C}$ and discard any of the computations it has already performed.

\section{Halt Computations}

We may not always wish to destroy all of the computations performed by a task when we stop it from executing. Instead we may wish to temporarily prevent the task from executing while still maintaining its state. We have identified the following two instances where we only want to pause the execution of a task and not discard its computations. When the user issues a new request, the request can make the computations of some tasks execuled by the previous focus irrelevant while only making other task's computations less likely. We should halt the execution of a task if the computations performed by the task are not irrelevant but are of less importance than those computations of other executing tasks so that the more important tasks can consume all of the available resources (Scenario B, Figure 6.0). We do not w ant to abort the task's computations since it is still possible that we may require its results.

Another instance where we will need to halt the execution of a task, occurs when we specify processing time allotments for request group members. The explicit assignment of processing time allotments to request group members has the advantage of making the most efficient use of processing resources. By knowing the exact processing times required by each task, we can make scheduling decisions that guarantee that the processors are always busy executing only the most probable tasks. The RGC Algorithm and the heuristics presented in Chapter 5, provide us with the most probable nodes and can guarantee that all of the request group members can receive their processing time allotments during the period that the focus node is executing and the user is thinking. The difficulty is in determining and enforcing an execution order such that each task receives exactly their allotment. This 
problem is analogous to the NP-Complete Bin-Packing problem [Garey]. In order to solve this problem, we may often have to periodically halt the execution of one or more computations so that we can interleave the execution of the tasks such that cach task receives exactly its allotment.

\section{Restart Computations}

At some point after a task has been halted we may wish to restart the task's execution. This type of situation occurs if the importance of a halted task's computations increases to a point where it is once again eligible to compete for resources. This can occur either by more important tasks completing their computations, or by the user's requests making the task more probable or other tasks less probable (Scenario C. Figure 6.0).

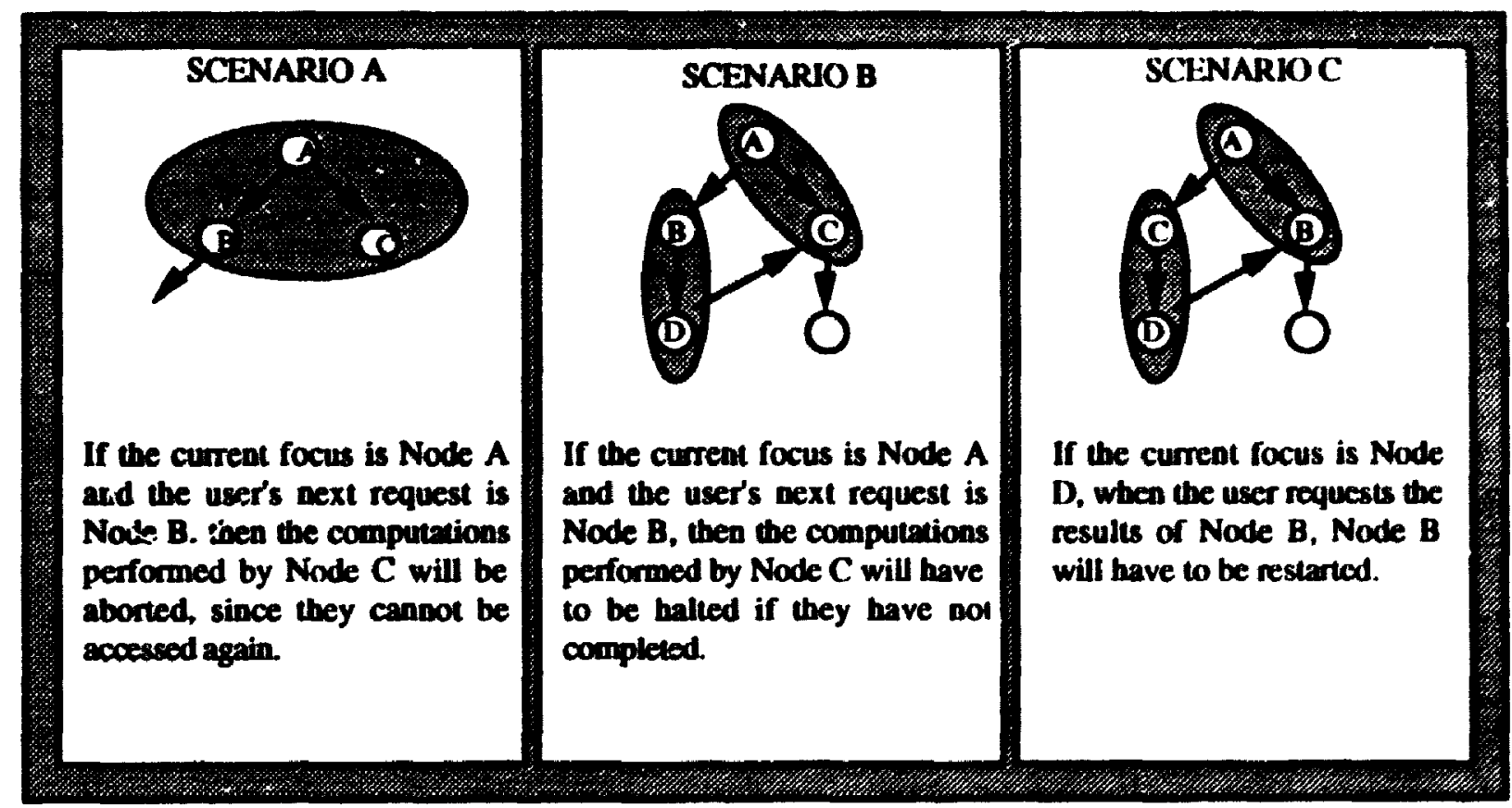

Figure 6.0 - Different Operations that may be Performed on a Node During The Scheduling Process. 


\subsection{Current Support for Group Scheduling in Speculative} Computation

Osbome's version of speculative computation provides support for the majority of the general requirements that were outlined in the previous section. The execution of a task may be halted by staying the task with the stay-group command." If the execution of a task needs to be restarted, the touch "* command will restart the execution of the task at the point where it was halted. If a task has been halted and all of the references to the task have been removed, the task is considered irrelevant and is garbage collected.

The satisfaction of the three general requirements for group scheduling is sufficient to allow the efficient scheduling of request groups composed of members whose times are unknown. However, the ability to execute tasks such that each member of a request group receives their allotment of processing time is not possible with the current support for speculative computation. In section 6.5 we propose an extension to the current model of speculative computation to support the execution of a task for a specific duration. The extended model will allow us to solve the Bin-Packing problem and therefore implement the scheduling of request groups whose members are assigned time allotments. We have not attempted to implement either the proposed extension to speculative computation. As a consequence we have not implemented a solution to the Bin-Packing problem using the extended model.

\footnotetext{
* The Multilisp constructs available for the suppon of Speculative Computation were precented in Chapter 1.

* The touch operation is one of several methods to restan the execution of a tark after it has been stayed.
} 


\subsection{Implementing Group Scheduling}

The mechanics of scheduling request groups when the member compute times are unknown is a simple process: we only need to execute enough tasks to keep all of the available processors busy. We want to be able to supply sufficient computations to occupy all of the available processors, but we do not want to flood the system with extra tasks that may not have a chance to execute. For each focus we will require some means to identify those nodes whose results should be computed in parallel with the focus. Each time a request that is being eagerly computed completes, we need to provide another task to occupy the processing resources made available by the completed request. When the result of the focus has been computed, the activities of the nodes that are being eagerly computed need to be halted so that the activities of the nodes associated with the new focus have processors upon which to execute. Figure 6.1 shows the Multilisp code used to implement group scheduling for groups with unknown compute and/or think times.

The procedure Demand-Result is responsible for determining the result of the node (the current focus) specified as its argument. The procedure creates a task to execute the members of the focus' request group and it determines the focus' result and reads the next request from the user. Once the user's next request is known, the eager execution of the focus' request group is halted and the new focus is evaluated.

The Get-Request-Group-For procedure (in line 1) returns a thunk that contains the members of the request group for the node. The Execute-Request-Group procedure is called with the request group thunk, is made into a group by the make-group function. The

\footnotetext{
* A thunk is a procedure which hos 00 arguments
} 
identifier for the group, returned by make-group function, is recorded using the group-id function so that the Execute-Request-Group procedure can be referenced. The Get-TaskFor procedure (in line 2) returns the task associated with its argument. By touching the task with the touch primitive, the Demand-Result procedure is blocked until the result of the focus' task is determined. When the result of the focus is determined, the procedure waits for the user's next request (in line 3). Once the user's next request has been determined, all of the currently executing tasks are halted (in line 4) using the function.

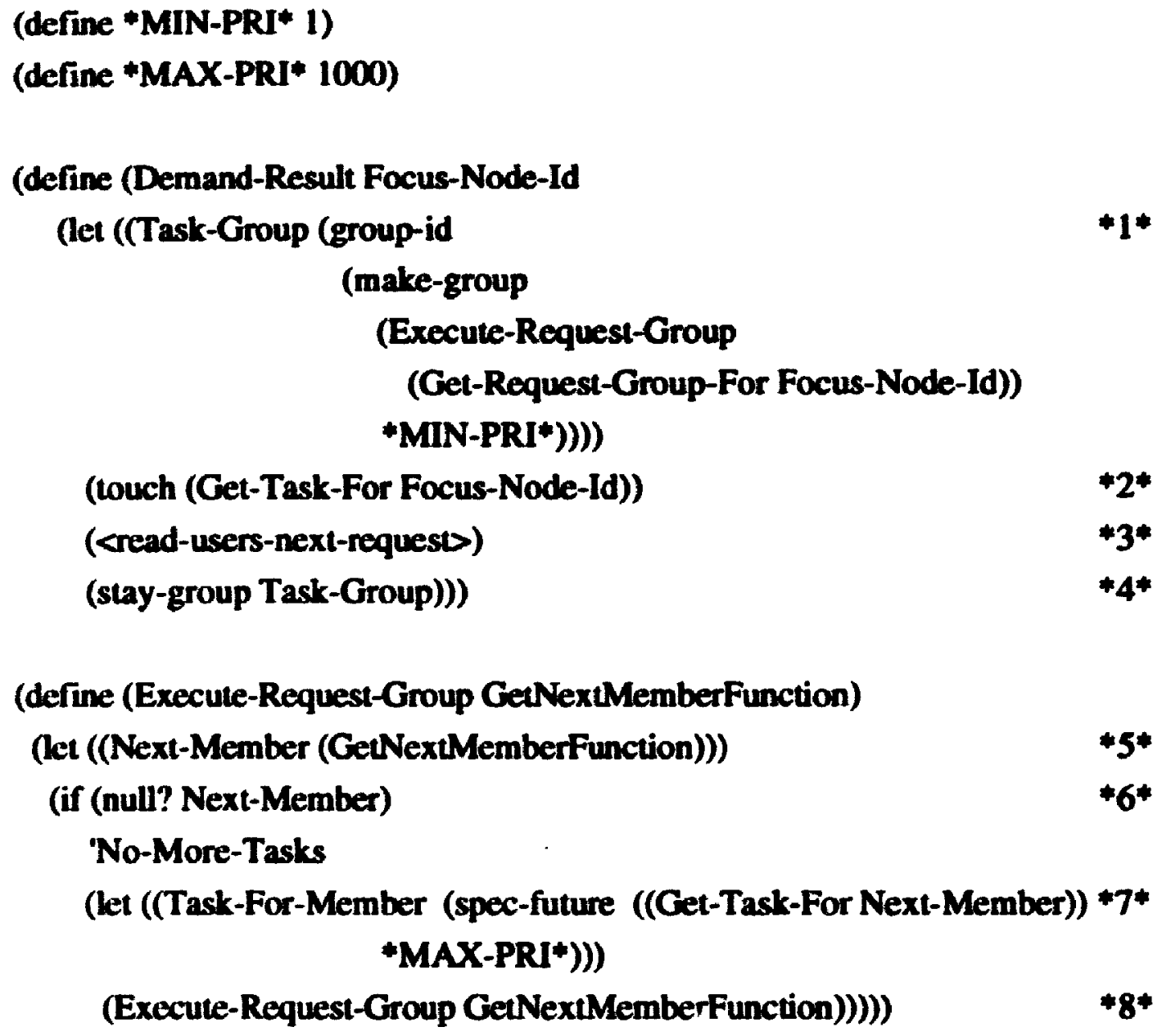

(define (Execute-Request-Group GetNextMemberFunction)

(let ((Next-Member (GetNextMemberFunction))) ) *5*

(if (null? Next-Member) $\quad * 6^{*}$

No-More-Tasks

(let ((Task-For-Member (spec-future ((Get-Task-For Next-Member)) *7* *MAX-PRI*)))

(Execute-Request-Group GetNextMemberFunction)))))

Figure 6.1 - Implementation of Group šcheduling for Unknown Compute and/or Think Times 
The purpose of the Execute-Request-Group procedure is to schedule tasks for execution when there are idle resources in the system. The procedure takes a single argument which specifies the procedure that is used to determine the identifier of the next node to be scheduled. To prevent the execution of the Execute-Request-Groups procedure from interfering with the execution of either the focus or the eager execution of its request group members, the procedure is executed at the minimum priority. This insures that the procedure will only execute when there are idle resources. The first action performed by the procedure is to determine the identifier of the next node to schedule (in line 5). If the identifier of the next node is null, this indicates that all of the nodes reachable from the focus have been totally computed (in line 6). If the identifier of the next node is valid then a task is forked using the spec-future form to evaluate the task associated with the node. The forked task is given the maximum priority and immediately begins to execuke (in Line 7). If any additional processors are available, then Execute-Request-Group will continue to create tasks to evaluate promising nodes. When all of the resources have been exhausted. the procedure will block until either it and all of the tasks it has created are stayed (in line 4) or one of the currently executing tasks completes.

The use of the priorities supported by Multilisp and the scheduling behavior of the run timc system provide us with a simple means to schedule request groups and control the execution of the member nodes when compute and/or think times are unknown. The result is an approach to request group scheduling that is both concise and elegant, and independent of the number of processors available. As we will show in Chapter 7, this simple approach is both flexible and efficient. 


\subsection{Proposed Sponsor Model Extension for Known Times}

We propose that a new type of attribute be added to Osborne's model for speculative computation to allow the sponsorship of the activities of a task or group for a fixed amount of processing time. This attribute would allow for greater flexibility in the sponsorship of tasks activities. This increased flexibility would allow us to program a solution to the BinPacking problem we described in section 6.3. This would allow us to make optimal use of resources by using task compute time information to specify time allotments when we construct request groups. Durations would also be potentially useful when speculative computation is used for implementing "competitive parallelism" [Suttner]. With competitive parallelism we execute several different approsches to solving a problem in parallel, hoping that one of the approaches will quickly arrive at a solution. If we know that one of the approaches tends to either arrive at a result in a specific time or never arrive at a solution, it would useful to be able to sponsor the activities of this approach only until it arrived at a result or it excoeded its time limit. By aborting the execution of the approach once it has exceeded its time limit, we would make the resources held by the approach free for the other approaches to consume.

We considered several implementation independent issues when we undertook the design of how duration should be added to the current model of speculative computation. The issues included:

1. Since the activities of a task are being sponsored for a duration, how should the duration be measured? The amount of processing time consumed by the task would most probably not be the same as the elapsed "wall clock" time during the 
same period. This type of phenomenon would be particularly evident in a system where each task does not have a dedicated processor.

2. Since the task is being sponsored for a fixed duration, how can the sponsor determine if the task has arrived at a result or if it has run out of processing time? Since the sponsor is not demanding the result of the computations performed by the task, the sponsor cannot tell if the task has actually arrived at a result. It may be important for the sponsor to know if a task has arrived at a result if the sponsor has some extra resources and wants to use the resources to sponsor the activities of a task that is not yet complete. Knowing that a task had already arrived at a result would immediately allow the sponsor to eliminate the task from consideration.

3. Since the sponsor is not actually demanding the result of a task, should the execution of the sponsor be blocked while it sponsors the execution of the task or should the sponsor execute in parallel with the activities of the sponsoned task? As we have already mentioned, the touch primitive is the primary means of sponsoring the activities of a task. When a sponsor touches a task, the sponsor is blocked from executing until the sponsored task arrives at a result. The act of blocking the sponsor provides a means of synchronization between the sponsor and the sponsoree. Another means of sponsoring the activities of a task is through the use of classes [Osbome]. Classes provide a means of sponsorship without requiring the sponsor to block. 


\subsubsection{Adding Duration Attribute}

Engines were implemented in the Scheme programming language by Haynes and Friedman [Haynes]. Engines support the timed preemption of computations. There are two stages to using an engine to control the duration of computations: the engine must be built and then the engine must be fueled. When an engine is created, it is given the computation that is it to perform when it is running. The constructed engine requires three arguments to be invoked: the maximum amount of time to work on a computation, a procedure to invoke should the computation finish (i.e., a continuation) and a procedure to invoke if the engine does not finish its computations before the maximum time is exhausted (i.e., a continuation).

We propose a similar notion be adjed to the current model of speculative computation. These engines retain much of the same flavor they have in sequential Scheme, but they have been integrated with the current suppont for speculative computation and the notion of sponsorship.

In a parallel environment thene are several issues that did not need to be taken into account in a sequential environment. First, it may be desirable to allow several sponsors to sponsor the computations that are being performed by a task associated with an engine. Within the sequential scheme environment, this type of situation was not possible. We introduce the notion of ergine objects to facilitate the sponsorship of a task encapsulated within the engine by several sponsors simultaneously. Second, in the sequential environment the execution of an engine could only be preempted when the engine consumed all of its fuel. In a parallel environment, an engine can be preempted not only from running out of fuel but 
by other tasks running at higher priorities. Because of this, it may take varying amounts of wall clock time for an engine to consume a specific amount of fucl. Lastly. Haynes' and Friedman's engines consumed fuel and either returned the result of their computation and any remaining fuel or they stopped without arriving at a result. In a parallel environment where there may be multiple tasks simultaneously sponsoring the activities of an engine, returning unused fuel is not a trivial operation.

We refer to the fuel that a sponsor supplies to an engine as coming from a sponsor's storage-tank. Any task may create one or more storage tanks containing a specific amount of fuel (see below). A sponsor allocates fiel to an engine by providing the engine with access to a storage-tank and a specific total amount of fuel that can he drawn from the storage-tank. In the event that an engine computes a result, the engine returns all of the fuel it has not consumed to the storage tanks of th various sponsors that have sponsorred its computations. In order to allow an engine to keep track of the various sponsors that have allocated the engine fuel, we also introduce the notion of fuel reservoirs. Fucl reservoirs are different from storage tanks in that they do not explicity ccatain fucl but instead kexp a list of fuel locations and how much fuel can be taken from each location. For each engine we keep track of the amount of fuel supplied by a sponsor and the location of the storage tank that contains fuel. Keeping fuel sources separate in a parallel environment is important for two reasons: the identification of sponsors to return unspent fucl to and what order fucl from sponsors of different or equal priorities should be consumed.

We propose that the following three new constructs be added to the Multilisp language for the support of duration sponsorship: 
(BUILD_ENGINE sponsoree tank-size continuation) : BUILD_ENGINE creates an engine object for the evaluation of the task/group thunk specified as the sponsoree. The engine has an associated fuel tank that will have the capacity specified as tank-size. When an engine is constructed, its tank is empty. The continuation specifies what the engine should do in the event that it runs totally out of fucl or the task which is being sponsored arrives at a result. As opposed to Haynes' and Friedman's engines, our engines only use a single continuation. We feel that the use of two continuations is not necessary and that the specification of a single continuation to interpret the validity of results is sufficient. The continuation takes two arguments: status and result. The status argument indicates whether the sponsored task has completed its execution. The result argument contains the result of the sponsored task (if any). If the status argument indicates that the task has not completed its computations, the value of the result argument is undefined. The continuation can be used to interpret and return the result, actively seek other sponsors for the engine, or to refuel the engine. The continuation executes at the same priority as the sponsor from whose storage tank the engine's current supply of fuel has been drawn. It is possible that an engine may conceptually fuel itself. This feat is possible since the task sponsored by the engine may acquire fuel from some other tasks and place the fuel in the tank of its own engine by using the fuel-engine construct (see helow). Any task may create an engine using the build-engine construct.

(FUEL_ENGINE engine storage-tank fuel-limit): FUEL_ENGINE provides the engine specified by engine with fuel. the amount of which is specified by fuellimis. The fuel is drawn from the storage tank specified by storage-tank.. If the 
fuel limit specified is greater than the tank capacity of the engine, the engine will (if required) fill its tank on multiple (ccasiions until it has consumed all of the fuel allocated. If the sponsor allocates more fuel than the sponsor has in storage, the engine will draw as much fuel as possible and move the sponsors fuel promise to the end of the engine's fuel resevoir list in hopes that at some later time, the sponsor will make goad on its promise.

(BUILD_STORAGE_TANK fuel-in-storage): BUILD_STORAGE_TANK creates : fuel tank that contains fuel-in-storage units of fucl. Any task or enginc can create a storage-tank object and allow access to it by other tasks or engines. We have no fixed source of fuel. We do not limit the size or number of storage tanks that a task or engine can allocate. When the tank is created, the fuel is drawn from a global storage tank of infinite capacity that is created when the root task for the system is created. We do not limit the amount of fuel that may be placed in a storage tank when it is created. Currently, the notion of fucl is used primarily by the sponsor to meter the amount of resources it alloxates to other tasks. In the future after experimenting with the model, we may wish to impose some restrictions on how much fuel a task may withdraw from the global storage tank.

Figure 6.2 summarizes the additions made to the sponsor mode for the support of the duration attribute. When the engine receives fucl, it fills its tank or lakes all of the fuci given to it (which ever is less), records a pointer to the storage-tank from which the fucl was drawn and begins the evaluation of the task that was specified when it was created. If the engine arrives at a result, it returns all of the fuel that remains in its tank to the storagetank that it was drawn from. When the tank empties, the engine will try to refill its tank 
from storage-tank of the highest priority task offering it fuel. If no tasks provide fuel for the engine, the engine will invoke the continuation that it was given when it was built. The engine will provide the continuation with a status of "incomplete" and an undefined result.

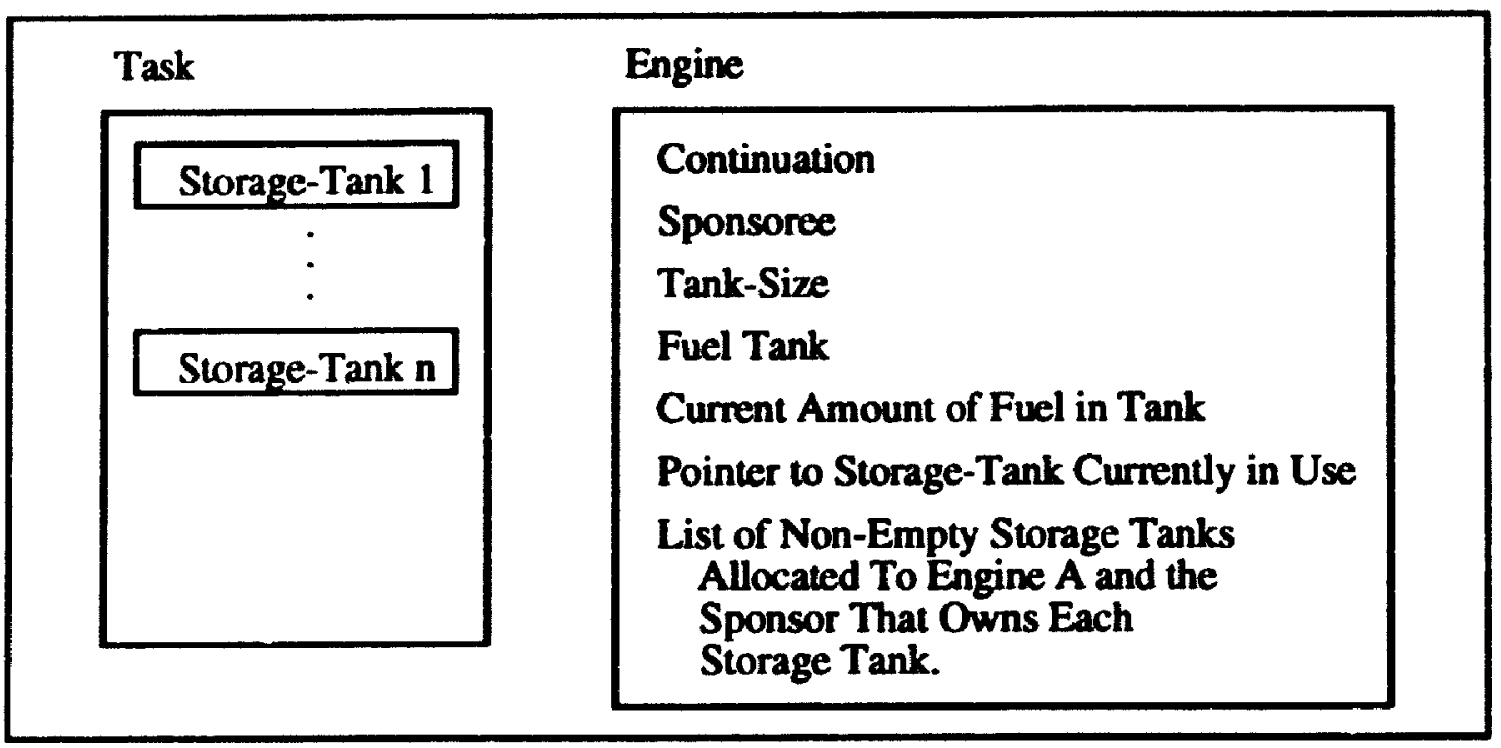

Figure 6.2 - Task/Engine Components for Support of Duration Attribute

In the cvent that the task being evaluated by the engine computes a result, the engine returns the fucl in its tank to the last storage-tank accessed and invokes its continuation with a status of "complete" and a result equal to the result of the task. Any future attempts to sponsor the activities of the engine will be "ignored". The engine will "ignore" the sponsorship attempt, by calling the continuation with the result of the task and a status of "already complete". The status "already complete" is used in place of "complete" to allow continuation to know that it has already been invoked at least once, with a valid result.

Our model addresses the fundamental implementation independent issues as follows: 
1. We have chosen the duration for processing time to be measured in processing time not real time. Processing time is more applicable since we cannot guarantee that the task will execute as soon as it is sponsored. We may also use processing time as a pseudo real time measurement if we have a machine that has idle processing resources.

2. We can determine the result of the task by either having the continuation inform us or by touching the task. There is no real need to know if the fucl that we ane giving the engine is enough to compute the result of its task. If we allocate fuel to an engine that has already computed a result, the fuel is not lost, it is simply placed back in the storage-tank. If we are really concemed about the result of the task then we should touch sponsor the task to determine its result.

3. The action of sponsoring $\rightarrow$ task/group for a specific duration is asynchronous. Since the sponsor is not concerned about the result of the task, there is no reason to wait for the engine to use its allocation. In addition the sponsor cannot guarantee how long it would take the engine to consume all of its fuel so the sponsor could be suspended for quite some time. If the sponsor really wants to be synchronized with the task, it should make the rest of its activities the continuation that is supplied to the engine when it is constructed. This would result in the sponsor being restarted once the engine has run out of fuel or the task has computed a result.

\subsubsection{Group Scheduling Using Duration Attribute}

We have chosen not to implement the duration attribute. We have made tisis decision for two reasons: Implementing the attribute and properly incorporating it into the existing 
implementation of the speculative computation model would be time consuming. Actually implementing the engine construct and experimenting with a Bin-Packing algorithm would not add significant insight into the general question of how to best reduce response time using speculative computation. The results that would be obtained would most likely not be significantly better than those obtained when Bin-Packing is not used to maximize processors utilization. The use of duration for scheduling request group members is only useful when the members of the request group are assigned specific duration. Since we have not implemented the engine construct, the following implementation should only be considered as a proposal.

It is not significantly more difficult to implement request group scheduling when time allotments are specified. The scheduling only requires a few simple modifications of the implementation used when members did not have specified allotments. By employing a Bin-Packing algorithm to determine the order to execute tasks and their duration, we can schedule request groups whose members have specific time allotments such that each member receives its assigned allotment. Instead of simply scheduling the next request to eagerly compute until it either computes a result or all of the actively eager computing requests are stayed, we use an engine to control the duration of the task.

In our implementation, each node contains an engine that encapsulates its task. By having the Execute-Request-Group procedure fuel the engine, instead of directly sponsoring the task, we provide the necessary means to schedule the results of the bin-packing algorithm. We no longer need to halt the activities of the Execute-Request-Group procedure since the activities of the procedure terminate when they have scheduled the request group for the focus. Figure 6.3 shows the Multilisp code that could be used to implement group scheduling for nodes with specified time allotments. 
(define *MIN-PRI* 1)

(define *MAX-PRI* 1000)

(define Storage-Tank (BUILD_STORAGE_TANK <maximum-integer $>$ ))

(define (Demand-Result Focus-Node-Id

(let ((Task-Group (spec-future

(Execute-Request-Group

(Apply-Bin-Packing-To-Group

(Get-Request-Group-For Focus-Node-ld)) * *1* *MIN-PRI*))))

(touch (Get-Task-For Focus-Node-Id))

(<read-users-next-request $>)$ ))

(define (Execute-Request-Group GetNexiMemberFunction)

(let ((Next-Member (GetNextMemberFunction)))

(if (null? Next-Member)

'No-More-Tasks

(let ((Allotment (Get-Allotment-For Next-Member))) (fuel-engine

(Get-Engine-For (Get-Node-Id Next-Member))

Allotment

Storage-Tank))

(Execute-Request-Group GetNex(MemberFunction)))))

Figure 6.3 - Implementation of Group Scheduling for Known Compute and/or Think Times

The Get-Request-Group-For procedure (in line 1) ret'rns the request group for $U_{\mathrm{h}}$ c curr:nt focus. A Bin-Packing algorithm is applied to the request group to determine the order in which to schedule the members of the request group so that the members receive their time allotments. The output from the Bin-Packing algorithm is given to the Execute-Request- 
Group procedure. The Get-Allotment-For procedure (in line 2) returns the fuel allotment for the next task to be scheduled. The next task in the request group is executed by placing the amount of fuel retumed by the Allotment-For procedure in the task's engine (in line 3).

\subsection{Summary}

The current sponsorship model of speculative computation is sufficient to allow the implementation of request group scheduling where the members of the request groups are not assigned processing time allotments. When request groups members are assigned processing time allotments, the current sponsorship model is deficient in that it is not possible to sponsor the activities of a task/group for a fixed duration. We have proposed a duration attribute as a means to sponsor the activities of tasks and groups for fixed duration. 


\section{Chapter 7}

\section{Application and Results}

In this chapter we consider an application which uses speculative programming wechniques and the request group heuristics we have developed, to reduce the overall expected response time. This application illustrates the use of speculative computation and its potential for reducing the overall response time. All of the exccution times listed in this chapter we obtained running the Multilisp+SC language interpreter on a DECStation $5000 / 200$.

\subsection{The Application}

In Chapter 1 we introduced the Dungeon Explorer game as one of our two example applications. Play in the Dungeon Explorer game consists of the user exploring roxums in a dungeon. During his/her exploration the user has chance encounters with creatures and finds treasure. The version of the game that we have implemented for our experiments is a 
simplified version of the general game introduced in Chapter 1. In the version implemented in this Chapter, the user explores a maze in search of a way out. The goal of the simplified game is to find an exit from the maze, rather than meeting creatures and accumulating wealth.

After the user has entered the parameters that specify the structure of the maze (see section 7.2 for a description of the parameters), play starts by the user being placed in the center of the maze. The user is shown the names of the doors that he/she may use to exit the current room. The doors are "one way", so that once the user has chosen an exit route from the ;om, he/she may not revisit the room to try another exit. When a room is selected, the user is immediately placed in the next room. In the event that the room has not already heen pre-computed at the time of the user's arrival, the user is delayed from selecting an exit from the room until the room is constructed. When the user selects a door that is an exit, the game is finished and the total time that user spent waiting for rooms to be constructed is displayed.

\subsection{Implementation}

The implementation of the Dungeon Explorer game consists of two distinct parts. The first part is concerned with defining the characteristics of the maze and the construction of the mare. The second part is concerned with the actual play of the game. We will discuss both of these parts in detail.

When the game is first started by the user, the user is asked for information that is to be used to define the characteristics of the maze that he/she will try to escape from. The information the user is request to supply includes: 


\section{Number of Processors}

This is the number of processors that the application should assume are available to compute rooms. The number of processors is required by the Multilisp+SC simulator so that it knows how many processors should be simulated. The user is allowed to enter any natural number for the number of processors.

\section{Rooms In The Maxe}

This is the total number of rooms that the maze should contain. The user is allowed to enter any natural number for the number of rooms in the maxt.

\section{Number of Doors per Room}

This is the number of doors that the user will have to chose from when he/she decides which room to visit from the current room. All rooms, except exit rooms (these have no doors) will have the number of doors specified. The number of doors per room can be any natural number.

\section{Compute Time Distribution}

This defines the time distribution for the compute time that a room can require to be constructed. A single unit of compute time is equal to the amount of time that it takes to perform a call to a procedure that subtracts one from its integer argument and returns the result. The minimum and maximum values entcred either define the limits of an integer uniform distribution or define a two-valued discrete distribution that are used to determine the compute time for each room as it is added to the maze. The values entered by the user must be positive irteger 
values and the value of minimum compute time must be less than or equal to the value of the maximum compute time.

When we construct the maze, we assign random probabilities to the doors in each room to represent the probability that the door is opened by the user. These are the probabilities used during the analysis of the maze to determine the membership of request groups. As we will discuss is section 7.3 , we have restricted the randomness of the probability assignments in some of our experiments.

We assume that the think time required by the user is zero. We have made this assumption bocause many games do not allow the user to think between moves; either the user immediately docides what action to take next or the game continues to progress without the user's input. From the perspective of performing eager computation, the assumption of zero think time is a worst case scenario. With zero think times, we can only eagerly construct rooms while the user is waiting for a room that he/she has entered to be constructed. If think times were non-zero, we could exploit the time periods when the user is thinking by using all the available processors to eagerly construct rooms.

Even though we know at the time we analyze the maze to construct request groups what the compute time assigned to each room is, we do not use this information to aid in the construction. We have elected to assume for the purpose of analysis that the compute time for cach room is unknown. We have made this assumption since the knowledge of the compute times would be of no benefit since we have not implemented the solution to the bin-packing problem (discussed in Chapter 6). 
(define (Dungeon-Explorer)

(display "How Many Processors?")

; read number of processors

(set! *SYSTEM-PROCESSORS* (read))

; dedicated to game.

(if (or (not (number? *SYSTEM-PROCESSORS*))

(<1 *SYSTEM-PROCESSORS*))

(error "Invalid System Processors"))

(display "How Many Rooms?")

; read number of rooms in mare

(set! TOTAL-NODES-MAXIMUM (read))

(if (or (not (number? *SYSTEM-PROCESSORS*))

(< 1 TOTAL-NODES-MAXIMUM))

(error "Invalid Number of Rooms"))

(display "How Many Doors per Room")

; read number of doors/rowm

(set! DESCENDANTS (read))

(if (or (not (number? *SYSTEM-PROCESSORS*))

(< 1 DESCENDANTS))

(error "Invalid Number of Doors"))

(display "Discrete or Continuous Distribution (D)?")

(let ((Type (read)))

(if (or (eq? Type 'D) (eq? Type 'd))

(begin

(display "What is the Two Compute Times?")

(set! FIRST-EXEC-TIME (read))

; first value

(set! SECOND-EXEC-TIME (read))

; second value

(display "What are Their Respective Probabilities?")

(set! FIRST-PROB (read))

(set! SECOND-PROB (read))

; first value

(if (or (not (number? MIN-EXEC-TIME))

(not (number? MAX-EXEC-TIME))

(not (number? FIRST-PROB))

(not (number? SECOND-PROB))

$($ not $(=1.0(+$ FIRST-PROB SECOND-PROB $))))$ 


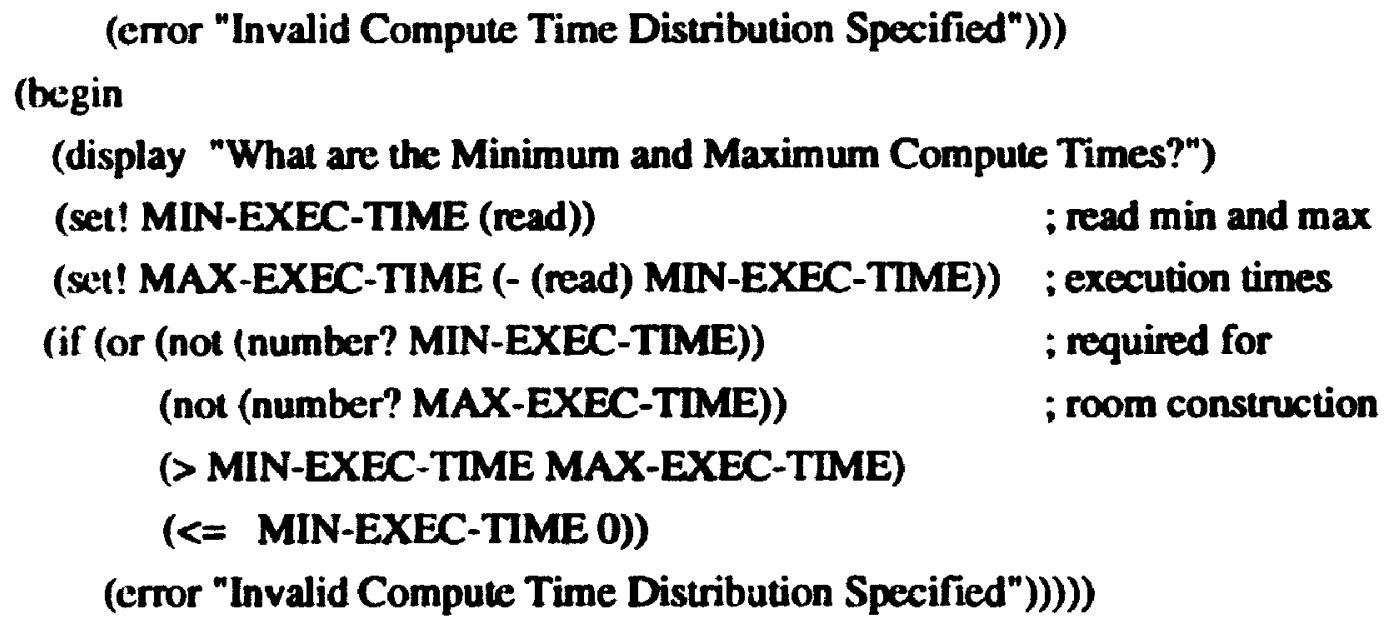

(display "Constructing The Maze")

(Construct-Maze)

(Enter-Room INITIAL-ROOM))

$$
\begin{aligned}
& \text {; build the maze } \\
& \text {; pace the user in room } 0
\end{aligned}
$$

Figure 7.0 - Multilisp+SC Code for Reading Maze Parameters

Figure 7.0 lists the Multilisp+SC code used to implement the initialization section of the Dungeon Explorer game. If any of the values entered by the user are invalid, (for example a negative number of processors), the game is aborted and the user must start over. Once the user has successfully answered all of the prompts, a maze is constructed and the user is placed in room 0 where the actual exploration begins.

There are five basic steps that the application continues to iterate through until the user loxates an exit:

1. Stan the eager construction of rooms that comprise the request group of the room that the user is currently located.

2. Finish the construction of the room that the user is currently in.

3. Display the adjoining rooms that the user can visit next. 
4. Read the name of the room that the user would like to enter next.

5. If the room is an exit, end the game.

The actual portion of the application that is responsible for contro!ling the eager computation of rooms and the coordinating the user's movements through the maze is very similar to the code that was introduced in Chapter 6 for scheduling request groups. The major difference is the use of room objects to encapsulate all information specific to a room. This information includes:

- adjacent rooms

- compute time for room's construction

- the task associated with computing the view of the room

- the request groups associated with the node

\begin{tabular}{|l|l|}
\hline Message & Value Retumed \\
\hline GetNextMemberFunction & $\begin{array}{l}\text { A parameterless procedure that returns the name of } \\
\text { the next room in the request group to be executed }\end{array}$ \\
Request Task & $\begin{array}{l}\text { A procedure that is responsible for the } \\
\text { construction of the room. } \\
\text { AdjacentRooms }\end{array}$ \\
$\begin{array}{l}\text { Alist of the names of all of the rooms reachahle } \\
\text { from the current room }\end{array}$ \\
\hline
\end{tabular}

Figure 7.1 - Messages Accepted by Room Objects and Their Return Values

Figure 7.1 lists the various messages accepted by a node object and the values that are returned. As we can see from this Figure, the actual task that constructs the room is encapsulated within a future object. This allows us to halt and resume the execution of the task while letting the run-time system maintain the current state of the task's computations. When the room object is created, the task that constructs a room is encapsulated within a 
future and has its priority set to zero so that it does not actually start to execute immediauely. When the RequestTask message is sent to a room object, it returns a pointer to the future object that encapsulates the room's construction task. Figure 7.2 lists the Multilisp+SC code that schedules the cager construction of rooms and controls the movement of the user.

When a user enters a new room, the first operation is to decide if the user has found an exit (the Exit? function in line 1). If an exit is found then the game ends. Otherwise the program retrieves the object for the current room (in line 2 ). The code in line 3 is responsible for creating a task to schedule the rooms that are in the current room's request group. The Execute-Request-Group procedure is supplied with the thunk that returns the next member in the ronm's request group each time it is invoked. We use the make-group procedure to encapsulate the Execute-Request-Group procedure so that the program can halt the execution of the procedure (stay-group in line 7) when the user wants to enter a new room. Once the program has started to eagerly construct the rooms in the request group, the program finishes the construction of the current room (in line 4). It is the time that we spend executing this particular line of the program that we are trying to minimize. Once the room has been fully constructed the applications asks the user what adjoining room he/she wants to visit next (in line 5). The application reads the name of the next room from the user (in line 6), halts the Execute-Request-Group procedure that was started at line 3. 


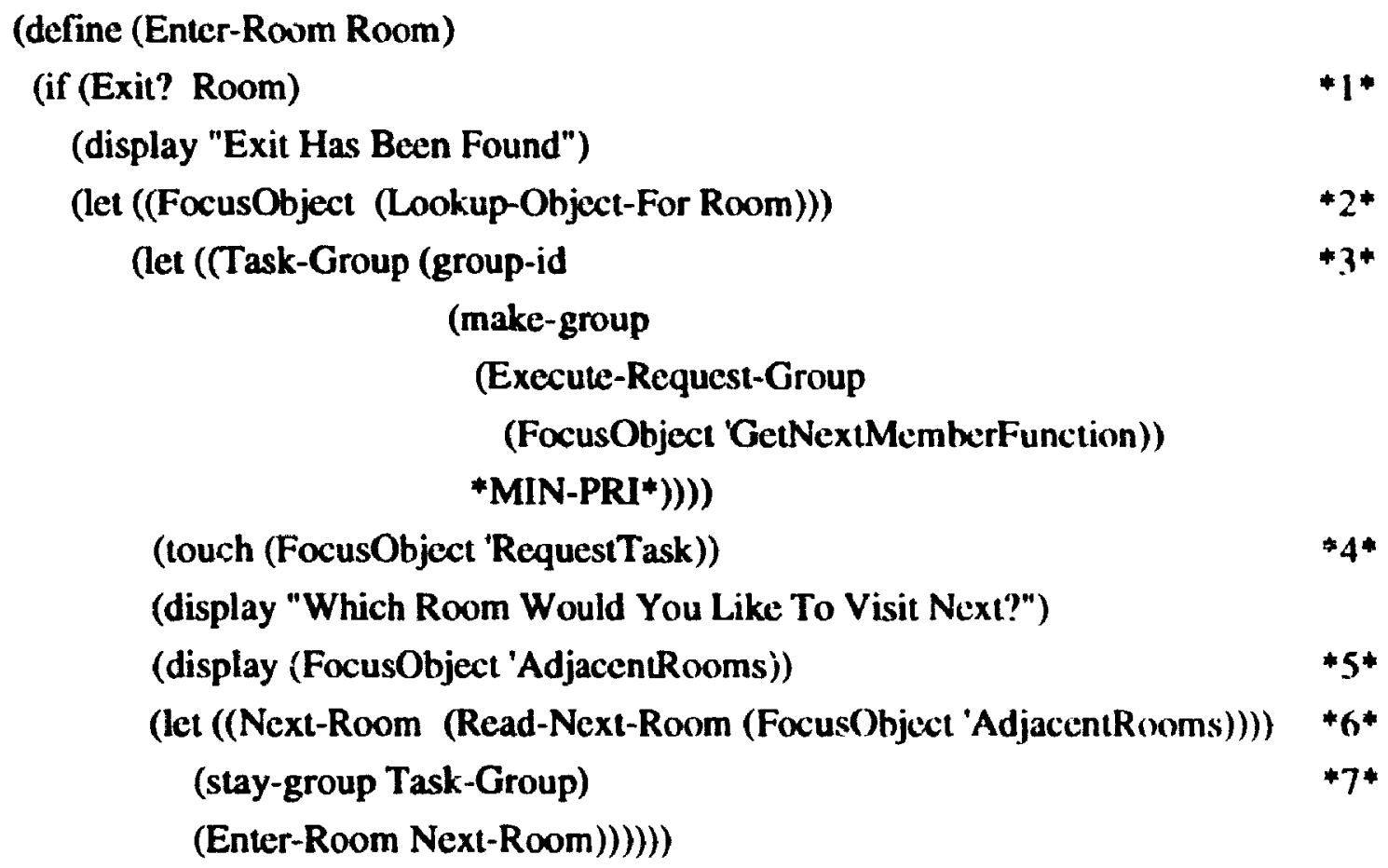

Figure 7.2 - Multilisp+SC Code for Dungeon Exploration Game

\subsection{Experiments}

We have conducted several experiments involving the Dungeon Exploration Game. The goal of these experiments was to determine the merit of using speculative computation $t$, improve the overall response time for the game. We have collected results for speculative (halt the eager construction of rooms that are found to be irrelevant), mandatury (do not halt 
the eager construction of a room once it has started), and sequential (do not perform any cager construction of rooms) versions of the game.

Our experiments centered around varying three parameters input to the game:

\section{Number of doors per room}

2. Compute Time Distribution Parameters.

3. Number of Processors used by the Multilisp+SC simulator.

In all of our experiments we used three basic maze sinctures with 2 doors per room, 3 doors per room, and 4 doors per room respectively. Each of these structures contains exactly $\mathrm{N}^{3 \mathrm{P}-1}$ rooms (whert $\mathrm{N}$ is the number of doors/room and $\mathrm{P}$ is the number of processors used). We chose this formula for determining the number of rooms because it provides an adequate number of rooms to require the user to explore several rooms before he/she finds an exit. We also varied the number of processors simulated by the Multilisp+SC simulator for values of 2,4 , and 8 .

We used three different types of probability distributions in conjunction with the $2,3,{ }^{\prime}$ door/room mazes. We refer to the first type of distribution as the Equal Visit Probabilities distribution. In this type of distribution, each door in the maze has the same absolute probability. For example, in the 3 door/room maze the probability of each door is $1 / 3$. We refer to the second type of distribution as the Dominant Path Present distribution. In this type of distribution. one of the doors leading from a room has a 0.90 probability of being selected, while the remaining 0.10 of the time one of the other doors is selected. For example, in the 3 door/room maze the probabilities assigned to the doors would be $90 \%$, 0.05, and 0.05. We refer to the final type of distribution as the Random distribution. In this type of distribution, each of the doors leading from a room is assigned a probability 
that is a multiple of 0.10 , and the sum of the probabilities for all doors leading from the room is 1.0 . The minimum probability that a door may he assigned is 0.10 .

For each experiment, we conducted 10 random walks through each of the possible "doorsper-room/simulated-processor" combinations. We report the average execution time for set of 10 walks and the associated $90 \%$ confidence interval, and the resulting speedup relative to the sequential version. We performed each experiment twice, once using the Select Most Probable Nodes heuristic to construct the request groups and otce using the Select Most Probable Path heuristic. We also condurted two experiments that compare the specedup achieved using the Select Most Probable Nodes heuristic to those generated hy the RGC algorithm.

Expected Speedup

\begin{tabular}{|c|c|c|c|c|}
\cline { 2 - 5 } Doors & Processors & $\begin{array}{c}\text { All Paths Equal } \\
\text { Select Most } \\
\text { Probable Nodes }\end{array}$ & $\begin{array}{c}\text { All Paths Equal } \\
\text { Select Most } \\
\text { Probable Pauh }\end{array}$ & $\begin{array}{c}\text { Dominant Path } \\
\text { Sclect Most } \\
\text { Probahle Path }\end{array}$ \\
\hline 2 & 2 & 1.5000 & 1.5000 & $1.9(\times)$ \\
& 4 & 2.2500 & 1.8750 & 3.4390 \\
& 8 & 3.1250 & 1.9920 & 5.6954 \\
\hline 3 & 2 & 1.3333 & 1.3333 & $1.9(\times)$ \\
& 4 & 2.0000 & 1.4815 & 3.4390 \\
& 8 & 2.4440 & $1.5(\times) 0$ & 5.6954 \\
\hline 4 & 2 & 1.2500 & 1.2500 & $1.9(\times)(0)$ \\
& 4 & 1.7500 & 1.3281 & 3.4390 \\
& 8 & 2.1875 & 1.3333 & 5.6954 \\
\hline
\end{tabular}

Table 7.0 - Expected Speedups for Heuristics on a Dialog with all Nodes having same Compute Times

\subsection{Results}

In this section we present the results of our experiments. Table 7.0) lists the specdups expected by both the speculative and mandatory versions of the game if all rooms have the 
same compute time and all doors have the same absolute probability. The values in this table were derived using equations [5.2.2.2] and [5.2.4.2] presented in Chapter 5.

In our first experiment we analyzed the mazes when the compute time for all nodes was a constant 750. We performed the experiment twice; once with dominant probabilities and once with equal visit probabilities. Tables 7.1 summarizes the results of the experiment.

In the case of equal visit probabilities both heuristics achieve results that are consistently close to he expected speedup values listed in Table 7.0. In the case where a dominant path is present we see that the speedup factors realized are less than the theoretical values. In the case of dominant paths since the potential speedup is so large the results are more sensitive to scheduling overheads than in the case where dominant paths are not present. When there are no dominant paths present the actual speedups anticipated are so low, that even if a moderate amount of processing time is "lost" to scheduling the eager computation of rooms only a small percentage of the "lost" would have been put to productive use. When dominant paths are present a larger amount of the wasted time would have had the potential to be productive time. 
Table 7.1 - Comparison of Heuristics On Dialog Graphs With

Equal Node Probabilities Constant Compute Times of 750

Select Most Probable Nodes

Equal Node Probabilities

Select Most Probable Path

\begin{tabular}{|c|c|c|c|c|c|c|c|c|c|}
\hline \multicolumn{4}{|c|}{ Select Most Probable Path } & \multicolumn{3}{|c|}{ Speculative Version } & \multicolumn{3}{|c|}{ Mandatory Version } \\
\hline Doors & Procs & $\begin{array}{l}\text { Avg } \\
\text { Soquent- } \\
\text { ial Time }\end{array}$ & $\begin{array}{l}90 \% \\
C . I . \\
(+1-)\end{array}$ & $\begin{array}{l}\text { Average } \\
\text { Exoc } \\
\text { Time }\end{array}$ & $\begin{array}{l}90 \% \\
C .1 . \\
(+1-)\end{array}$ & $\begin{array}{l}A v_{i 3} \\
\text { Spexd } \\
\text { Uin }\end{array}$ & $\begin{array}{l}\text { Average } \\
\text { Exec } \\
\text { Time }\end{array}$ & $\begin{array}{l}90 \% \\
\text { C.1. } \\
(+1-)\end{array}$ & $\begin{array}{l}\text { Avg } \\
\text { Speod } \\
\text { U }\end{array}$ \\
\hline 2 & $\begin{array}{l}2 \\
4 \\
8\end{array}$ & $\begin{array}{l}158000 \\
315000 \\
631000\end{array}$ & $\begin{array}{l}0 \\
0 \\
0\end{array}$ & $\begin{array}{l}116000 \\
186000 \\
330000\end{array}$ & $\begin{array}{l}22100 \\
47200 \\
61700\end{array}$ & $\begin{array}{l}1.4 \\
1.7 \\
1.9\end{array}$ & $\begin{array}{l}130000 \\
208000 \\
372000\end{array}$ & $\begin{array}{l}29900 \\
47000 \\
66900\end{array}$ & $\begin{array}{l}1.2 \\
1.5 \\
1.7\end{array}$ \\
\hline 3 & $\begin{array}{l}2 \\
4 \\
8\end{array}$ & $\begin{array}{l}158000 \\
315000 \\
631000\end{array}$ & $\begin{array}{l}0 \\
0 \\
0\end{array}$ & $\begin{array}{l}135000 \\
240000 \\
429000\end{array}$ & $\begin{array}{l}30200 \\
56200 \\
49600\end{array}$ & $\begin{array}{l}1.2 \\
1.3 \\
1.5\end{array}$ & $\begin{array}{l}147000 \\
260000 \\
476000\end{array}$ & $\begin{array}{l}29800 \\
46200 \\
47200\end{array}$ & $\begin{array}{l}1.1 \\
1.2 \\
1.3\end{array}$ \\
\hline 4 & $\begin{array}{l}2 \\
4 \\
8\end{array}$ & $\begin{array}{l}158000 \\
315000 \\
631000\end{array}$ & $\begin{array}{l}0 \\
0\end{array}$ & $\begin{array}{l}147000 \\
258000 \\
444000 \\
\end{array}$ & $\begin{array}{l}23800 \\
39800 \\
41200 \\
\end{array}$ & $\begin{array}{l}1.1 \\
1.2\end{array}$ & $\begin{array}{l}155000 \\
280000 \\
518000\end{array}$ & $\begin{array}{l}25500 \\
31500 \\
49900\end{array}$ & $\begin{array}{l}1.0 \\
1.1 \\
1.2\end{array}$ \\
\hline
\end{tabular}

Dominant Path Present

\begin{tabular}{|c|c|c|c|c|c|c|c|c|c|}
\hline \multicolumn{4}{|c|}{ Select Most Probable Path } & \multicolumn{3}{|c|}{ Speculative Version } & \multicolumn{3}{|c|}{ Mandalory Version } \\
\hline Doors & Procs & $\begin{array}{c}\text { Avg } \\
\text { Sequent- } \\
\text { jal Time }\end{array}$ & $\begin{array}{l}90 \% \\
\text { C.I. } \\
(+1-)\end{array}$ & $\begin{array}{l}\text { Average } \\
\text { Exec } \\
\text { Time }\end{array}$ & $\begin{array}{l}90 \% \\
C 1 . \\
(+1-)\end{array}$ & $\begin{array}{l}\text { Avg } \\
\text { Spexd } \\
\text { Up }\end{array}$ & $\begin{array}{l}\text { Average } \\
\text { Fxoc } \\
\text { Time }\end{array}$ & $\begin{array}{l}\mathbf{C}) \% \\
C .1 . \\
(+1-)\end{array}$ & $\begin{array}{l}\text { Avg } \\
\text { Speod } \\
\text { Up }\end{array}$ \\
\hline 2 & $\begin{array}{l}2 \\
4 \\
8\end{array}$ & $\begin{array}{l}158000 \\
315000 \\
631000\end{array}$ & $\begin{array}{l}0 \\
0 \\
0\end{array}$ & $\begin{array}{r}88300 \\
106000 \\
130000\end{array}$ & $\begin{array}{l}11200 \\
33000 \\
33000\end{array}$ & $\begin{array}{l}1.8 \\
3.0 \\
4.8\end{array}$ & $\begin{array}{r}95600 \\
117000 \\
145000\end{array}$ & $\begin{array}{l}18300 \\
42500 \\
38200\end{array}$ & $\begin{array}{l}1.7 \\
2.7 \\
4.3\end{array}$ \\
\hline 3 & $\begin{array}{l}2 \\
4 \\
8\end{array}$ & $\begin{array}{l}158000 \\
315000 \\
631000 \\
\end{array}$ & $\begin{array}{l}\overline{0} \\
0 \\
0\end{array}$ & $\begin{array}{r}89100 \\
106000 \\
133000 \\
\end{array}$ & $\begin{array}{l}11100 \\
33300 \\
31400\end{array}$ & $\begin{array}{l}1.8 \\
3.0 \\
4.7\end{array}$ & $\begin{array}{r}91100 \\
116000 \\
1460000\end{array}$ & $\begin{array}{l}14600 \\
40200 \\
43800\end{array}$ & $\begin{array}{l}1.7 \\
2.7 \\
4.3\end{array}$ \\
\hline 4 & $\begin{array}{l}2 \\
4\end{array}$ & $\begin{array}{l}158000 \\
315000 \\
631000\end{array}$ & $\begin{array}{l}0 \\
0\end{array}$ & $\begin{array}{r}89800 \\
112000 \\
138000\end{array}$ & $\begin{array}{l}10500 \\
33700 \\
27200\end{array}$ & $\begin{array}{l}1.8 \\
2.8 \\
4.6\end{array}$ & $\begin{array}{l}104000 \\
127000 \\
159000\end{array}$ & $\begin{array}{l}17500 \\
44100 \\
35600\end{array}$ & $\begin{array}{l}1.5 \\
2.5 \\
4.0\end{array}$ \\
\hline
\end{tabular}

Each average is compute based on the results of selecting 10 random paths through the same graph. The same graph and compute times are used for each of the 10 paths. In the case of a dominant path, each node has one descendaut that will be visited with $90 \%$ probability. The remaining $10 \%$ is divided equally amongst the other descendants. 
Since the compute times are constant for all rooms, we would expect that the speedup for the mandatory and speculative version would be very similar. We can see that there is actually quite a large discrepancy between the speedups of these two approaches. The discrepancy occurs because in the time from when the focus is computed until the next mom is entered, one or more of the eagerly computed rooms is completely constructed. At this point, the Execute-Request-Group procedure gets the next room from the old focus' request group (as far as the Execute-Request-Group procedure is concerned the old focus is still computing) and starts to construct the room. In the speculative version the construction of the "extra" room is halted. However, in the mandatory version the construction of the "mandatory" room is continued to completion. If the room is actually required this will not impact the execution time for the mandatory version (it will actually improve it). However, if the room is not required, the processor is kept busy performing the irrelevant computations of the "extra" room. When equal visit probabilities are present, the likelihood that the computations are irrelevant is increased, thus the average execution time for the mandatory version is also increased.

In the case where a dominant path is present the speedup figures are much more exciting. (We only show the results for the Select Most Probable Path heuristic since in the case of the dominant paths that we performed our experiments with, both heuristics produce the same results). Both the Select Most Probable Nodes and Select Most Probable Path heuristics produce large speedups. If we compare the speedups achieved to the expected values we can see that the gathered results also suffer from the overhead of scheduling. In all cases, the speculative version produces speedups that are larger than those produced by the mandatory version. When eight processors are used to construct the maze, we achieve specdups in excess of 4.5. Even in the cases where 2 processors are used, we consistently 
achieve speedups of approximately 1.75. This experiment shows how important the accurate assignment of probabilities to request on achieving large speedups. If we can identify those requests that the user is most likely to make, we can dramatically improve tix: effective utilization of our processing resources.

In our next set of experiments we extended our first experiment by allowing the compute times to vary uniformly between $\mathbf{5 0 0}$ and $\mathbf{1 0 0 0}$. We did this in order to assess the impact of compute time variability on speedup behavior. Tables 7.2 summarizes the results of our experiments.

In the case of dominant paths, when each node had the same compute time, we were assured that if the nodes on the dominant path were cagerly constructed and the user stayed on the path, he/she should be able to move $\mathbf{N}$ (where $\mathbf{N}$ is the number of rooms on the critical path that we have computed). With variable compute times, it is equally likely that the next room on the dominant path will or will not require additional construction. If the compute time of the focus is less than the compute time of a room in the request group, the room will not be totally constructed by the time the focus has be constructed. However, in the case where the compute time of the focus is greater than the compute time of a room in the request group, we can compute more than $\mathbf{N}$ rooms. This increases the chance the user will not have to wait at the next room they enter. 
Table 7.2 - Comparison of Heuristics On Dialog Graphs With Random Compute Times Between 500 and 1000

Hqual Node Probabilities

Select Mosi Probable Nodes

\begin{tabular}{|c|c|c|c|c|c|c|c|c|c|}
\hline \multirow{2}{*}{ Doors } & Procs & $\begin{array}{c}\text { Avg } \\
\text { Sequent- } \\
\text { ial Time }\end{array}$ & $\begin{array}{c}90 \% \\
\text { C.I. } \\
(+/)\end{array}$ & $\begin{array}{c}\text { Average } \\
\text { Exec } \\
\text { Time }\end{array}$ & $\begin{array}{c}90 \% \\
\text { C.I. } \\
(+/-)\end{array}$ & $\begin{array}{c}\text { Avg } \\
\text { Speed } \\
\text { Up }\end{array}$ & $\begin{array}{c}\text { Average } \\
\text { Exec } \\
\text { Time }\end{array}$ & $\begin{array}{c}90 \% \\
\text { C.I. } \\
(+/-)\end{array}$ & $\begin{array}{c}\text { Avg } \\
\text { Speod } \\
\text { Up }\end{array}$ \\
\hline 2 & 2 & 170000 & 15900 & 125000 & 27700 & 1.4 & 140000 & 22900 & 1.2 \\
& 4 & 331000 & 31400 & 171000 & 11300 & 1.9 & 215000 & 21600 & 1.5 \\
& 8 & 656000 & 38400 & 226000 & 15300 & 2.9 & 308000 & 18000 & 2.1 \\
\hline 3 & 2 & 170000 & 14900 & 133000 & 33500 & 1.3 & 156000 & 23900 & 1.1 \\
& 4 & 330000 & 20100 & 181000 & 23700 & 1.8 & 239000 & 19000 & 1.4 \\
& 8 & 643000 & 32700 & 266000 & 19300 & 2.4 & 374000 & 20400 & 1.7 \\
\hline 4 & 2 & 172000 & 17300 & 148000 & 25400 & 1.2 & 176000 & 21600 & 0.98 \\
& 4 & 336000 & 29900 & 204000 & 26200 & 1.6 & 255000 & 28500 & 1.3 \\
& 8 & 653000 & 36900 & 286000 & 23400 & 2.3 & 405000 & 37800 & 1.6 \\
\hline
\end{tabular}

Iiyual Node Probabilities

Seloct Most Probable Path

\begin{tabular}{|c|c|c|c|c|c|c|c|c|c|}
\hline Doors & Procs & $\begin{array}{l}\text { Avg } \\
\text { Sequemt- } \\
\text { ial Time }\end{array}$ & $\begin{array}{l}90 \% \\
\text { C.I. } \\
(+1-)\end{array}$ & $\begin{array}{l}\text { Average } \\
\text { Exoc } \\
\text { Time }\end{array}$ & $\begin{array}{l}90 \% \\
\text { C.1. } \\
(+1 .)\end{array}$ & $\begin{array}{l}\text { Avg } \\
\text { Speod } \\
\text { Up }\end{array}$ & $\begin{array}{l}\text { Avernge } \\
\text { Exec } \\
\text { Time }\end{array}$ & $\begin{array}{l}90 \% \\
\text { C.I. } \\
(+1-)\end{array}$ & $\begin{array}{l}\text { Avg } \\
\text { Speod } \\
\text { Up }\end{array}$ \\
\hline 2 & $\begin{array}{l}2 \\
4 \\
8\end{array}$ & $\begin{array}{l}170000 \\
330000 \\
656000\end{array}$ & $\begin{array}{l}15900 \\
31400 \\
38400\end{array}$ & $\begin{array}{l}136000 \\
216000 \\
361000\end{array}$ & $\begin{array}{r}36100 \\
65700 \\
101000 \\
\end{array}$ & $\begin{array}{l}1.3 \\
1.5 \\
1.8\end{array}$ & $\begin{array}{l}149000 \\
246000 \\
399000\end{array}$ & $\begin{array}{l}36000 \\
89100 \\
64400\end{array}$ & $\begin{array}{l}1.1 \\
1.3 \\
1.6\end{array}$ \\
\hline 3 & $\begin{array}{l}2 \\
4 \\
8\end{array}$ & $\begin{array}{l}170000 \\
330000 \\
643000\end{array}$ & $\begin{array}{l}14900 \\
20100 \\
32700\end{array}$ & $\begin{array}{l}142000 \\
228000 \\
446000\end{array}$ & $\begin{array}{l}29600 \\
70600 \\
80500\end{array}$ & $\begin{array}{l}1.2 \\
1.4 \\
1.4\end{array}$ & $\begin{array}{l}165000 \\
284000 \\
490000\end{array}$ & $\begin{array}{l}21800 \\
45600 \\
68700\end{array}$ & $\begin{array}{l}1.0 \\
1.2 \\
1.3\end{array}$ \\
\hline$\overline{4}$ & $\begin{array}{l}2 \\
4 \\
8\end{array}$ & $\begin{array}{l}172000 \\
336000 \\
653000\end{array}$ & $\begin{array}{l}17300 \\
29900 \\
36900\end{array}$ & $\begin{array}{l}151000 \\
272000 \\
440000\end{array}$ & $\begin{array}{l}26100 \\
35600 \\
45400\end{array}$ & $\begin{array}{l}1.1 \\
1.2 \\
1.5\end{array}$ & $\begin{array}{l}169000 \\
300000 \\
556000\end{array}$ & $\begin{array}{l}\mathbf{3 0 9 0 0} \\
\mathbf{5 5 2 0 0} \\
\mathbf{3 4 6 0 0}\end{array}$ & $\begin{array}{l}1.0 \\
1.1 \\
1.2\end{array}$ \\
\hline
\end{tabular}

Dominant Path Present

Select Mosi Prohable Path

\begin{tabular}{|c|c|c|c|c|c|c|c|c|c|}
\hline \multirow{2}{*}{ Doors } & Procs & $\begin{array}{c}\text { Avg } \\
\text { Sequent- } \\
\text { ivl Time }\end{array}$ & $\begin{array}{c}90 \% \\
\text { C.I. } \\
(+/-)\end{array}$ & $\begin{array}{c}\text { Average } \\
\text { Exox } \\
\text { Time }\end{array}$ & $\begin{array}{c}90 \% \\
\text { C.I. } \\
(+/-)\end{array}$ & $\begin{array}{c}\text { Avg } \\
\text { Speod } \\
\text { Up }\end{array}$ & $\begin{array}{c}\text { Average } \\
\text { Exoc } \\
\text { Time }\end{array}$ & $\begin{array}{c}90 \% \\
\text { C.I. } \\
(+/-)\end{array}$ & $\begin{array}{c}\text { Avg } \\
\text { Speed } \\
\text { Up }\end{array}$ \\
\hline 2 & 2 & 170000 & 10700 & 100000 & 14800 & 1.7 & 101000 & 24400 & 1.7 \\
& 4 & 332000 & 24000 & 133000 & 36800 & 2.5 & 138000 & 45200 & 2.4 \\
& 8 & 658000 & 51400 & 166000 & 34800 & 4.0 & 177000 & 56400 & 3.7 \\
\hline 3 & 2 & 171000 & 19000 & 98600 & 17100 & 1.7 & 111000 & 40600 & 1.5 \\
& 4 & 332000 & 27600 & 125000 & 27700 & 2.6 & 164000 & 30600 & 2.0 \\
& 8 & 646000 & 34900 & 145000 & 41900 & 4.5 & 144000 & 44400 & 4.5 \\
\hline 4 & 2 & 163000 & 12800 & 102000 & 14600 & 1.6 & 113000 & 40200 & 1.4 \\
& 4 & 328000 & 19500 & 127000 & 29000 & 2.6 & 152000 & 62700 & 2.2 \\
& 8 & 649000 & 33900 & 136000 & 32100 & 4.8 & 163000 & 52700 & 4.0 \\
\hline
\end{tabular}

liach average is compute based on the results of selecting 10 random paths through the same graph. The same graph and compute times are used for each of the 10 paths. In the case of a dominant path, each node has one descendant that will be visited with $90 \%$ probability. The remaining $10 \%$ is divided equally anongst the other descendants. 
In the case of equal visit probabilities, the change in the speedup numbers was not dramatic and in general there was little change in the exceution time variance. The negligible change in the speedup numbers was to be expected since the performance of neither heuristic was outstanding when the compute time was constant for all nodes. The introduction of variable compute times could do little to worsen the performance of the heuristics.

The next experiment that we performed was an extension of the previous experiment. We increased the variance in compute times by allowing the times to be uniformly distrihuted between 0 and 1500 . We wanted to see what effect the further increase in variance would have on the speedup results. We correctly assumed that the increased variance would have the same basic effect that it had in the previous experiments; marginal speedups would remain marginal and other speedups would be lessened. Tables 7.4 and 7.5 summarize the results of our experiments. The effect of increasing the variance of the compute times is obvious; the greater the variance the smaller the speedup experienced.

In addition to enforcing our conviction that increased variance in compute times decreased speedup potential, we also observed that as the variance increased, the speedup experienced using the request groups from the Select Most Probable Path heuristic deteriorated faster than the speedup from the Select Most Probable Nodes heuristic. This phenomenon is easily explainable when we consider how "extra" processors are allocated to construct rooms not on the dominant path. An "extra" processor exists when all of the rooms on the dominant path have been constructed and we still have a processor or processors to allocate. The Select Most Probable Path heuristic allocates "extra" processors to construct rooms at the end of the dominant path. The Select Most Probable Nodes heuristic uses the processors to construct rooms near the user's current room. So in the event that the user deviates from the dominant path, the Select Most Probable Nodes heuristic will probably 
Table 7.3 - Comparison of Heuristics On Dialog Graphs With Random Compute Times Between 0 and 1500

Equal Node Probabilities Select Most Probahle Nodes

\begin{tabular}{|c|c|c|c|c|c|c|c|c|c|}
\hline Doors & Procs & $\begin{array}{l}\text { Avg } \\
\text { Sequent- } \\
\text { ial Time }\end{array}$ & $\begin{array}{l}90 \% \\
\text { C.I. } \\
(+/-)\end{array}$ & $\begin{array}{l}\text { Avernge } \\
\text { Exoc } \\
\text { Time }\end{array}$ & $\begin{array}{l}90 \% \\
\text { C.I. } \\
(t+)\end{array}$ & $\begin{array}{l}\text { Avg } \\
\text { Speed } \\
\text { Up }\end{array}$ & $\begin{array}{l}\text { Avenge } \\
\text { Erec } \\
\text { Time }\end{array}$ & $\begin{array}{l}90 \% \\
\text { C.I. } \\
(+/ 6)\end{array}$ & $\begin{array}{l}\text { Avg } \\
\text { Speed } \\
\text { Up }\end{array}$ \\
\hline 2 & $\begin{array}{l}2 \\
4 \\
8\end{array}$ & $\begin{array}{l}179000 \\
350000 \\
679000 \\
\end{array}$ & $\begin{array}{l}33000 \\
51700 \\
92900\end{array}$ & $\begin{array}{l}129000 \\
180000 \\
257000\end{array}$ & $\begin{array}{l}34500 \\
29100 \\
32600\end{array}$ & $\begin{array}{l}1.4 \\
1.9 \\
2.6\end{array}$ & $\begin{array}{l}149000 \\
219000 \\
324000\end{array}$ & $\begin{array}{l}38800 \\
34900 \\
19300\end{array}$ & $\begin{array}{l}1.2 \\
1.6 \\
2.1\end{array}$ \\
\hline 3 & $\begin{array}{l}2 \\
4 \\
8\end{array}$ & $\begin{array}{l}177000 \\
351000 \\
664000\end{array}$ & $\begin{array}{l}25700 \\
45800 \\
75600\end{array}$ & $\begin{array}{l}141000 \\
210000 \\
302000\end{array}$ & $\begin{array}{l}46300 \\
33100 \\
50800\end{array}$ & $\begin{array}{l}1.2 \\
1.7 \\
2.2\end{array}$ & $\begin{array}{l}165000 \\
255000 \\
382000\end{array}$ & $\begin{array}{l}30700 \\
41500 \\
51900\end{array}$ & 1.1 \\
\hline 4 & $\begin{array}{l}2 \\
4 \\
8\end{array}$ & $\begin{array}{l}178000 \\
340000 \\
641000\end{array}$ & $\begin{array}{l}43400 \\
68700 \\
88900\end{array}$ & $\begin{array}{l}142000 \\
196000 \\
313000\end{array}$ & $\begin{array}{l}40000 \\
31400 \\
54200\end{array}$ & $\begin{array}{l}1.3 \\
1.7 \\
2.0\end{array}$ & $\begin{array}{l}157000 \\
274000 \\
448000\end{array}$ & $\begin{array}{l}46200 \\
34600 \\
39500\end{array}$ & $\begin{array}{l}1.1 \\
1.2\end{array}$ \\
\hline
\end{tabular}

Equal Node Probabilitics

Select Most Probable Path

\begin{tabular}{|c|c|c|c|c|c|c|c|c|c|}
\hline \multicolumn{4}{|c|}{ Select Most Probable Peth } & \multicolumn{3}{|c|}{ Speculative Version } & \multicolumn{3}{|c|}{ Mandeaxy Verion } \\
\hline Doors & Procs & $\begin{array}{c}\text { Avg } \\
\text { Sequeat- } \\
\text { ial Time }\end{array}$ & $\begin{array}{l}90 \% \\
\text { C.I. } \\
(+1-)\end{array}$ & $\begin{array}{l}\text { Averge } \\
\text { Erex } \\
\text { Time }\end{array}$ & $\begin{array}{l}90 \% \\
C .1 . \\
(+/-)\end{array}$ & $\begin{array}{c}\text { Avg } \\
\text { Speed } \\
\text { Up }\end{array}$ & $\begin{array}{l}\text { Avenge } \\
\text { Exec } \\
\text { Time }\end{array}$ & $\begin{array}{l}90 \% \\
\text { C.I. } \\
(+t-)\end{array}$ & $\begin{array}{c}\text { Avg } \\
\text { Spoed } \\
\text { Up }\end{array}$ \\
\hline 2 & $\begin{array}{l}2 \\
4 \\
8\end{array}$ & $\begin{array}{l}179000 \\
350000 \\
678000\end{array}$ & $\begin{array}{l}33000 \\
51700 \\
92900\end{array}$ & $\begin{array}{l}133000 \\
226000 \\
404000\end{array}$ & $\begin{array}{r}36900 \\
72800 \\
104000\end{array}$ & $\begin{array}{l}1.3 \\
1.5 \\
1.7\end{array}$ & $\begin{array}{l}154000 \\
244000 \\
462000\end{array}$ & $\begin{array}{l}54600 \\
89200 \\
91000\end{array}$ & $\begin{array}{l}1.2 \\
1.4 \\
1.5\end{array}$ \\
\hline 3 & $\begin{array}{l}2 \\
4 \\
8\end{array}$ & $\begin{array}{l}177000 \\
351000 \\
664000\end{array}$ & $\begin{array}{l}25700 \\
45800 \\
75600\end{array}$ & $\begin{array}{l}149000 \\
269000 \\
444000\end{array}$ & $\begin{array}{l}48700 \\
89800 \\
96200\end{array}$ & $\begin{array}{l}1.2 \\
1.3 \\
1.5\end{array}$ & $\begin{array}{l}162000 \\
289000 \\
547000\end{array}$ & $\begin{array}{r}49000 \\
75400 \\
144000\end{array}$ & $\begin{array}{l}1.1 \\
1.2 \\
1.2\end{array}$ \\
\hline 4 & $\begin{array}{l}2 \\
4 \\
8\end{array}$ & $\begin{array}{l}178000 \\
340000 \\
641000\end{array}$ & $\begin{array}{l}3400 \\
68700 \\
88900\end{array}$ & $\begin{array}{l}140000 \\
282000 \\
477000\end{array}$ & $\begin{array}{l}40200 \\
63500 \\
90800\end{array}$ & $\begin{array}{l}1.3 \\
1.2 \\
1.3\end{array}$ & $\begin{array}{l}191000 \\
296000 \\
605000\end{array}$ & $\begin{array}{r}21800 \\
73700 \\
115000\end{array}$ & $\begin{array}{r}0.93 \\
1.1 \\
1.1\end{array}$ \\
\hline
\end{tabular}

Dominant Path Present

Select Most Probable Path

\begin{tabular}{|c|c|c|c|c|c|c|c|c|c|}
\hline \multicolumn{4}{|c|}{ Select Mest } & \multicolumn{3}{|c|}{ Speculative Version } & \multicolumn{3}{|c|}{ Mendatory Version } \\
\hline Doors & Procs & $\begin{array}{l}\text { Avg } \\
\text { Sequem- } \\
\text { ial Time }\end{array}$ & $\begin{array}{l}90 \% \\
\text { C.I. } \\
(+/-)\end{array}$ & $\begin{array}{l}\text { Average } \\
\text { Exec } \\
\text { Time }\end{array}$ & $\begin{array}{l}90 \% \\
\text { C.L. } \\
(+t)\end{array}$ & $\begin{array}{l}\text { Avg } \\
\text { Speod } \\
\text { Up }\end{array}$ & $\begin{array}{l}\text { Avernge } \\
\text { Exoc } \\
\text { Time }\end{array}$ & $\begin{array}{l}90 \% \\
\text { C.I. } \\
(+/-)\end{array}$ & $\begin{array}{c}\text { Avg } \\
\text { Speed } \\
\text { Up }\end{array}$ \\
\hline 2 & $\begin{array}{l}2 \\
4 \\
8\end{array}$ & $\begin{array}{l}169000 \\
351000 \\
681000\end{array}$ & $\begin{array}{r}40400 \\
73700 \\
107000\end{array}$ & $\begin{array}{l}104000 \\
127000 \\
161000\end{array}$ & $\begin{array}{l}41900 \\
45500 \\
56900\end{array}$ & $\begin{array}{l}1.6 \\
2.8 \\
4.2\end{array}$ & $\begin{array}{r}93700 \\
158000 \\
196000\end{array}$ & $\begin{array}{r}39900 \\
60900 \\
100000\end{array}$ & $\begin{array}{l}1.8 \\
2.2 \\
3.5\end{array}$ \\
\hline 3 & $\begin{array}{l}2 \\
4 \\
8\end{array}$ & $\begin{array}{l}157000 \\
328000 \\
650000\end{array}$ & \begin{tabular}{r|}
34200 \\
87600 \\
120000
\end{tabular} & $\begin{array}{l}111000 \\
137000 \\
180000\end{array}$ & $\begin{array}{l}22900 \\
39100 \\
47800\end{array}$ & $\begin{array}{l}1.4 \\
2.4 \\
3.6\end{array}$ & $\begin{array}{l}116000 \\
165000 \\
196000\end{array}$ & & $\begin{array}{l}1.4 \\
2.0\end{array}$ \\
\hline 4 & $\begin{array}{l}2 \\
4 \\
8\end{array}$ & $\begin{array}{l}164000 \\
341000 \\
644000\end{array}$ & $\begin{array}{r}60400 \\
98300 \\
117000 \\
\end{array}$ & $\begin{array}{l}108000 \\
122000 \\
170000\end{array}$ & & & $\begin{array}{l}108000 \\
132000 \\
214000\end{array}$ & & $\begin{array}{l}1.5 \\
2.6\end{array}$ \\
\hline
\end{tabular}

Each average is compule based on the results of selecting 10 random paths through the same graph. The same graph and compute times are used for each of the 10 paths. In the case of a dominont path, each node bas one descendent that will be visited with $90 \%$ probability. The remaining $10 \%$ is divided equally anongst the other descendants. 
have performed some "eager" computation on the surrounding rooms. The eager computations the Select Most Probable Path heuristic performs using the "extra" processors will only be of benefit if the user does not deviate from the dominant path. $\therefore$ - variance in compute times increases we would expect the deterioration trends to continue.

In an effort to ascertain how the heuristics would perform in a situation where both the compute times and the visit probabilities were variable, we performed experiment on the mazes with random compute times between 500 and 1000 and used random arc probabilities (for each node, every arc emanating from it was assigned a probahility that was a multiple of 0.1 and was never less than 0.1 ). Table 7.4 summarizes the results of the experiments.

Not surprisingly, the results show that the speedup is better than we can get when we do not know anything about the user's behavior (i.e., equal visit probabilities) but the speedup is also significantly less that can be achieved if we are very familiar with the user's behavior (i.e., dominant path present). This experiment cicarly illustrates that any knowledge about the user's behavior can provide us with significant specdup improvement, even if the knowledge does not allow for a dominant path to be plotted through the dialog. 
Table 7.4 - Comparison of Heuristics On Dialog Graphs With Random Compute Times Between 500 and 1000 and Random Arc Probabilities

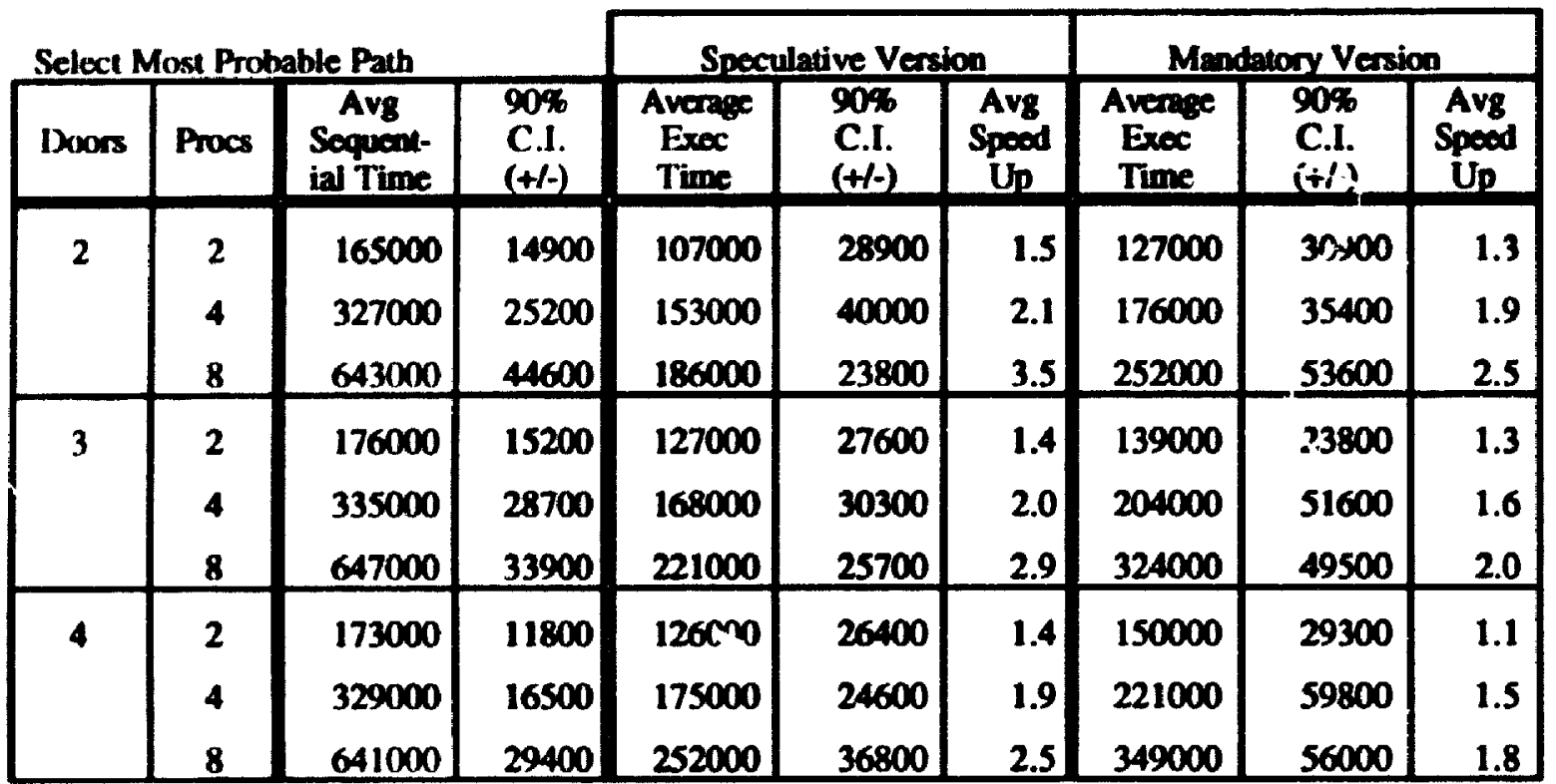

\begin{tabular}{|c|c|c|c|c|c|c|c|c|c|}
\hline \multicolumn{4}{|c|}{ Select Most Probable Nodes } & \multicolumn{3}{|c|}{ Speculative Version } & \multicolumn{3}{|c|}{ Mandalory Version } \\
\hline Dooss & Procs & $\begin{array}{l}\text { Avg } \\
\text { Sequent- } \\
\text { ial Time }\end{array}$ & $\begin{array}{l}90 \% \\
C . I . \\
(+/-)\end{array}$ & $\begin{array}{l}\text { Average } \\
\text { Exec } \\
\text { Time }\end{array}$ & $\begin{array}{l}90 \% \\
\text { C.I. } \\
(+/-)\end{array}$ & $\begin{array}{l}\text { Avg } \\
\text { Speed } \\
\text { Up }\end{array}$ & $\begin{array}{l}\text { Average } \\
\text { Exoc } \\
\text { Time }\end{array}$ & $\begin{array}{l}90 \% \\
\text { C.I. } \\
(+/-)\end{array}$ & $\begin{array}{l}\text { Avg } \\
\text { Speed } \\
\text { Up }\end{array}$ \\
\hline 2 & $\begin{array}{l}2 \\
4 \\
8\end{array}$ & $\begin{array}{l}165000 \\
326000 \\
643000 \\
\end{array}$ & $\begin{array}{l}14900 \\
25200 \\
44600 \\
\end{array}$ & $\begin{array}{l}111000 \\
144000 \\
205000 \\
\end{array}$ & $\begin{array}{l}22700 \\
64000 \\
63700 \\
\end{array}$ & $\begin{array}{l}1.5 \\
2.3 \\
3.1 \\
\end{array}$ & $\begin{array}{l}126000 \\
170000 \\
274000\end{array}$ & $\begin{array}{l}36200 \\
67800 \\
69000 \\
\end{array}$ & $\begin{array}{l}1.3 \\
1.9 \\
2.3 \\
\end{array}$ \\
\hline 3 & $\begin{array}{l}2 \\
4 \\
8 \\
\end{array}$ & $\begin{array}{l}176000 \\
335000 \\
647000 \\
\end{array}$ & $\begin{array}{l}15200 \\
28700 \\
33900 \\
\end{array}$ & $\begin{array}{l}122000 \\
196000 \\
304000 \\
\end{array}$ & $\begin{array}{r}24700 \\
48300 \\
61300 \\
\end{array}$ & $\begin{array}{l}1.4 \\
1.7 \\
2.1\end{array}$ & $\begin{array}{l}133000 \\
227000 \\
373000 \\
\end{array}$ & $\begin{array}{l}34600 \\
53100 \\
88100 \\
\end{array}$ & $\begin{array}{l}1.3 \\
1.5 \\
1.7 \\
\end{array}$ \\
\hline 4 & $\begin{array}{l}2 \\
4 \\
8\end{array}$ & $\begin{array}{l}173000 \\
329000 \\
641000 \\
\end{array}$ & $\begin{array}{l}11800 \\
16500 \\
29400 \\
\end{array}$ & $\begin{array}{l}126000 \\
192000 \\
302000\end{array}$ & $\begin{array}{l}22400 \\
59600 \\
44300\end{array}$ & $\begin{array}{l}1.4 \\
1.7 \\
2.1\end{array}$ & $\begin{array}{l}138000 \\
269000 \\
398000\end{array}$ & $\begin{array}{l}27100 \\
48600 \\
74900\end{array}$ & $\begin{array}{l}1.3 \\
1.2 \\
1.6\end{array}$ \\
\hline
\end{tabular}

Fach average is compute based on the results of selecting 10 random paths through the sane graph. The same graph and compute times are used for each of the 10 paths. 
As our previous experiments have shown, on the average there is not a large difference between the speedups achieved by the speculative and the mandatory versions. In the case of dominant paths we find that the average difference in the speedup created hy each version has a definite advantage over the mandatory version is when there are large variances in the compute times of the nodes relative to cach other. Since the mandatory version does not abort useless computation, if it should become involved in a lengthy computation that is found to be irrelevant, the computation of the room will tic up a processor for a considerable period. Since the speculative version aborts uscless computations it will not suffer the same type of processor loss, thus effectively having one more processor that the mandatory version. We can extend this scenario until all of the mandatory versions processors are busy performing useless computations while the speculative version's processors are busy doing possibly relevant work. Achieving this type of scenario in practice is rare since the user must always select rooms that have short compute times and avoid those rooms with long compute times. If the user selects a room with a long compute time, then the mandatory version will be able to finish all of its "irrelevant" computations and commence new, possibly relevant computations.

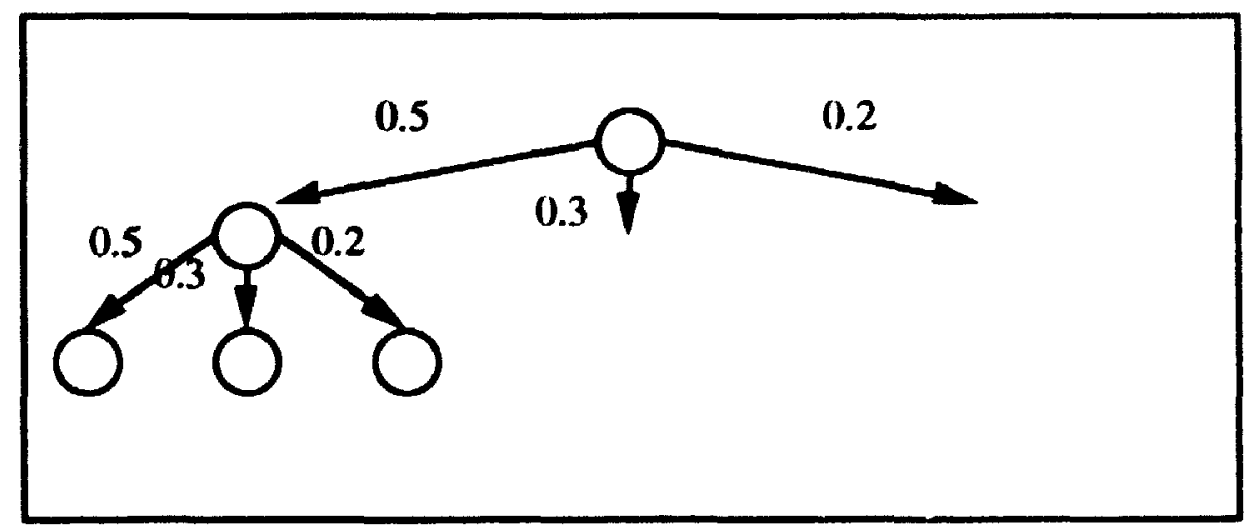

Figure 7.3 - Dialog Graph Structure 
Tahle 7.5 - Comparisor, of Heuristics On Dialog Graphs where Large Variation between Room Compute Times

\begin{tabular}{|c|c|c|c|c|c|c|c|c|c|}
\hline \multicolumn{4}{|c|}{ Selut Most Probahle Noxkes } & \multicolumn{3}{|c|}{ Speculative Version } & \multicolumn{3}{|c|}{ Mandatory Version } \\
\hline $\begin{array}{l}\text { Prob } \\
\text { Room } \\
\text { 8000 }\end{array}$ & Procs & $\begin{array}{l}\text { Avg } \\
\text { Sequent- } \\
\text { ial Time }\end{array}$ & $\begin{array}{l}90 \% \\
C .1 . \\
(+1-)\end{array}$ & $\begin{array}{l}\text { Average } \\
\text { Exec } \\
\text { Time }\end{array}$ & $\begin{array}{l}90 \% \\
\text { C.I. } \\
(+1 .)\end{array}$ & $\begin{array}{l}\text { Avg } \\
\text { Spoed } \\
\text { Up }\end{array}$ & $\begin{array}{l}\text { Average } \\
\text { Exec } \\
\text { Time }\end{array}$ & $\begin{array}{l}90 \% \\
\text { C.I. } \\
(+1-)\end{array}$ & $\begin{array}{c}\text { Avg } \\
\text { Spoed } \\
\text { Up }\end{array}$ \\
\hline $25 \%$ & $\begin{array}{l}2 \\
4 \\
8\end{array}$ & $\begin{array}{r}406000 \\
910000 \\
2020000\end{array}$ & $\begin{array}{r}311000 \\
391000 \\
7280 \times 0 \\
\end{array}$ & $\begin{array}{r}416000 \\
568000 \\
899000 \\
\end{array}$ & $\begin{array}{r}266000 \\
239000 \\
360000 \\
\end{array}$ & $\begin{array}{r}0.98 \\
1.6 \\
2.2 \\
\end{array}$ & $\begin{array}{r}429000 \\
730000 \\
1540000 \\
\end{array}$ & $\begin{array}{r}291000 \\
271000 \\
411000 \\
\end{array}$ & $\begin{array}{r}0.95 \\
1.2 \\
1.3 \\
\end{array}$ \\
\hline $35 \%$ & $\begin{array}{l}2 \\
4 \\
8\end{array}$ & $\begin{array}{r}504000 \\
1130000 \\
2410000\end{array}$ & $\begin{array}{r}384000 \\
627000 \\
785000 \\
\end{array}$ & $\begin{array}{l}472000 \\
818000 \\
1460000\end{array}$ & $\begin{array}{r}248000 \\
257000 \\
313000 \\
\end{array}$ & 1.4 & $\begin{array}{r}555000 \\
930000 \\
1550000\end{array}$ & $\begin{array}{l}411000 \\
274000 \\
327000\end{array}$ & $\begin{array}{r}0.91 \\
1.2 \\
1.6\end{array}$ \\
\hline
\end{tabular}

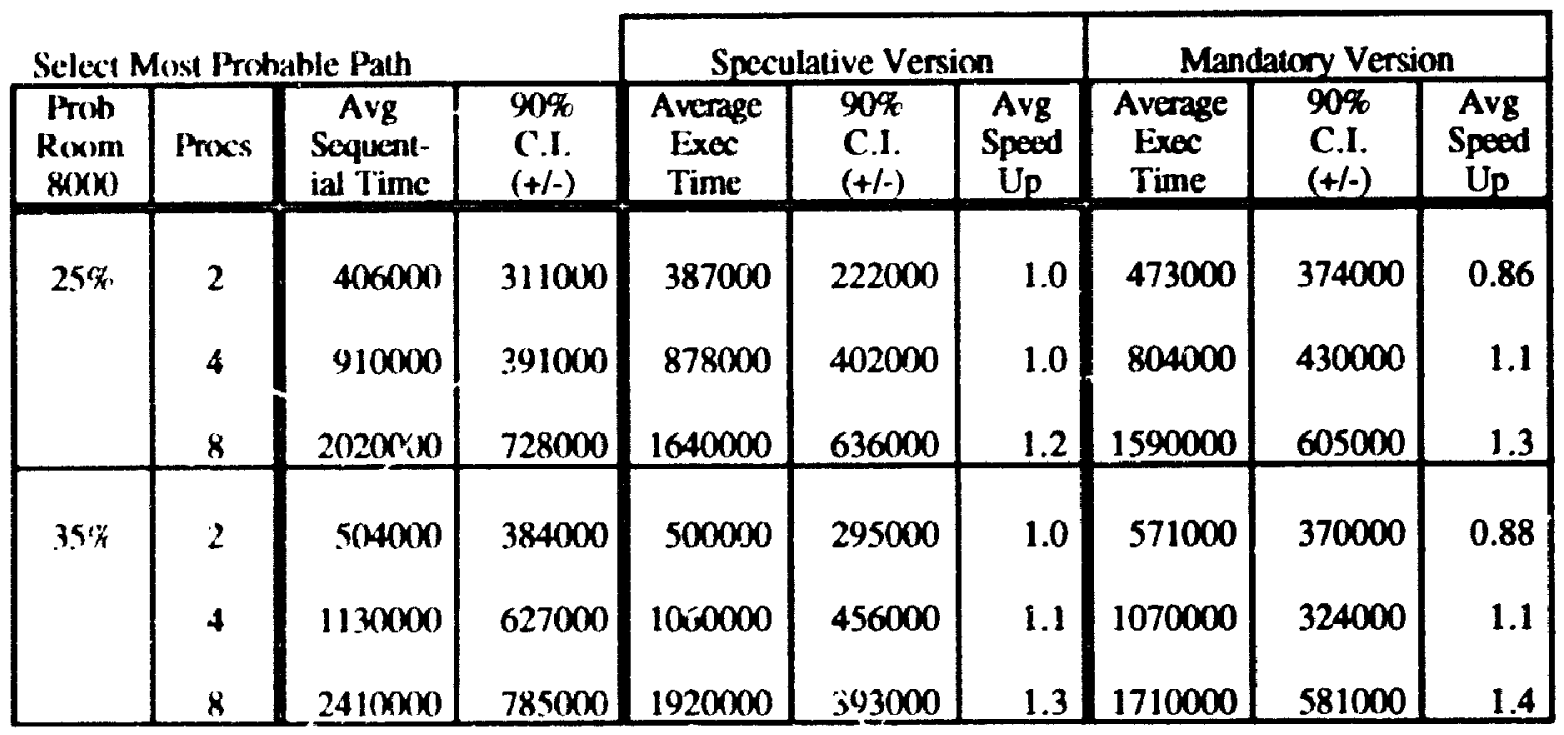


To gauge the effect that a high compute time variance might have on speedup, we have experimented with the interesting 3-ary graph shown in Figure 7.3. We chose two values for room compute times: 1000 units and 8000 . We performed the same set of experiments twice for different probabilities of a long or a short compute time room being encountered. Table 7.5 summarizes the results of our experiments. In our first experiment we used rooms with long compute times $35 \%$ ef the time. Since the probahilities assigned to the individual arcs do not create a dominant path, the version hased on the Select Most Probable Path heuristic performs poorly. More interesting is the Select Most Probable Nodes version. In the four processor and eight processors instances, the speedup of the sperulative version was 0.2 and 0.1 greater than the speedup of the mandatory versions respectively. When we changed the probability that a room with a long compute time was encountered to $25 \%$, the difference in the speedup figures increased dramatically. Experiments with $65 \%$ and $75 \%$ probabilities yiclded results with indicated that approximately the same speedup was achieved with the speculative and mandatory versions. From this experiment we can surmise that the speculative version will outperform the mandatory version by a sizable margin in those instances where a small portion of the rooms require a significant amount more computation than the other rexms.

In order to gauge the effect of think time on specdup, we performed a single set of experiments where the think times distribution was non-\%ero. We chose to perform our experiments on a graph where the compute time for each node was a constant 75) units and the think time was a constant 250 units. Table 7.6 summarizes the results of our experiments. As we can see from the results, the inclusion of non-zero think times increases the speedup potential quite dramatically. The largest difference between the speedup factors achieved by the speculative and mandatory is 2.1). This (xcurs when the Select Most Probable Nodes heuristic is employed on a graph where all nodes have equal 
Table 7.6 - Comparison of Heuristics On Dialog Graphs With Compute Times of 750 and Think Times of 250

I iqual Node Probabilities

\begin{tabular}{|c|c|c|c|c|c|c|c|c|c|}
\hline \multicolumn{4}{|c|}{ Selocl Most Prohable Nodes } & \multicolumn{3}{|c|}{ Speculative Version } & \multicolumn{3}{|c|}{ Mandatory Version } \\
\hline Ixors & Procs & $\begin{array}{l}\text { Avg } \\
\text { Sequent- } \\
\text { ial Time }\end{array}$ & $\begin{array}{l}90 \% \\
\text { C.I. } \\
(+1-)\end{array}$ & $\begin{array}{l}\text { Average } \\
\text { Exec } \\
\text { Time }\end{array}$ & $\begin{array}{l}90 \% \\
\text { C.I. } \\
(+1-)\end{array}$ & $\begin{array}{c}\text { Avg } \\
\text { Speed } \\
\text { Up }\end{array}$ & $\begin{array}{l}\text { Average } \\
\text { Exec } \\
\text { Time }\end{array}$ & $\begin{array}{l}90 \% \\
\text { C.I. } \\
(+/-)\end{array}$ & $\begin{array}{l}\text { Avg } \\
\text { Speed } \\
\text { Up }\end{array}$ \\
\hline 2 & $\begin{array}{l}2 \\
4 \\
8\end{array}$ & $\begin{array}{l}130000 \\
251000 \\
493000 \\
\end{array}$ & $\begin{array}{r}7150 \\
13100 \\
15100\end{array}$ & & & $\begin{array}{l}1.5 \\
2.5 \\
5.0\end{array}$ & & $\begin{array}{l}17500 \\
23900 \\
14700\end{array}$ & $\begin{array}{l}1.2 \\
1.7 \\
3.0\end{array}$ \\
\hline 3 & $\begin{array}{l}2 \\
4 \\
8\end{array}$ & $\begin{array}{l}132000 \\
254000 \\
501000\end{array}$ & $\begin{array}{r}6950 \\
12800 \\
14800\end{array}$ & $\begin{array}{l}113000 \\
126000 \\
169000\end{array}$ & $\begin{array}{r}27500 \\
8570 \\
34100 \\
\end{array}$ & $\begin{array}{l}1.2 \\
2.0 \\
3.0\end{array}$ & $\begin{array}{l}134000 \\
193000 \\
256000\end{array}$ & $\begin{array}{l}18600 \\
24400 \\
15500 \\
\end{array}$ & $\begin{array}{r}0.98 \\
1.3 \\
2.0 \\
\end{array}$ \\
\hline 4 & $\begin{array}{l}2 \\
4 \\
8\end{array}$ & $\begin{array}{l}134000 \\
260000 \\
511000\end{array}$ & $\begin{array}{r}6350 \\
12700 \\
11800\end{array}$ & $\begin{array}{l}130000 \\
150000 \\
205000\end{array}$ & $\begin{array}{l}22800 \\
20700 \\
63300\end{array}$ & $\begin{array}{l}1.0 \\
1.7 \\
2.5\end{array}$ & $\begin{array}{l}147000 \\
219000 \\
312000\end{array}$ & $\begin{array}{l}15700 \\
16600 \\
20000\end{array}$ & $\begin{array}{r}0.92 \\
1.2 \\
1.6\end{array}$ \\
\hline
\end{tabular}

liqual Node Probabilities

\begin{tabular}{|c|c|c|c|c|c|c|c|c|c|}
\hline \multicolumn{4}{|c|}{ Select Most Prohahle Path } & \multicolumn{3}{|c|}{ Speculative Version } & \multicolumn{3}{|c|}{ Mandatory Version } \\
\hline Doxis & I'rocs & $\begin{array}{c}\text { Avg } \\
\text { Sequent- } \\
\text { ial 'Time }\end{array}$ & $\begin{array}{l}90 \% \\
\text { C.I. } \\
(+/-)\end{array}$ & $\begin{array}{l}\text { Average } \\
\text { Exec } \\
\text { Time }\end{array}$ & $\begin{array}{l}90 \% \\
\text { C.I. } \\
(+1-)\end{array}$ & $\begin{array}{c}\text { Avg } \\
\text { Speod } \\
\text { Up }\end{array}$ & $\begin{array}{l}\text { Average } \\
\text { Exec } \\
\text { Time }\end{array}$ & $\begin{array}{l}90 \% \\
\text { C.I. } \\
(+/-)\end{array}$ & $\begin{array}{l}\text { Avg } \\
\text { Speed } \\
\text { Up }\end{array}$ \\
\hline 2 & $\begin{array}{l}2 \\
4 \\
8\end{array}$ & & $\begin{array}{l}7150 \\
13100 \\
15100\end{array}$ & & $\begin{array}{l}32200 \\
62600 \\
69300\end{array}$ & $\begin{array}{l}1.3 \\
1.4 \\
1.4\end{array}$ & & $\begin{array}{l}39500 \\
61400 \\
64200\end{array}$ & $\begin{array}{l}1.2 \\
1.3 \\
1.4\end{array}$ \\
\hline 3 & $\begin{array}{l}2 \\
4 \\
8\end{array}$ & $\begin{array}{l}132000 \\
254000 \\
501000\end{array}$ & $\begin{array}{r}6950 \\
12800 \\
14800\end{array}$ & $\begin{array}{l}128000 \\
237000 \\
455000\end{array}$ & $\begin{array}{l}38300 \\
59800 \\
54800\end{array}$ & $\begin{array}{l}1.0 \\
1.1 \\
1.1\end{array}$ & $\begin{array}{l}137000 \\
250000 \\
465000 \\
\end{array}$ & $\begin{array}{l}35600 \\
60600 \\
54200 \\
\end{array}$ & $\begin{array}{r}0.96 \\
1.0 \\
1.1 \\
\end{array}$ \\
\hline 4 & $\begin{array}{l}2 \\
4 \\
8\end{array}$ & $\begin{array}{l}134000 \\
260000 \\
511000 \\
\end{array}$ & $\begin{array}{r}6350 \\
12700 \\
11800 \\
\end{array}$ & $\begin{array}{l}143000 \\
263000 \\
498000\end{array}$ & $\begin{array}{l}32600 \\
42400 \\
48800\end{array}$ & $\begin{array}{r}0.94 \\
0.99 \\
1.0 \\
\end{array}$ & $\begin{array}{l}150000 \\
271000 \\
511000\end{array}$ & $\begin{array}{l}30400 \\
42400 \\
51100\end{array}$ & $\begin{array}{r}0.9 \\
0.96 \\
1.0\end{array}$ \\
\hline
\end{tabular}

Dominiant Path Present

\begin{tabular}{|c|c|c|c|c|c|c|c|c|c|}
\hline \multicolumn{4}{|c|}{ Sclect Most P'rohahle Path } & \multicolumn{3}{|c|}{ Speculative Version } & \multicolumn{3}{|c|}{ Mandatory Version } \\
\hline Denors & Procs & $\begin{array}{l}\text { Avg } \\
\text { Sequent- } \\
\text { ial lime }\end{array}$ & $\begin{array}{l}90 \% \\
\text { C.I. } \\
(+/-) \\
\end{array}$ & $\begin{array}{l}\text { Average } \\
\text { Exec } \\
\text { Time } \\
\end{array}$ & $\begin{array}{l}90 \% \\
\text { C.I. } \\
(+/-) \\
\end{array}$ & $\begin{array}{c}\text { Avg } \\
\text { Speod } \\
\text { Up }\end{array}$ & $\begin{array}{l}\text { Average } \\
\text { Exec } \\
\text { Time }\end{array}$ & $\begin{array}{l}90 \% \\
\text { C.I. } \\
(+1-) \\
\end{array}$ & $\begin{array}{l}\text { Avg } \\
\text { Speed } \\
\text { Up }\end{array}$ \\
\hline 2 & $\begin{array}{l}2 \\
4 \\
8\end{array}$ & $\begin{array}{l}130000 \\
251000 \\
493000\end{array}$ & $\begin{array}{r}7150 \\
13100 \\
15100\end{array}$ & $\begin{array}{l}62500 \\
62800 \\
96100\end{array}$ & $\begin{array}{l}18100 \\
45800 \\
52900\end{array}$ & $\begin{array}{l}2.1 \\
4.0 \\
5.1\end{array}$ & $\begin{array}{r}70200 \\
71700 \\
103000\end{array}$ & & $\begin{array}{l}1.9 \\
3.5 \\
4.8\end{array}$ \\
\hline 3 & $\begin{array}{l}2 \\
4 \\
8 \\
\end{array}$ & $\begin{array}{l}132000 \\
254000 \\
501000\end{array}$ & $\begin{array}{r}6950 \\
12800 \\
14800\end{array}$ & $\begin{array}{l}63400 \\
64800 \\
96300\end{array}$ & $\begin{array}{l}17900 \\
43300 \\
52200\end{array}$ & $\begin{array}{l}2.1 \\
3.9 \\
5.2\end{array}$ & $\begin{array}{r}72100 \\
71600 \\
103000\end{array}$ & $\begin{array}{l}26400 \\
50500 \\
53400\end{array}$ & $\begin{array}{l}1.8 \\
3.6 \\
4.9\end{array}$ \\
\hline 4 & $\begin{array}{l}2 \\
4 \\
8\end{array}$ & $\begin{array}{l}134000 \\
260000 \\
511000\end{array}$ & $\begin{array}{r}6350 \\
12 \% 00 \\
11800\end{array}$ & $\begin{array}{r}65700 \\
75700 \\
109000\end{array}$ & $\begin{array}{l}16500 \\
42800 \\
40700\end{array}$ & $\begin{array}{l}2.0 \\
3.4 \\
4.7\end{array}$ & $\begin{array}{r}77000 \\
83900 \\
111000\end{array}$ & $\begin{array}{l}24700 \\
57900 \\
43900\end{array}$ & $\begin{array}{l}1.7 \\
3.1 \\
4.6 \\
\end{array}$ \\
\hline
\end{tabular}

liach average is compute based on the results of selecting 10 random paths through the same graph. The same graph and compute times are used for each of the 10 paths. In the case of a dominant path, each nuke has one descendant that vill be visited with $90 \%$ probability. The remaining $10 \%$ is divided equally arnongst the other descendants. 
probabilities. If we compare the speedup difference for the same case ( 2 doors/roum, 8 processors) when a dominant path is present, we see that difference in specdup is down to 0.3. This once again illustrates the positive effect that a dominant path can have on increasing the likelihood that relevant computations are performed.

An interesting note about non-zero compute times that we discovered while deciding how long the think time should be is that the think time can nduce the speedup ratio to less than one. If we select the think time such that all of the adjacent rooms can be cagerly computed before the user stops thinking, the user will never have to wait for a room to be cumpuled (other than the initial room of course!). In these cases, adding additional proxessors will have no effect on the speedup factor achieved. Conversely, if we add sufficient prixessors to compute all of the adjacent rooms before the user stops thinking, the user will never have to wait.

In our final experiment we compared the Select Most Probable Nodes heuristic w the RGC algorithm. We performed our experiments using both equal visit probahilities and dominant paths. Our objective was to determine how close the heuristic comes to achieving the speedup of the algorithm. Tables 7.7 and 7.8 summarize the results of our experiments. As we can see, the Select Most Probable Nodes and the RCC: Algorithm achieve approximately the same speedup factors across all of the different test caxes. This show's that while the Select Most Probable Nodes heuristic is quite simple in technique in comparison to the RGC algorithm, the heuristic does an excellent jobs of allocating resources to keep the response time to a minimum. 
Table 7.7 - Comparison of RGC Algorithm and

Selcet Most Probable Nodes Heuristic On Dialog Graphs With

Compute Times of 1000 and Equal Visit Probabilities

\begin{tabular}{|c|c|c|c|c|c|c|c|c|c|}
\hline \multicolumn{4}{|c|}{ Rric Algorithm } & \multicolumn{3}{|c|}{ Speculative Version } & \multicolumn{3}{|c|}{ Mandatory Version } \\
\hline Dxours & Procs & $\begin{array}{c}\text { Avg } \\
\text { Sequent- } \\
\text { ial Time }\end{array}$ & $\begin{array}{l}90 \% \\
\text { C.I. } \\
(+1 .)\end{array}$ & $\begin{array}{l}\text { Average } \\
\text { Exec } \\
\text { Time }\end{array}$ & $\begin{array}{l}90 \% \\
\text { C.I. } \\
(+1 .)\end{array}$ & $\begin{array}{c}\text { Av8 } \\
\text { Spced } \\
\text { Up }\end{array}$ & $\begin{array}{l}\text { Average } \\
\text { Exoc } \\
\text { Time }\end{array}$ & $\begin{array}{l}90 \% \\
\text { C.I. } \\
(+1-)\end{array}$ & $\begin{array}{c}\text { Avg } \\
\text { Speed } \\
\text { Up }\end{array}$ \\
\hline \multirow[t]{3}{*}{2} & 2 & 189000 & 0 & 130000 & 24000 & 1.5 & 133000 & 23200 & 1.4 \\
\hline & 4 & 189000 & 0 & 92900 & 19600 & 2.0 & 94300 & 19700 & 2.0 \\
\hline & 8 & 189000 & 0 & 65500 & 1110 & 2.9 & 66200 & 270 & 2.9 \\
\hline \multirow[t]{3}{*}{3} & 2 & 147000 & 0 & 117000 & 28100 & 1.3 & 118000 & 27600 & 1.2 \\
\hline & 4 & 147000 & 0 & 85900 & 276 & 1.7 & 87200 & 297 & 1.7 \\
\hline & 8 & 147000 & 0 & 70700 & 14600 & 2.1 & 72000 & 14100 & 2.0 \\
\hline \multirow[t]{3}{*}{4} & 2 & 147000 & 0 & 134000 & 23100 & 1.1 & 135000 & 22800 & 1.1 \\
\hline & 4 & 147000 & 0 & 86100 & 723 & 1.7 & 87300 & 692 & 1.7 \\
\hline & 8 & 147000 & 0 & 78800 & 13300 & 1.9 & 80200 & 13900 & 1.8 \\
\hline
\end{tabular}

\begin{tabular}{|c|c|c|c|c|c|c|c|c|c|}
\hline \multicolumn{4}{|c|}{ Select Mont l'robable Nodes } & \multicolumn{3}{|c|}{ Speculative Version } & \multicolumn{3}{|c|}{ Mandatory Version } \\
\hline IXKors & Procs & $\begin{array}{l}\text { Avg } \\
\text { Sequent- } \\
\text { ial Time }\end{array}$ & $\begin{array}{l}90 \% \\
\text { C.I. } \\
(+/ .)\end{array}$ & $\begin{array}{l}\text { Average } \\
\text { Exoc } \\
\text { Time }\end{array}$ & $\begin{array}{l}90 \% \\
C .1 . \\
(+/-)\end{array}$ & $\begin{array}{c}\text { Avg } \\
\text { Speed } \\
\text { Up }\end{array}$ & $\begin{array}{c}\text { Average } \\
\text { Exec } \\
\text { Time }\end{array}$ & $\begin{array}{l}90 \% \\
\text { C.I. } \\
(+/-)\end{array}$ & $\begin{array}{c}\text { Avg } \\
\text { Spoed } \\
\text { Up }\end{array}$ \\
\hline \multirow[t]{3}{*}{2} & 2 & 189000 & 0 & 138000 & 37200 & 1.4 & 141000 & 35700 & 1.3 \\
\hline & 4 & 189000 & $\mathbf{0}$ & 93200 & 14300 & 2.0 & 94300 & 14000 & 2.0 \\
\hline & 8 & 189000 & 0 & 66100 & 680 & 2.9 & 66700 & 664 & 2.8 \\
\hline \multirow[t]{3}{*}{3} & 2 & 147000 & $\mathbf{0}$ & 119000 & 28400 & 1.2 & 120000 & 27700 & 1.2 \\
\hline & 4 & 147000 & $\mathbf{0}$ & 84200 & 8970 & 1.7 & 85600 & 9430 & 1.7 \\
\hline & 8 & 147000 & 0 & 70900 & 14400 & 2.1 & 71900 & 13700 & 2.0 \\
\hline \multirow[t]{3}{*}{4} & 2 & 147000 & $\mathbf{0}$ & 121000 & 31200 & 1.2 & 122000 & 30500 & 1.2 \\
\hline & 4 & 147000 & 0 & 92800 & 13800 & 1.6 & 93800 & 13900 & 1.6 \\
\hline & 8 & 147000 & 0 & 81700 & 12200 & 1.8 & 82200 & 12100 & 1.8 \\
\hline
\end{tabular}

liach average is compute hased on the results of selecting 10 random paths through the same graph. The sanc graph and compute times are used for each of the 10 paths. 
Table 7.8 - Comparison of RGC Algorithm and

Select Most Probable Nodes Heuristic On Dialog Graphs With

Compute Times of 1000 and Dominant Path Present

\begin{tabular}{|c|c|c|c|c|c|c|c|c|c|}
\hline \multicolumn{4}{|c|}{ Select Most Probable Nodes } & \multicolumn{3}{|c|}{ Speculative Version } & \multicolumn{3}{|c|}{ Manditory Version } \\
\hline Doors & Procs & $\begin{array}{l}\text { Avg } \\
\text { Sequent- } \\
\text { ial Time }\end{array}$ & $\begin{array}{l}90 \% \\
\text { C.I. } \\
(+1-)\end{array}$ & $\begin{array}{l}\text { Average } \\
\text { Exec } \\
\text { Time }\end{array}$ & $\begin{array}{l}90 \% \\
\text { C.I. } \\
(+1 .)\end{array}$ & $\begin{array}{c}\text { Avg } \\
\text { Spreol } \\
\text { Up }\end{array}$ & $\begin{array}{l}\text { Average } \\
\text { Exex } \\
\text { Time }\end{array}$ & $\begin{array}{l}40 \% \\
(.1 . \\
(+1-)\end{array}$ & $\begin{array}{l}\text { Avg } \\
\text { Spuxd } \\
\text { Up }\end{array}$ \\
\hline \multirow[t]{3}{*}{2} & 2 & 189000 & 0 & 108000 & 311 & 1.7 & 112000 & 440 & 1.7 \\
\hline & 4 & 189000 & 0 & 68900 & 12900 & 2.7 & 70300 & 12700 & 2.7 \\
\hline & 8 & 189000 & 0 & 50400 & 13800) & 3.8 & 52300 & $133(x)$ & 3.6 \\
\hline \multirow[t]{3}{*}{3} & 2 & 147000 & 0 & 86600 & 233 & 1.7 & $900 \times 0$ & 32 & 1.6 \\
\hline & 4 & 147000 & 0 & 52300 & 14400 & 2.8 & $531(x)$ & $151(0)$ & 2.8 \\
\hline & 8 & 147000 & 0 & 38100 & 19300 & 3.9 & $380 \times 00$ & 19? $(0)$ & 3.8 \\
\hline \multirow[t]{3}{*}{4} & 2 & 147000 & 0 & 86600 & 233 & 1.7 & $9 \times 000$ & 15 & 1.6 \\
\hline & 4 & 147000 & 0 & 54300 & 14600 & 2.7 & 5.5200 & $1.54(x)$ & 2.7 \\
\hline & 8 & 147000 & 0 & 40200 & 18200 & 3.7 & $4(9) \times(x)$ & $179 \times(x)$ & 3.6 \\
\hline
\end{tabular}

\begin{tabular}{|c|c|c|c|c|c|c|c|c|c|}
\hline \multicolumn{4}{|c|}{ RGC Algorithm } & \multicolumn{3}{|c|}{ Speculative Version } & \multicolumn{3}{|c|}{ Mandatury Version } \\
\hline Doors & Procs & $\begin{array}{l}\text { Avg } \\
\text { Sequent- } \\
\text { ial Time }\end{array}$ & $\begin{array}{l}90 \% \\
\text { C.I. } \\
(+1-)\end{array}$ & $\begin{array}{l}\text { Average } \\
\text { Exec } \\
\text { Time }\end{array}$ & $\begin{array}{l}90 \% \\
\text { C.I. } \\
(+/-)\end{array}$ & $\begin{array}{l}\text { Avg } \\
\text { Spood } \\
\text { Up }\end{array}$ & $\begin{array}{l}\text { Averape } \\
\text { lixex } \\
\text { Time }\end{array}$ & $\begin{array}{l}90)^{x} \\
(.1 . \\
(+1-)\end{array}$ & $\begin{array}{l}\text { Avg } \\
\text { Spuxd } \\
\text { Up }\end{array}$ \\
\hline \multirow[t]{3}{*}{2} & 2 & 189000 & 0 & 108000 & 311 & 1.7 & 112000 & 441) & 1.7 \\
\hline & 4 & 189000 & $\mathbf{0}$ & 70200 & 12200 & 2.7 & $707(x)$ & $127(x)$ & 2.7 \\
\hline & 8 & 189000 & 0 & 48500 & 12200 & 3.9 & $50 \times(x)$ & $122(x)$ & 3.8 \\
\hline \multirow[t]{3}{*}{3} & 2 & 147000 & 0 & 86600 & 233 & 1.7 & YXOXY) & 32 & 1.6 \\
\hline & 4 & 147000 & 0 & 52700 & 15000 & 2.8 & 53100 & $152(x)$ & 2.8 \\
\hline & 8 & 147000 & $\underline{0}$ & 38100 & $194(00)$ & 3.9 & $388(00)$ & $191(x)$ & 38 \\
\hline \multirow[t]{3}{*}{4} & 2 & 147000 & 0 & 86600 & 233 & 1.7 & $9 \times(\times \times)$ & 15 & 1.6 \\
\hline & 4 & 147000 & 0 & 54300 & 14600 & 2.7 & 55200 & $154(x)$ & 2.7 \\
\hline & 8 & 147000 & 0 & 40200 & 18200 & 3.7 & $4(x)(x)$ & $18(x)$ & 3.6 \\
\hline
\end{tabular}

Each average is compute based on the results of selecting 10 random paths through the same giant The same graph and compute times are used for each of the 10 paths. In each graph, each nocki has orie descendant that will be visited with $90 \%$ probability. Ibe remaining $10 \%$ is divided equally annongst the other descendants. 


\subsection{Comparison of Speculative and Mandatory Versions}

There is oflun a significant difference between the speedups produced by the mandatory and speculative versions of the game. This is especially svident when non-zero think times are present in graphs where there is no dominant path. In the cases where the think time is zero, we often find that the speculative version has speedups 0.5 greater than those if the mandatory version. In soine cases the difference is difference is almost 1.0 .

One experiment that we have not tried, but we feel would further illustrate the superiority of speculative computation over the use of mandatory computation would be to allow the user to make decisions based on partial results. Perhaps when a room is $25 \%$ or $50 \%$ constructed we would allow the user to decide what room to move to next, and make the move before the current room is completed. Since the mandatory version could not abort the ahandoned room, the user would have to wait for the room to be completed before hisher request could be honored. There are many applications that allow the user to make new requests before the current request has been completely computed. A number of areade type games allow the user to make moves before the entire screen has been displayed.

Aside from the increased speedup potential, speculative computation using Multilisp+SC offers a more flexible and robust approach to implementing parallel applications. Its support for creating, staying and coordinating tasks makes programming using speculative computation much simpler than programming using mandatory parallelism. It has been evident from programming the applications used for our experiments that programming 
with spe:ulative computation allows for a more natural expression of parallelism and an easier to understand program.

\subsection{Conclusions}

Table 7.9 summarizes the key results of our experiments. Both the Select Most Probahle Nodes and the Select Most Probable Path heuristics produce request groups that nesult in respectable speedup factors. In situations where the variance in compute time across nodes is high, the Select Most Probable Node heuristics produces superior results. If all nodes

\begin{tabular}{|c|c|}
\hline Experiment & Results \\
\hline Constant Compute Time & $\begin{array}{l}\text { Both speculative and mandatory implementations } \\
\text { achieve approximately the same results }\end{array}$ \\
\hline $\begin{array}{l}\text { Compute Times Between } \\
500 \text { and } 1000\end{array}$ & $\begin{array}{l}\text { Speculative implementation achieves speedup } 0.8 \\
\text { greater than mandatory version }\end{array}$ \\
\hline $\begin{array}{l}\text { Compute Times Between } \\
0 \text { and } 1500\end{array}$ & $\begin{array}{l}\text { Speculative implementation still achicves spexdup } \\
0.8 \text { greater than mandatory version }\end{array}$ \\
\hline $\begin{array}{l}\text { Compute Times Between } \\
500 \text { and } 1000 \text {, and } \\
\text { Random Arc Probabilities }\end{array}$ & $\begin{array}{l}\text { Both Select Most Probable Nodes and Select Most } \\
\text { Probable Path approaches produce favorable } \\
\text { Speedups. Speculative implementation achicves } \\
\text { speedup 1.0 greater than mandatory version }\end{array}$ \\
\hline $\begin{array}{l}\text { Large Variance Between } \\
\text { Compute Times }\end{array}$ & $\begin{array}{l}\text { Speculative version appears to outperform } \\
\text { mandatory version when a few nodes require } \\
\text { very large compute times }\end{array}$ \\
\hline Non-Zero Think Time & $\begin{array}{l}\text { When no dominant path is present, the } \\
\text { speculative version is decidedly superior to } \\
\text { the mandatory version. }\end{array}$ \\
\hline
\end{tabular}

Table 7.9 -Summary of Results of Experiments

have approximately the same absolute probability, the Select Most Probable Nodes heuristic is superior. If a dominant path exists through the dialog and the compute time 
variance is low, the Select Most Prubable Path heuristic produces the best results. We have found that the Select Most Probable Nodes heuristic compares favorably to the RGC algorithm and there is little difference between the speedups attained from using either method. 


\section{Chapter 8}

\section{Conclusions and Future Work}

\subsection{Conclusions}

The major contributions of this thesis are:

1. The identification of the requirements needed by a user interface to be able to exploit a speculative style of computation.

2. A model for user/interface dialogs and techniques that allow for the analysis of the dialog to determine how resources should be allocated to minimize the expected overall response time.

3. Experimental evidence of the potential of speculative computation to improve response time for a user/interface dialog.

We discuss each of these major contributions. 


\section{Requirements}

We have identified three major requirements that must be fulfilled by an application if it is to make effective us of speculative computation.

1. We must be able to predict which requests a user is likely to make.

2. We must be able to execute the user's requests in parallel and the performance gain made possible by doing so must more than offset the overhead of using speculative computation.

3. To make more productive use of processors, it is desirable to be able to estimate the compute time and/or think time associated with each request that the user can make. These estimates allow us to determine how the eager computation of requests should be scheduled so that we are always eagerly computing the most probable requests.

These requirements provide us with a quick and effective means of determining if an application may benefit from being programmed using speculative computation.

\section{Model For User Interface Analysis}

In order to be ahle to gain the maximum benefit from using speculative coinputation, we must have some means of determining how to best allocate resources to reduce response time. We have provided a model to represent a user/interface dialog. Using this model, we can analyze the user requests and their interrelationships to determine how to best allocate available resources to minimize the expected overall response time. 
The time required by the RGC (Chapter 5. Section 5.2) algorithm to analyze a user/interface dialog is prohibitive for dialogs which consist of more than a few requests. We have analyzed the RGC algorithm to determine how it decides to allocate resources and we used this knowledge to construct two heuristics that efficiently approximate the activities of the algorithm. We have analyzed each of the heuristics to provide simple closed formulas to determine the potential speedups hy eagerly computing requests. While these formulas onily apply to a very restricted subset of possible dialog graphs, they still provide us with a crude method of speedup estimation. This can allow us to gauge the effect that increasing or decreasing the number of available processors can have on performance.

\section{Experimental Evidence}

We have implemented an application that we have used to measure the response time of various dialogs and how the response times of these dialogs are affected hy the use of speculation. We were able to exploit the use of speculative computation with our implementation and to demonstrate its potential for improving response time. The potential of speculative computation becomes more evident when there is no single request that is much more likely than the others or some requests require excessive time to compute their result. If the time required to process a request varies greatly from request to request, aborting useless computations becomes more important. Also, if the think time associated with each node is long. suppon for speculative computation is not as critical since most requests will be computed well in advance of their request. 
We have successfully met our goals: we have investigated the potential of speculative computation for improving application response time and shown new cxamples for the use of speculative computation.

\subsection{Problems and Limitations}

In this section we discuss some negative aspects of our approach.

\section{Processor Allocation}

The current dialog model does not support the notion that different requests may require a differing number of processors. We have assumed that the processing of each request requires exactly the same number of processors. This was a conscious decision -. we wanted to simplify the model so that we could gain a more thorough understanding of the problem. However, in more complex dialogs, individual requests may be programmed using mandatory or speculative computation. With these applications we may not wish to constrain all requests to require the same number of processors. We will most probably want to allocate all available processors to the execution of the focus request and eagerly compute the results of the other requests using any processing resources that the focus task does not fully utilize. We will need to add support for request dependant processor requirements.

\section{Compute and Think Time Distributions}

Our current model supports discrete distributions. While we have utilized the discrete uniform distribution, we have not analytically or experimentally examined more elaborate discrete distributions. These types of distributions would be interesting to examine since 
they allow for the specification of increasingly complex distributions and are not as difficult to examine as continuous distributions.

The inclusion of efficient suppon for continuous distributions, while difficult to achicve. is necessary if more elaborate styles of user interface are to be analyad. While it is true that a continuous distribution could be loosely approximated by a discrete distrihution. the resulting explosion of nodes that would occur, would make the use of continuous distributions useless except for in the most trivial examples. The analysis techniquess and the current set of possible reductions and transformations must be extended to support continuous distributions.

\section{Request Probabilities}

The probability of a user making a specific request can vary over the course of the dialog. This can be due in part to the user's current location in the dialog and the number of times that the user has already made the request. Our current model does not support the notion of time variant probabilities. To better assimilate the needs of the user in a possibly dynamic environment, support should be added for time variant probabilitics.

\subsection{Future Work}

\subsubsection{Theory of User/Interface Dialogs}

We need to further understand how a user interacts with an applications and how the user's time is spent during the course of the dialog. By improving our ability to predict 
the requests that the user will nuake, either by constructing the application so that the order of requests is well defined or by observing users and how they make decisions, we will be able to better focus resources so that the response tine can kept minimal.

We have assumed the assignment of request probabilities solely on the basis of observation. A more flexible and accurate means to assign probabilities would be to have the application reme :ber previous conversations that it has had with the user and to adapt the probabilitics associated with each request that it supports, based on the user's actions in past conversations. As the user makes requests of the application, the application could keep track of the frequency of specific requests, the order in which they are made, and the think and compute times required. By keeping either an installation or user-specific knowledge-base of these facts, the application could periodically reconfigure the parameters that it uses to decide what request to eagerly compute. This would he useful in an application which has a gradual learning curve. As the user becomes more and more familiar with the application and becomes more and more focused in hisher interactions, the application would adjust the eager computations so that it performs to better benefit the user.

\subsubsection{User Interface Applications}

While we have demonstrated the potential of speculative computation using a small application, the true test will come when larger applications are programmed using speculative computation. Applications developed in areas such as Virtual Reality and Muli-Media Hyper-Texl are among the most promising prospects for being programmed using speculative computation. These types of applications tend to have user requests 
that are well defined and will probably be among the first type's of user interface to be implemented on the next generation of affordable parallel computer, due to their processing requirements. 


\section{Appendix}

Algorithm Computing the E[ORT] of a Dialog Graph.

Variahles:

EORT.

Initialization:

Iniualize EORT to zaro.

Procedurc COMPUTE_EORT

Parms:

List of Node Time Allocations

Current Node

Boxdy:

11) Remove Node Time Allocation in Node Tim Allocations List for Current Node Insiance

|2| $\quad$ BORT :=EORT +

(* (Compute Time for Current Node - Node Time Allocation)

(Relative Probahility for Current Node))

13 For Each Descendant of Current Node

COMPUTE_EORT with 
List Of Node Time Allocations := List Of Node Time Alloxations

Augmented with Time Allowations

Contributed by the Curn'nt Node.

Activation:

COMPUTE_EORT with

List of Node Allocations := nil.

Current Node := Initial Focus.

NOTE:

1. The Node Time Allocations contibuted by a node is the list of the nodes in the node's request group and their associated processing times.

2. If there is no node allocation in the List of Node Allocations then the allocalion associated with that node is 0 . 


\section{References}

[Baker74] Kenneth R. Baker

Introduction to Sequencing and Scheduling

John Wilcy \& Sons, Inc., New York, 1974

[Batter] Batter, J. and Brooks F.

"GROPE-1"

IFIP Proceedings 7I, 1972, P. 759.

|Bellman57| Bellman, Richard

Dynamic Programming

Princeton University Press, New Jersey, 1957

|Bcliman62| Bellmar, Richard and Dre:fus, Stuart E.

Appliced Dynamic Programming

Princeton University Press, New Jerscy, 1962

[Bruno76] J.L. Bruno, E.G. Coffman, Jr., R.L. Graham, W.H. Kohler,

R. Sethi, K. Steiglitz, and J.D.Ullman

Computer and Job-Shop Scheduling Theory

John Wilcy \& Sons, Inc., New York, 1976 
[Bubenik] Bubenik, R. and Zwaencpoel, W.

Optimistic Make

IEEE Transactions on Computers, Fcb 1992. pp. 207 - 217

[Burton] F.W. Burton,

Speculative Computation, Parallelism and Functional Programming

IEEE Transactions on Computers, Dec 1985, pp. $119(1)-119.3$

[Dybvig] Dybvig, R.K.

The Scheme Programming Language

Prentice-Hall, Inc., Englewood Cliffs, New Jersey, 1987.

pp. 83-90

[Faconti] Faconti, Giorgio P. and Paterno, Fabic,

An Approach to the Formal Specification of the Components

of an Interaction

EUROGRAPHICS '90, Eurographics Ass(ciation, 199(), p. 481 49.3

[Feyok77] S. Feyok

"Transition Diagram Based CAV/HELP Systems"

International Journal of Man-Macine Studies, Vol. 9, 1977 pp. 3(x)-413

[Fuchs] Fuchs, H., Poulton, J., Eyles, J., and Greer T.

"Course-Grain and Fine Grain Parallelism in the Next Generation I'ixcl|

Planes Graphics System,"

Proceedings of the Intemational Conference and Exhibition on Parallel

Processing for Computer Vision and Display, Ncw

York: Springer-Verlag, 1988.

[Garey] Garey, M.R. and Johnson, D.S.

Computers and Intractability $A$ Guide to the Theory of NP.

Completeness

Bell Telephone Laboratories, Inc., 1979, pp. 124-127 
[Gupta75] James P. Ignizio and Jatinder N. D. Gupta

Operations Research in Decision Making

Crane, Russak \& Company, Inc., New York, 1975

[Halstead] Halstead, R.

Multilisp: A Language for Concurnent Symbolic Computation

ACM Trans. on Prog. Languages and Systems, October 1985, pp. 501-538

|Haynes| Haynes, C.T. and Friedman, D.P.

"Abstracting Time Preemptions With Engines"

Journal of Computer Languages

[Jacob85] Robert J. K. Jacob

"A State Transition Diagram Language for Visual Programming" IEEE, August 1985, pp. 51-59

[Karp66] Richard M. Karp. and Raymond E. Miller

"Properties of a Model for Parallel Computations: Determinacy,

Termination, Queucing

SIAM J. Appl. Math., Vol 14, No 6, pp. 1390-1411

[Kernighan] Kernighan, B.W. and Pike, R.

The UNIX Programming Environment

Prentice Hall, 1984, p. 241

[Miller] Miller, J.

Multischeme: A Parallel Processing System Based on MIT Scheme

TR-402, Laboratory for Computer Science, M.I.T., Sept. 1987

|Molloyl Molloy, M.

Fundamentals of Performance Modelling

Macmillan Publishing Company, New York, 1989, pp. 117-149 
[Osborne] Osborne, R.

Speculative Computation in Miitilisp

Ph. D. Thesis, EECS, M.I.T., November 1989

[Rheingold] Rheingold, Howard

Virtual Reality

Summit Books, New York, New York, 1991

[Suttner] Suttner, C. B.

Competition versus Cooperation

IJCAI'91 Work'hop. 1991

[Wagner69] Harvey M. Wagner

Principles of Operations Research

Prentice-Hall, Ic., New Jersey, 1969

[Witte] Witte, W., Ciramberlain, R., and Franklin, M.

Parallel Simulated Annealing Using Spoculative Computation

IEEE Trans. on Parallel and Dist. Comp. Systems, (xt. 1991, pp. 4x3-493 

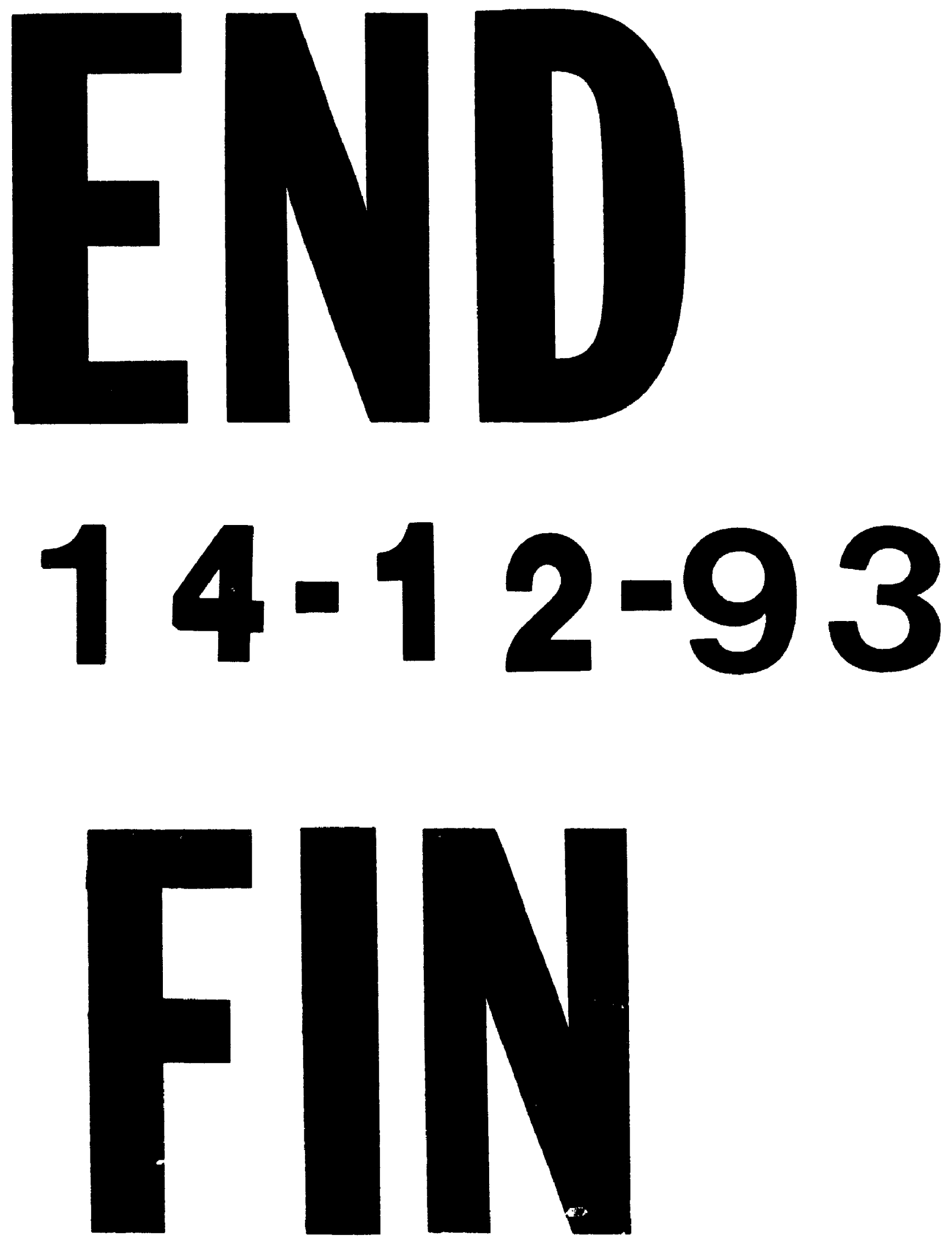